\title{
Adult trkB Signaling in Parvalbumin Interneurons is Essential to Prefrontal Network Dynamics
}

\author{
${ }^{\circledR}$ Nicolas Guyon, ${ }^{1}{ }^{\circledR}$ Leonardo Rakauskas Zacharias, ${ }^{2}{ }^{\circledR}$ Josina Anna van Lunteren, ${ }^{3}$ Jana Immenschuh, ${ }^{1}$

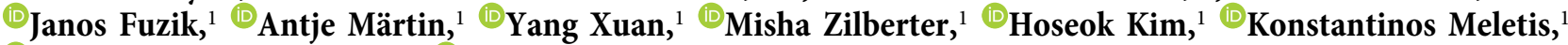 \\ ${ }^{\circledR}$ Cleiton Lopes-Aguiar, ${ }^{4}$ and ${ }^{\circledR}$ Marie Carlén ${ }^{1,3}$ \\ ${ }^{1}$ Department of Neuroscience, Karolinska Institutet, Stockholm 17177, Sweden, ${ }^{2}$ Department of Neuroscience and Behavioral Sciences, Ribeirão \\ Preto Medical School, Universidade de São Paulo, Ribeirão Preto 14049-900, Brazil, ${ }^{3}$ Department of Biosciences and Nutrition, Karolinska \\ Institutet, Huddinge 14183, Sweden, and ${ }^{4}$ Department of Physiology and Biophysics, Institute of Biological Sciences, Universidade Federal de Minas \\ Gerais, Belo Horizonte 31270-901, Brazil
}

Inhibitory interneurons expressing parvalbumin (PV) are central to cortical network dynamics, generation of $\gamma$ oscillations, and cognition. Dysfunction of PV interneurons disrupts cortical information processing and cognitive behavior. Brain-derived neurotrophic factor (BDNF)/tyrosine receptor kinase B (trkB) signaling regulates the maturation of cortical PV interneurons but is also implicated in their adult multidimensional functions. Using a novel viral strategy for cell-type-specific and spatially restricted expression of a dominant-negative trkB (trkB.DN), we show that BDNF/trkB signaling is essential to the integrity and maintenance of prefrontal PV interneurons in adult male and female mice. Reduced BDNF/trkB signaling in PV interneurons in the medial prefrontal cortex (mPFC) resulted in deficient PV inhibition and increased baseline local field potential (LFP) activity in a broad frequency band. The altered network activity was particularly pronounced during increased activation of the prefrontal network and was associated with changed dynamics of local excitatory neurons, as well as decreased modulation of the LFP, abnormalities that appeared to generalize across stimuli and brain states. In addition, our findings link reduced BDNF/trkB signaling in prefrontal $\mathrm{PV}$ interneurons to increased aggression. Together our investigations demonstrate that BDNF/trkB signaling in PV interneurons in the adult mPFC is essential to local network dynamics and cognitive behavior. Our data provide direct support for the suggested association between decreased trkB signaling, deficient PV inhibition, and altered prefrontal circuitry.

Key words: cortical inhibition; dominant-negative receptor; $\gamma$ oscillations; medial prefrontal cortex; neurotrophins; social behavior

\section{Significance Statement}

Brain-derived neurotrophic factor (BDNF)/tyrosine receptor kinase B (trkB) signaling promotes the maturation of inhibitory parvalbumin (PV) interneurons, neurons central to local cortical dynamics, $\gamma$ rhythms, and cognition. Here, we used a novel viral approach for reduced BDNF/trkB signaling in PV interneurons in the medial prefrontal cortex (mPFC) to establish the role of BDNF/trkB signaling in adult prefrontal network activities. Reduced BDNF/trkB signaling caused pronounced morphologic alterations, reduced PV inhibition, and deficient prefrontal network dynamics. The altered network activity appeared to manifest across stimuli and brain states and was associated with aberrant local field potential (LFP) activities and increased aggression. The results demonstrate that adult BDNF/trkB signaling is essential to PV inhibition and prefrontal circuit function and directly links BDNF/trkB signaling to network integrity in the adult brain.

Received July 16, 2020; revised Jan. 16, 2021; accepted Jan. 25, 2021.

Author contributions: N.G., L.R.Z., J.A.v.L., J.F., Y.X., K.M., C.L.-A., and M.C. designed research; N.G., L. R.Z., J.A.v.L., J.I., A.M., Y.X., M.Z., and C.L.-A. performed research; K.M. contributed unpublished reagents/analytic tools; N.G., L.R.Z., J.A.v.L., J.I., J.F., A.M., M.Z., H.K., and C.L.-A. analyzed data; N.G., L. R.Z., and M.C. wrote the paper.

This work was supported by a STINT Program Joint Brazilian-Swedish Research Collaboration grant BR2014-5869, Coordenação de Aperfeiçoamento de Pessoal de Nível Superior-Brasil (CAPES)- STINT Program Grant 99999.009883/2014-02, European Research Council Starting Grant 337069, and by the CAPES Finance Code 001. M.C. was supported by the Wallenberg Academy Fellow in Medicine Grant KAW 2012.0131 from the Knut and Alice Wallenberg Foundation, the Swedish Research Council Grant 2016-02700, and Karolinska Institutet Grant 2016-00139. C.L.-A. was supported by a São Paulo Research Foundation (FAPESP) Grant 2012/ 07107-2. Y.X., A.M., and J.A.v.L. were supported by doctoral grants from Karolinska Institutet (KID funding).
Part of cell imaging was performed in the Biomedicum Imaging Core (BIC) with support from the Karolinska Institutet. We thank Lino Tessarollo and Mary Ellen Palko for generously donating the pLTM570 plasmid; Maria Lindskog, André Fisahn, and Richard Andersson for advice on ex vivo electrophysiology experiments; Daniela Calvigioni for helping with the morphology analysis; Moritz Weglage for helping with the behavioral recording setup; and Cantin Ortiz for helping with the social interaction analysis custom-made script. Image of neurons in Figure $1 E$ and brain in Figure $1 F$ were adapted from Federico Claudi and Jiyun Shin, SciDraw (https://scidraw.io/).

The authors declare no competing financial interests.

Correspondence should be addressed to Marie Carlén at marie.carlen@ki.se.

https://doi.org/10.1523/JNEUROSCl.1848-20.2021

Copyright $\odot 2021$ the authors 


\section{Introduction}

Brain-derived neurotrophic factor (BDNF), the most widely expressed neurotrophin in the mammalian brain, mediates many activity-dependent processes and regulates the differentiation and growth of neurites, as well as the formation of synapses, during developmental refinement of circuit connectivity (for review, see Park and Poo, 2013). The maturation of parvalbumin (PV) interneurons and the cortical inhibition promoted by BDNF are integral to the onset of the critical period of cortical plasticity (Huang et al., 1999; for review, see Takesian and Hensch, 2013). Critical period closure breaks the plasticity and stabilizes the networks, and the PV interneurons thereafter maintain a high activity state (Reh et al., 2020). PV interneurons in the adult brain regulate the excitability and spike timing of local pyramidal neurons (PNs) through both feedback and feedforward mechanisms (for review, see Hu et al., 2014). PV interneuron mediated inhibition underlie the generation of local oscillatory network activity in the $\gamma$ range (30-90 Hz; Cardin et al., 2009; Buzsáki and Wang, 2012) and is also suggested to enable the formation of neuronal ensembles, including through suppression of less efficiently recruited neurons (Holtmaat and Caroni, 2016).

Both excitatory neurons and inhibitory interneurons in the cortex express tyrosine receptor kinase $\mathrm{B}$ (trkB), the high-affinity receptor for BDNF (Cellerino et al., 1996; Hashimoto et al., 2005), and BDNF binding to the full-length trkB activates several intracellular signaling pathways (Chao, 2003; Kowiański et al., 2018). C-terminal truncated trkB isoforms, lacking the catalytic tyrosine kinase domain, are also expressed in the brain and function as dominant-negative receptors, capable of inhibiting trkB signaling and BDNF function (Eide et al., 1996; Stoilov et al., 2002; Ohira and Hayashi, 2009). Much research on the role of $\mathrm{BDNF} / \mathrm{trkB}$ signaling in the mature nervous system has focused on plasticity processes changing the synaptic strength, particularly long-term potentiation (LTP). BDNF/trkB signaling also contributes to changes in intrinsic neuronal excitability in mature circuits by regulation of voltage-gated ion channels (Galloway et al., 2008; Park and Poo, 2013). However, little is known regarding the role of $\mathrm{BDNF} / \mathrm{trkB}$ signaling at the network level, particularly in the adult brain. Mouse models with genetic deletion of trkB in PV interneurons affect all trkB isoforms and impact postnatal maturation of PV neurons and brain circuitry dependent on BDNF/trkB signaling in large parts of the brain (Zheng et al., 2011; Tan et al., 2018; Xenos et al., 2018).

To circumvent these limitations, we here generated an adenoassociated viral (AAV) vector with Cre-dependent expression of a dominant-negative $\operatorname{trkB}(\operatorname{trkB} . \mathrm{DN})$ for direct investigation of how $\mathrm{BDNF} /$ trkB signaling contributes to the adult network functions of cortical PV interneurons. Targeting of trkB.DN to PV interneurons in the medial prefrontal cortex (mPFC) in adult mice and recording of single-units and local field potential (LFP) activity revealed that $\mathrm{BDNF} / \mathrm{trkB}$ signaling critically maintains the adult multidimensional function of cortical PV interneurons. Particularly, reduced BDNF/trkB signaling decreased the responsiveness of $\mathrm{mPFC} \mathrm{PV}$ interneurons ex vivo, manifesting as decreased spiking activity under conditions with increased drive. Detailed analysis of PV morphologies revealed marked neurite alterations, affecting both the dendrites and the axons. The in vivo experiments demonstrated increased aggression in mice with expression of trkB.DN in mPFC PV interneurons, characterized by increased recruitment of excitatory activity and failed induction of synchronous network activity over a broad frequency band. Importantly, the altered mPFC network dynamics appeared to generalize across stimuli and brain states. Our findings demonstrate the importance of intact $\mathrm{BDNF} / \mathrm{trkB}$ signaling in mature cortical networks, and couple changes in neurotrophic actions to structural and neurophysiological alterations in the adult brain.

\section{Materials and Methods}

\section{Animals}

All procedures were performed in accordance with the Guidelines of the Stockholm Municipal Committee for animal experiments (Stockholms djurförsöksetiska nämnd, approval N43/14). Animals used: Molecular and morphologic investigations: adult (8- to 16-week-old) male and female PV-Cre mice ( $n=12$; Jax stock \#008069). Ex vivo electrophysiology and morphology: adult (8- to 16-week-old) male and female PV-Cre $(n=41)$, Lhx6-eGFP mice ( $n=4$; used to visualize Lhx6 expressing interneurons in control experiments; MMRRC stock \#000246-MU) and Lhx6-eGFP/SST-Cre mice ( $n=1$; used to visualize Lhx6 expressing interneurons in control experiments; SST-Cre: Jax stock \#013044). In vivo electrophysiology and behavioral tests: adult (8- to 32-week-old) PV-Cre male mice $(n=36)$. For the resident-intruder procedure, male juvenile (three- to four-week-old) PV-Cre mice were used as intruders. Singlenucleus RNA sequencing (snRNA-seq): adult (nine-week-old) Vgat-Cre: H2B-eGFP male mice $(n=2$; see further below). Mice were housed in groups (two to five mice per cage) in individually-ventilated-cage systems (Sealsafe plus, GM 500, Tecniplast), under standardized conditions with a 12/12 h light/dark cycle (light 7:00 A.M.), stable temperature $\left(20 \pm 1^{\circ} \mathrm{C}\right)$ and humidity (40-50\%) with access to food (R70 Standard Diet, Lactamin AB; $4.5 \%$ fat, $14.5 \%$ protein, $60 \%$ carbohydrates) and water ad libitum. Implanted mice used for electrophysiology during behavior were single-housed after implantation $(n=10)$. All transgenic mice used were heterozygous for the transgene.

\section{Viral constructs and cre-dependent gene expression}

AAV-DIO-trkB.DN-mCherry, full name AAV-EF1a-DIO-trkB.DNTM570-mCherry (Addgene, \#121502), was constructed by PCR amplification of rat truncated trkB.T1 with the sequence downstream of the transmembrane domain replaced by the sequence of three alanine residues followed by a stop codon (from the plasmid pLTM570, gift from Lino Tessarollo, NIH). For visualization of neurons expressing trkB.DN, the stop codon was removed, and mCherry (from the plasmid pAAV-EF1a-double floxed-hChR2(H134R)-mCherry-WPRE-HGHpA, Addgene, \#20297) was fused to the $\mathrm{C}$ terminal of trkB.DN, using the same open reading frame. trkB.DN-mCherry was inserted between the two double floxed sites in the pAAV-EF1a-DIO plasmid backbone (Addgene plasmid \#20949), using NheI and AscI as restriction enzyme cloning sites. AAV5 particles $(8 \times 10 \mathrm{e} 12$ viral particles/ml $)$ were custom prepared by the Virus Vector Core Facility at the University of North Carolina at Chapel Hill. The AAV5-EF1a-DIO-eYFP (Addgene, \#27056; $3.3 \times 10 \mathrm{e} 12$ particles $/ \mathrm{ml}$ ), was produced by the Virus Vector Core Facility at the University of North Carolina at Chapel Hill.

\section{Viral injections}

A biosafety cabinet class 2 with sterile surgical environment was used for viral injections. Fixed to a Quintessential Stereotaxic Injector (Stoelting), the micropipette (graduated borosilicate glass capillary; Wiretrol I, Drummond) was filled with mineral oil, and $1 \mu \mathrm{l}$ of virus mixed with Fast Green FCF Solution 2.5\% (Electron Microscopy Sciences). The mouse was placed on a heating blanket $\left(37^{\circ} \mathrm{C}\right)$ in a stereotaxic apparatus (Harvard Apparatus) and anesthetized (2\% isoflurane). The mouse's reflexes were tested, and the isoflurane level was reduced to $1 \%$ or $1.5 \%$ over the course of the surgery. The scalp was shaved, and lidocaine $1 \%$ was injected subcutaneously at the site of surgery. The mouse was treated with analgesic buprenorphine $(0.2 \mathrm{mg} / \mathrm{kg})$ before skin incision, and the eyes were covered with eye lubricant (Viscotears, Novartis). Using a fresh scalpel blade, a single incision through the skin was made over the injection location. The connective tissue around the skull was gently removed for clear viewing of the bregma. Bilateral craniotomies were performed over the mPFC [anteroposterior (AP): $1.8 \mathrm{~mm}$, mediolateral (ML): $\pm 0.3 \mathrm{~mm}$, relative to bregma]. Sterile saline $0.9 \%$ was regularly 
applied over the skull while drilling to remove bone dust and control heat generation. The total diameter of the burr hole was not larger than the diameter of the drill bit $(\sim 100 \mu \mathrm{m})$. The remaining skull was removed using a syringe needle, exposing the dura that was thereafter carefully opened. The tip of the micropipette was lowered through the dura to reach the target location [prelimbic region; dorsoventral (DV): $-2 \mathrm{~mm}$ relative to bregma]; $0.5 \mu \mathrm{l}$ virus/hemisphere was injected at a rate of $0.1 \mu \mathrm{l} / \mathrm{min}$. The micropipette was kept in place for $10 \mathrm{~min}$ after each finished injection and thereafter slowly retracted. The skin was closed with small amounts of cyanoacrylate glue (Vetbond Tissue Adhesive, Henry Schein Medical) and carprofen $(5 \mathrm{mg} / \mathrm{kg}$, i.p.; Norocarp, Norbrook Laboratories) was given for analgesics before termination of anesthesia. The mouse was monitored until completely recovered and thereafter placed in the homecage. An additional dose of carprofen was administered $24 \mathrm{~h}$ after surgery.

\section{Tissue processing}

The mice were deeply anesthetized with pentobarbital and transcardially perfused with $1 \times \mathrm{PBS}$ ( $\mathrm{pH}$ 7.4) followed by $4 \%$ paraformaldehyde (PFA) in PBS (0.1 $\mathrm{M}$; $\mathrm{pH} 7.4$ ). The perfused brain was removed from the skull and postfixed with $4 \% \mathrm{PFA}$ in $0.1 \mathrm{M} \mathrm{PBS}$ at $4^{\circ} \mathrm{C}$ for $16 \mathrm{~h}$. For vibratome cutting, the brain was thoroughly washed in $0.1 \mathrm{M}$ PBS and thereafter coronally sectioned (40- $\mu \mathrm{m}$ thickness) using a vibratome (Leica VT1200S, Leica Microsystems, Nussloch $\mathrm{GmbH}$ ). The sections were stored in $1 \times$ PBS at $4^{\circ} \mathrm{C}$. For Cryosectioning, brains were after post-fixing cryoprotected in $10 \%$ sucrose in $1 \times$ PBS overnight and thereafter frozen using liquid carbon dioxide. The brains were sectioned (14- $\mu \mathrm{m}$ thickness) using a CryoStarTM NX70 Cryostat (ThermoFisher Scientific) and mounted on Superfrost Plus glass slides (ThermoFisher Scientific).

\section{Immunohistochemistry General protocol \\ Both vibratome cut (free-floating) and cryosectioned tissue were perme- abilized for $1 \mathrm{~h}$ with $0.3 \%$ Triton X-100 in $1 \times$ Tris-buffered saline (TBS; $38 \mathrm{~mm}$ Tris- $\mathrm{HCl}, 8 \mathrm{~mm}$ Trizma base, $120 \mathrm{~mm} \mathrm{NaCl}$ in extra pure water), blocked with $10 \%$ normal donkey serum in $1 \times$ TBST for $1 \mathrm{~h}$ at room temperature (RT), and thereafter incubated with primary antibodies (see antibody list) in $1 \times$ TBST at RT for $12-24 \mathrm{~h}$. The sections were then washed three times in $1 \times$ TBST, and incubated with a species-specific fluorophore-conjugated secondary antibody in $1 \times$ TBST for $3-5 \mathrm{~h}$ (see antibody list for details). The sections were next consecutively washed with $1 \times$ TBST, $1 \times$ TBS, and $1 \times$ PBS (10 min each). Vibratome cut sections were mounted on glass slides (Superfrost Plus, ThermoFisher Scientific). All sections were cover-slipped (ThermoFisher Scientific) using 50:50 glycerol:1× PBS. Images were acquired at 10 or $20 \times$ magnification with a fluorescent microscope (Leica DM6000B) connected to a Hamamatsu Orca- FLASH 4.0 C11440 digital camera (Hamamatsu, Hamamatsu City). The primary and secondary antibodies used are shown in Table 1 and Table 2, respectively.}

\section{Evaluation of cell-type-specific expression of AAV-DIO-trkB.DN-} $m$ Cherry

$40 \mu \mathrm{m}$ free-floating mPFC sections were stained with antibodies against PV and mCherry and mounted. Confocal images covering all six cortical layers were captured (LSM-800 Zeiss) using a $20 \times$ or $60 \times$ objective and identical pinhole, gain, and laser setting. The co-expression of PV and mCherry was quantified using the Cell Counter plugin of the software FIJI (Schindelin et al., 2012).

\section{GABA and $P V$ levels and trkB EC/IC ratio}

An antigen retrieval step was introduced before blocking during immunohistochemistry. For this, the free-floating sections were incubated for $5 \mathrm{~min}$ in $70^{\circ} \mathrm{C}$ warm sodium citrate buffer $(10 \mathrm{~mm}$ tri-sodium citrate in distilled water, $0.05 \%$ Tween $20, \mathrm{pH}$ 6), and thereafter washed in TBST (RT, $10 \mathrm{~min}$ ). Immunostaining was thereafter performed as described above. The sections were mounted on glass slides (Superfrost Plus, ThermoFisher Scientific) using SlowFadeGold antifade reagent (ThermoFisher Scientific). Confocal z-stack images (six images per stack) were captured (LSM-800
Zeiss) with a $60 \times$ objective and consistent settings. The corrected total cell fluorescence (CTCF) of GABA, PV, EC, and IC was measured using the software FIJI. For antibodies, see Table 1 and Table 2.

\section{Ex vivo electrophysiology}

The animal was deeply anesthetized and transcardially perfused with ice-cold cutting solution, containing the following: $40 \mathrm{~mm} \mathrm{NaCl}, 2.5 \mathrm{~mm}$ $\mathrm{KCl}, 1.25 \mathrm{~mm} \mathrm{NaH}_{2} \mathrm{PO}_{4}, 26 \mathrm{~mm}$ NaHCO, $20 \mathrm{~mm}$ glucose, $37.5 \mathrm{~mm}$ sucrose, 20 mM HEPES, $46.5 \mathrm{~mm}$ NMDG, $46.5 \mathrm{~mm} \mathrm{HCl}, 1 \mathrm{~mm}$ L-ascorbic acid, $0.5 \mathrm{~mm} \mathrm{CaCl}_{2}$, and $5 \mathrm{~mm} \mathrm{MgCl}_{2}$. The animal was then decapitated and the brain dissected out; $250-\mu \mathrm{m}$ thick coronal slices were vibratome cut (VT1200S, Leica) in ice-cold cutting solution, the slices were incubated in cutting solution at $34^{\circ} \mathrm{C}$ for $13 \mathrm{~min}$, and then maintained at RT in recording solution until recording, containing the following: $124 \mathrm{mM}$ $\mathrm{NaCl}, 2.5 \mathrm{~mm} \mathrm{KCl}, 1.25 \mathrm{~mm} \mathrm{NaH}_{2} \mathrm{PO}_{4}, 26 \mathrm{~mm} \mathrm{NaHCO}_{3}, 20 \mathrm{~mm}$ glucose, $2 \mathrm{mM} \mathrm{CaCl}_{2}$, and $1 \mathrm{~mm} \mathrm{MgCl}_{2}$.

For recording, the slices were superfused with extracellular solution kept at $33-35^{\circ} \mathrm{C}$. Neurons were visualized using a $60 \times$ objective (Olympus) and a DIC microscope (Scientifica), and fluorescent neurons identified using a pE-4000 LED light source (CoolLED). Patch pipettes (resistance 5-10 M $\Omega$, pulled using a P-87 Flaming/Brown micropipette puller, Sutter Instruments) were filled with low chloride internal solution containing the following: $130 \mathrm{~mm} \mathrm{~K}$-gluconate, $5 \mathrm{~mm} \mathrm{Na}$-phosphocreatine, $1.5 \mathrm{~mm} \mathrm{MgCl}_{2}, 10 \mathrm{~mm}$ HEPES, $5 \mathrm{~mm} \mathrm{Mg-ATP,} 0.35 \mathrm{~mm}$ Na-GTP, $1 \mathrm{~mm}$ EGTA, and $8 \mathrm{~mm}$ biocytin. For part of the paired recordings, a high-chloride internal solution was used, containing the following: 120 mм KCl, 5 mм Na2-phosphocreatine, $1.5 \mathrm{~mm} \mathrm{MgCl}_{2}, 10$ mм HEPES, 5 mM Mg-ATP, $0.35 \mathrm{~mm} \mathrm{Na-GTP,} 1 \mathrm{~mm}$ EGTA, and $8 \mathrm{~mm}$ biocytin. For voltage-clamp recordings in PNs, cesium-based intracellular was used, containing the following: $125 \mathrm{~mm} \mathrm{CsMeSO}, 4.1 \mathrm{~mm}$ QX-314, $4 \mathrm{~mm}$ Na2-phosphocreatine, $10 \mathrm{~mm}$ HEPES, $5 \mathrm{~mm}$ Mg-ATP, $0.35 \mathrm{~mm} \mathrm{Na}$ GTP, 5 mm EGTA, and $0.75 \mathrm{~mm} \mathrm{CsOH}$.

Signals were recorded with an Axon MultiClamp 700B amplifier and digitized at $20 \mathrm{kHz}$ with an Axon Digidata 1550B digitizer (Molecular Devices). Access resistance and pipette capacitance were compensated. Excitatory spontaneous currents were recorded in voltage-clamp mode from PNs (identified by soma shape and lack of fluorescence) at a holding potential of $-70 \mathrm{mV}$, and subsequently, inhibitory spontaneous currents were recorded at $0 \mathrm{mV}$. To assess passive and active membrane properties, neurons recorded in current-clamp mode were held at a membrane potential of $-70 \mathrm{mV}$. The rheobase current was determined by applying near-threshold current steps, followed by steps proportional to the rheobase current with a duration of $1 \mathrm{~s}$. An additional small hyperpolarizing step $(30 \mathrm{pA})$ with a duration of $300 \mathrm{~ms}$ was used to determine the time constant and to monitor the access resistance throughout the recording. During paired recordings, a PV neuron (identified by red or green fluorescence and AP pattern) and a PN (identified by soma shape and AP pattern) were recorded in current-clamp. For PPR analysis, a pair of APs were evoked in the PV neuron, with a 50-ms interval between the two APs and a $10 \mathrm{~s}$ interval between sweeps.

Frequency of spontaneous synaptic events, intrinsic electrical properties, paired-pulse ratio, IPSP amplitude, and IPSP slope were extracted using custom-written MATLAB (The MathWorks) scripts (written by Janos Fuzik and Josina A van Lunteren).

The EPSC and IPSC frequency in PNs was determined by counting the number of synaptic events that occurred during a 3-min recording. EPSP frequency in PV interneurons was determined by counting the number of EPSPs that occurred during 20-60 s, while the PV interneuron was at $-70 \mathrm{mV}$. The AP characteristics were determined from the first spike that occurred at rheobase current stimulation. Firing rate and adaptation were determined from a current injection of approximately twice the rheobase current. MATLAB script (van Lunteren and Fuzik, 2020), as well as boundedline (Copyright 2010, Kelly Kearney), plotSpread (Copyright 2017, Jonas), and normality test (Öner and Kocakoç, 2017) were used to determine maxima adaptation.. In short, the time points of maximal and minimal immediate firing rate were found, and the average firing rate was determined over a time period of $110 \mathrm{~ms}$ around the maximum and minimum; the ratio between the 
Table 1. Primary antibodies

\begin{tabular}{|c|c|c|c|c|c|}
\hline Analysis & Target & Host & Dilution & Company & Catalog \# \\
\hline \multirow[t]{2}{*}{ Virus specificity and efficiency } & PV & Guinea pig & 1:1000 & Synaptic Systems & 195004 \\
\hline & mCherry & Chicken & $1: 500$ & Novus Biologicalalals & NBP2-25158 \\
\hline \multirow[t]{4}{*}{ Sholl analysis and axonal bouton counting } & PV & Guinea pig & 1:1000 & Synaptic Systems & 195004 \\
\hline & mCherry & Chicken & $1: 1000$ & Novus Biologicalalals & NBP2-25158 \\
\hline & Gephyrin & Mouse & 1:500 & Synaptic Systems & 47021 \\
\hline & eGFP & Chicken & $1: 1000$ & Abcam & ab13970 \\
\hline \multirow{3}{*}{ GABA levels } & Gephyrin & Mouse & $1: 500$ & Synaptic Systems & 47021 \\
\hline & mCherry & chicken & $1: 500$ & Novus Biologicalalals & NBP2-25158 \\
\hline & eGFP & Chicken & $1: 1000$ & Abcam & ab13970 \\
\hline \multirow[t]{3}{*}{ trkB.IC/EC ratio } & PV & Guinea pig & $1: 1000$ & Synaptic Systems & 195004 \\
\hline & trkB-extracellular (amino acids 32-429) & Rat & $1: 300$ & Antibodies-online & ABIN1983534 \\
\hline & trkB-intracellular (amino acids 671-720) & Rabbit & $1: 300$ & Antibodies-online & ABIN1532221 \\
\hline
\end{tabular}

Table 2. Secondary antibodies

\begin{tabular}{|c|c|c|c|c|c|c|}
\hline \multirow[b]{2}{*}{ Analysis } & \multicolumn{6}{|c|}{ Secondary antibody } \\
\hline & Target & Fluorophore & Host & Dilution & Company & Catalog \# \\
\hline \multirow[t]{3}{*}{ Virus specificity and efficiency } & Guinea pig & Alexa Fluor 488 & Donkey & $1: 500$ & Jackson ImmunoResearch & $706-545-148$ \\
\hline & Rabbit & $\mathrm{Cy3}$ & Donkey & $1: 500$ & Jackson ImmunoResearch & $711-175-152$ \\
\hline & Guinea pig & Суз & Donkey & $1: 1000$ & Jackson ImmunoResearch & $706-175-148$ \\
\hline \multirow[t]{9}{*}{ Sholl analysis and axonal bouton counting } & Rabbit & DyLight 405 & Donkey & $1: 500$ & Jackson ImmunoResearch & $711-475-152$ \\
\hline & Guinea pig & Alexa Fluor 488 & Donkey & $1: 1000$ & Jackson ImmunoResearch & $706-545-148$ \\
\hline & Chicken & Alexa Fluor 488 & Donkey & $1: 1000$ & Jackson ImmunoResearch & $703-545-155$ \\
\hline & Rabbit & $\mathrm{Cy3}$ & Donkey & $1: 1000$ & Jackson ImmunoResearch & $711-175-152$ \\
\hline & Guinea pig & Cу3 & Donkey & $1: 1000$ & Jackson ImmunoResearch & $706-175-148$ \\
\hline & Mouse & Cy5 & Donkey & $1: 500$ & Jackson ImmunoResearch & $715-175-151$ \\
\hline & Streptavidin & Alexa Fluor 633 & & $1: 50000$ & ThermoFisher Scientific & S21375 \\
\hline & Streptavidin & Alexa Fluor 488 & & 1:1000 & Jackson ImmunoResearch & 016-540-084 \\
\hline & Streptavidin & $\mathrm{Cy3}$ & & $1: 1000$ & Jackson ImmunoResearch & $016-160-084$ \\
\hline \multirow[t]{6}{*}{ GABA levels } & Guinea pig & DyLight 405 & Donkey & $1: 500$ & Jackson ImmunoResearch & $706-475-148$ \\
\hline & Rabbit & Cy 5 & Donkey & $1: 500$ & Jackson ImmunoResearch & $711-175-152$ \\
\hline & Mouse & Alexa Fluor 488 & Donkey & $1: 500$ & Jackson ImmunoResearch & $715-545-151$ \\
\hline & Mouse & $\mathrm{Cy3}$ & Donkey & $1: 500$ & Jackson ImmunoResearch & $715-165-151$ \\
\hline & Chicken & Cy3 & Donkey & $1: 500$ & Jackson ImmunoResearch & $703-165-155$ \\
\hline & Chicken & DyLight 405 & Donkey & $1: 500$ & Jackson ImmunoResearch & $703-475-155$ \\
\hline \multirow[t]{6}{*}{ trkB.IC/EC ratio } & Guinea pig & DyLight 405 & Donkey & $1: 500$ & Jackson ImmunoResearch & $706-475-148$ \\
\hline & Rat & Alexa Fluor 488 & Donkey & $1: 500$ & Jackson ImmunoResearch & $712-545-153$ \\
\hline & Rabbit & Cy5 & Donkey & $1: 500$ & Jackson ImmunoResearch & $711-175-152$ \\
\hline & Rabbit & $\mathrm{Cy3}$ & Donkey & $1: 500$ & Jackson ImmunoResearch & $711-175-152$ \\
\hline & Rat & $\mathrm{Cy3}$ & Donkey & $1: 500$ & Jackson ImmunoResearch & $712-165-153$ \\
\hline & Rabbit & Alexa Fluor 488 & Donkey & $1: 500$ & Jackson ImmunoResearch & $711-545-152$ \\
\hline
\end{tabular}

average maximal firing rate and the average minimal firing rate is the maximal adaptation. The amplitude of the first IPSP was defined as the difference between the baseline, measured over $15 \mathrm{~ms}$ ( $5 \mathrm{~ms}$ before the evoked spike), and the peak of the first IPSP. The slope of the first IPSP was fitted with an exponential function using physfit (Copyright 2006, Daniel Wagenaar). To determine the PPR, the amplitude of the second IPSP was determined by subtracting the fitted tail of the first IPSP from the peak of the second IPSP.

Tissue clearing for Sholl analysis and counting of axonal boutons Slices containing biocytin filled PV interneurons were fixed in 4\% PFA (in $0.1 \mathrm{M} \mathrm{PB}, \mathrm{pH} 7.8$ ) overnight at $4^{\circ} \mathrm{C}$. The following day, the slices were repeatedly washed in $\mathrm{PB}$ and thereafter incubated for $24 \mathrm{~h}$ in CUBIC reagent 1 (25 wt $\%$ urea, $25 \mathrm{wt} \%$ N,N,N',N'-tetrakis(2-hydroxypropyl) ethylenediamine, $15 \mathrm{wt} \%$ polyethylene glycol mono-p-isooctylphenyl ether/Triton X-100). CUBIC is used to clear tissues by promoting the release of heme while maintaining a moderately alkaline environment (Susaki et al., 2014). The slices were thereafter washed three times in PBT (0.1 M PB containing 0.3\% Triton X-100) and blocked for $3 \mathrm{~h}$ in $5 \%$ donkey serum in PBT. The slices were incubated with primary antibodies in PBT (see antibody list) for $48 \mathrm{~h}$ at $4^{\circ} \mathrm{C}$. After washing with PBT the slices were incubated for $24 \mathrm{~h}$ at $4^{\circ} \mathrm{C}$ in PBT containing $0.001 \%$ secondary antibodies (see antibody list) and $0.002 \%$ streptavidin conjugated to Cy5 fluorophore (ThermoFisher Scientific). The slices were washed in $\mathrm{PB}$ for $3 \mathrm{~h}$ and thereafter incubated in CUBIC reagent 2 [50 wt\% sucrose, $25 \mathrm{wt} \%$ urea, $10 \mathrm{wt} \% 2,2^{\prime}, 2^{\prime \prime}$-nitrilotriethanol, and $0.1 \%(\mathrm{v} / \mathrm{v})$ Triton $\mathrm{X}$ 100 ] overnight and thereafter mounted on glass slides (Superfrost Plus, ThermoFisher Scientific). 


\section{Sholl analysis and counting of axonal boutons}

Tiled confocal z-stack images (ca 60 images/stack; LSM-800, Zeiss) were acquired of immunohistochemically stained biocytin-filled PV interneurons at $20 \times$. The images were processed with Fiji software, and the neurons reconstructed using the plugin Simple Neurite tracer, a semiautomated framework for tracing of neurons (Longair et al., 2011). The neurites were marked either as axons or dendrites, and their length was measured. Sholl analysis was used to characterize the neuronal arbors, in which a series of concentric (sampling) spheres (10- $\mu \mathrm{m}$ interval between the radii) were created around the middle point (the soma of the traced neuron; Ferreira et al., 2014). The algorithm counts how many times neurites intersect the sampling spheres. In order to perform Sholl analysis on specific regions of the neurite arbor, we used the conventional inside-out Sholl analysis where neurites that extend from the cell body are defined as primary neurites, neurites emanating from primary neurites defined as secondary neurites, and so on. Neurites classified as tertiary or higher were grouped together (O'Neill et al., 2015). Quantification of axonal boutons (putative synaptic terminals) was done in the same images. For each neuron, three secondary axons with a length of 100$300 \mu \mathrm{m}$ were randomly picked and all boutons were counted. The average number of boutons $/ 100 \mu \mathrm{m}$ was calculated for each axonal path. For calculation of the boutons' size, the boutons were processed using the Pixel Classification workflow from the software ilastik (version 1.3.3; Berg et al., 2019), and the results exported into the FIJI software where the average sizes of labeled pixels were calculated using the Analyze Particles plugin.

For immunohistochemical detection and quantification of axonal boutons of biocytin-filled mPFC PV basket neurons expressing trkB.DN ( $n=12$ axonal paths, in 3 trkB.DN mice) or eYFP ( $n=12$ axonal paths, in 2 eYFP mice) and gephyrin (scaffold protein for GABAergic synapses), confocal z-stack images ( $\sim 12$ images/stack; $z$-step of $\sim 0.4 \mu \mathrm{m})$ of biocytin-filled axons were acquired with a $60 \times$ oil-immersed objective and a numerical zoom of $2.5 \times$. The images were then processed with Imaris 7.4 software (Bitplane). We performed a spot detection of the gephyrin labeling, while for axonal boutons reconstruction, biocytinpositive boutons were reconstructed as $3 \mathrm{D}$ structures using "surface objects." To analyze the proximity between the axonal boutons and the closest gephyrin spot, we used the Distance Transformation plugin and obtained the distances from gephyrin spot centroid to presynaptic bouton center. We calculated the average distance per axonal path, as well as the percentage of gephyrin/biocytin boutons, using a distance threshold of $1 \mu \mathrm{m}$ from spot centroid to presynaptic bouton center.

\section{snRNA-seq}

Vgat-Cre mice (Jax stock \#028862) were crossed to mice with Credependent expression of histone-B associated eGFP (H2B-eGFP; RIKEN LARGE, CDB0203K) to generate mice with nuclear eGFP expression in Vgat-expressing neurons (Vgat-Cre:H2B-eGFP mice). The animals ( $n=2$ male, nine weeks old) were killed with an overdose of isoflurane, and the brains were rapidly extracted from the skull and immersed in an ice-cold aCSF solution. The brains were immediately sectioned $(300 \mu \mathrm{m})$ in ice-cold aCSF using a vibratome (VT1200S, Leica), and the sections placed in a Petri dish with ice-cold Leibovitz's L-15 medium (Invitrogen, ThermoFisher Scientific). The PFC was dissected out from the sections and the tissue stored in $1 \mathrm{ml}$ ice-cold Leibovitz's L-15 medium containing 1- $\mu$ l SUPERase RNase inhibitor (20 U/ $\mu$ l, ThermoFisher Scientific). Specifically, the neuron nuclei were isolated using a nuclear isolation protocol (Yeung et al., 2014). The tissue was homogenized in a 2-ml lysis buffer using a Dounce tissue grinder ( $7 \mathrm{ml}$, VWR). After addition of a $1.8 \mathrm{~m}$ sucrose solution $(4 \mathrm{ml})$, the homogenized solution was added onto a sucrose cushion $(2 \mathrm{ml})$ in a $10 \mathrm{ml}$ Ultra-Clear centrifuge tube (Beckman Coulter). Separation of nuclei from the tissue was done by centrifugation $\left(26,500 \times g\right.$ at $4^{\circ} \mathrm{C}$ for $\left.1.5 \mathrm{~h}\right)$ and after discarding of the supernatant, the nuclei pellet was resuspended in a $500-\mu$ l nuclear resuspension buffer. Single nuclei were isolated using fluorescence-activated cell sorting (FACS) by their GFP emission profile, and sorted into 384 well-plates containing 2.3- $\mu$ l ice-cold lysis buffer. Plates containing nuclei were straightaway frozen on dry ice and stored on $-80^{\circ} \mathrm{C}$ until additional processing. cDNA libraries were constructed and sequenced using a Smart-seq2 protocol (Picelli et al., 2013). Concisely, after lysis of the nuclei, the poly A RNA reverses were transcribed, followed by PCR preamplification and purification. Tagmentation of the cDNA was followed by PCR amplification of the fragments and purification. The final cDNA libraries were sequenced using Illumina HiSeq 2000 (Illumina). The reads were mapped and aligned to the mouse genome $(\mathrm{mm} 10)$ and gene expression values were calculated as count values for each transcript. Analysis was performed on count values of the exome per nucleus. The sequencing data counts (from $n=648$ individual nuclei) were analyzed using the Seurat package in $\mathrm{R}$ (versions 1.4 .0 and 2.3.4; Butler et al., 2018), as published before (Märtin et al., 2019). The count data were normalized $(\log 2)$ and $z$ score of $\log ($ variance/mean) was used to identify variance genes. Cut off for high-low gene level was established at $>1 z$ score. After performing a principal component analysis (PCA) to obtain the genes that are differentially expressed throughout the population, the significant Principal Components in the dataset were determined after random sampling with 1000 replicates and a projected PCA was used to expand the gene list and to avert losing possible marker genes. The resultant list of genes (3000 number of unique genes per nucleus from 648 Vgat-Cre:H2B-GFP nuclei) was once more analyzed for PCs and randomly sampled (1000 replicates). The resulting significant PCs were implemented into a nonlinear dimensionality reduction ( $t-S N E$ ) analysis. A subsequent density-based clustering was implemented and markers per cluster were identified based on their distinctive expression.

\section{Behavioral testing}

All behavioral tests, and any included electrophysiology, and their analyses were performed by an experimented blind to the animal ID (trkB. $\mathrm{DN}$ or eYFP mice). All mice were habituated to the experimenter for 1$2 \mathrm{~d}$ before the first test day and transported to the testing room at least 1 $\mathrm{h}$ before any procedures to facilitate adaptation to the surroundings. All tests were conducted during the light phase.

\section{Resident-intruder procedure}

The home cage $(36 \times 18 \times 12 \mathrm{~cm})$ of the resident mouse was used for the resident-intruder procedure. The cage top was replaced by a transparent acrylic piece to allow video recording with a CCD camera. At the start of the session, the food and enrichment were removed (original bedding remained), and the resident was free to explore the cage for $1 \mathrm{~min}$. A juvenile (three- to four-week-old, group-housed) male mouse was introduced in a corner opposite to the current resident's location, kept in the cage for $4 \mathrm{~min}$, and thereafter removed. Social and non-social behaviors (Winslow, 2003) were scored post hoc from the videos using a custom-made MATLAB (The MathWorks): exploration (searching environment or rearing along the cage border), digging (fast alternating movements of the forepaws scraping back material), grooming (mice in sitting position with licking of the fur, grooming with the forepaws, or scratching with any limb), tail rattling (fast waving movements of the mouse tail), sniffing (body trunk, anogenital and nosing exploration of the intruder), and aggressive (attack, fighting as kicking, biting, and wrestling) behaviors.

\section{Elevated plus-maze test}

Anxiety was probed in an elevated $(73.5 \mathrm{~cm}$ above the floor) plus-maze with four arms (each arm: length; $40 \mathrm{~cm}$, width; $7 \mathrm{~cm}$ ), with two of the arms holding $15-\mathrm{cm}$-high opaque walls (closed arms). At the start of the test, the mouse was placed in the center $(5 \times 5 \mathrm{~cm})$ of the maze, facing one of the closed arms. The mouse was allowed to freely explore the maze for $5 \mathrm{~min}$, and the behavior was recorded with a CCD camera. The behavior was analyzed with Biobserve Viewer Software (Biobserve $\mathrm{GmbH}$ ) to score the number of entries and the time spent in the center, the closed arms, and the open arms, respectively. Head dips (downward movement of the head toward the floor in the room performed while in the open arms, a sign of reduced levels of anxiety) and stretches attended postures (stretching toward the open area from inside the closed area). The maze was cleaned with $70 \%$ ethanol and wiped dry with paper towels between each mouse. 


\section{Open-field test}

Locomotion and anxiety were scored in open field boxes $(46 \times 46 \times$ $30 \mathrm{~cm}$ with 16 light beam arrays, ActiMot2, TSE Systems). The breaking of light beams was used to score the location and activity of the animal. In each session, a single mouse was placed in the box (near a wall) and allowed to explore the box for $60 \mathrm{~min}$ freely. The total distance traveled was scored, as were the time spent, number of visits, and the velocity $(\mathrm{cm} / \mathrm{s})$ during visits, in the center of the box (imaginary square with $25 \%$ of the open-field area). The box was cleaned with $70 \%$ ethanol and wiped dry with paper towels between each mouse.

\section{Acute in vivo electrophysiology}

The mouse was anesthetized with urethane $(1.1 \mathrm{~g} / \mathrm{kg}$ in sterile saline $0.9 \%$, i.p.) $1 \mathrm{~h}$ before start of the recordings, fixed in the stereotaxic apparatus on a heating blanket with feedback loop able to maintain the body temperature at $37 \pm 0.5^{\circ} \mathrm{C}$ (Harvard Apparatus) and kept under isoflurane anesthesia $(0.25 \%)$ during surgery. A craniotomy was made at the site of the drill hole made for viral injection, large enough to allow positioning of a four-shank silicon probe (A4x2-tet-5 mm150-200-312-A32, Neuronexus), which was coupled to an adapter for connecting a 32 channel headstage preamplifier (Neuralynx), linked to a Digital Lynx 4SX system (Neuralynx). The dura was carefully removed. Small amounts of saline $(0.9 \%)$ were continuously dropped on the craniotomy to prevent brain surface dryness. An additional craniotomy was made over the cerebellum for fixing of the ground-screw used as reference. A fluorescent lipophilic dye (DiI, Vybrant DiI Cell-Labeling Solutions, ThermoFisher Scientific) was painted on the electrode shank for post hoc detection of the probe tracts. We let the electrode to air-dry for at least $5 \mathrm{~min}$. The silicon probe was carefully positioned in the mPFC (AP: $1.75 \mathrm{~mm}$, ML: $\pm 0.3 \mathrm{~mm}, \mathrm{DV}:-2 \mathrm{~mm}$ ) and using the Cheetah 5.0 software (Neuralynx), we monitored in real-time the electrophysiological signal for $30 \mathrm{~min}$ to allow the brain tissue to recover and optimize the recording condition. Two different recordings were performed. First, $1 \mathrm{~h}$ of recording was performed to monitor different oscillatory states induced by urethane (data not shown). Second, a 6-min recording was performed to assess $\mathrm{mPFC}$ modulation induced by a tail pinch. After a 2-min baseline recording, a tail pinch (7 s) was manually applied using a plastic-coated surgical plier, and electrophysiological activity was recorded for the following $4 \mathrm{~min}$. The recorded electrical signals were divided, preamplified $(1000 \times)$ and filtered for LFP and single-unit activity. The biological signal was sampled at $32 \mathrm{kHz}$ and bandpass filtered between $600-6000 \mathrm{~Hz}$ to record spikes, and between $0.5-500 \mathrm{~Hz}$ to record LFPs. After the recordings, the animal was deeply anesthetized with pentobarbital, transcardially perfused, and the brain was recovered for post hoc detection of the probe location using SHARP-Track (Shamash et al., 2018) and SBA Composer (Bakker et al., 2015).

\section{Chronic in vivo electrophysiology}

FlexDrive construction and implantation

For chronic in vivo electrophysiology, flexDrive implants were constructed with seven tetrodes (Voigts et al., 2013). Custom-made tetrodes consisted of four twisted fine wires (polyimide insulated ni-chrome wire, $12 \mu \mathrm{m}$, Sandvik-Kanthal) that were gold-plated to reduce the impedance to $0.2-0.4 \mathrm{M} \Omega$ at $1 \mathrm{kHz}$. Seven movable tetrodes were loaded into medical-grade polyimide carrier tubes (0.005-inch OD, Phelps Dodge) in the flexDrive. An extra wire was installed for electromyography (EMG) recording.

For implantation of the flexDrive, the mice were anesthetized with isoflurane (2\%) and the body temperature maintained at $37^{\circ}$ with a temperature controller system. The animals were fixed in a stereotaxic frame and a hole was drilled through the skull $(1.75 \mathrm{~mm}$ anterior to bregma and $0.30 \mathrm{~mm}$ lateral to midline). The flexDrive was positioned above the craniotomy and the tetrodes gradually lowered to the prelimbic area (1.25 mm ventral to the brain surface). Two miniature anchoring screws (Fine Science Tools) were used to attach the flexDrive to the skull (one on the anterior and one on the posterior part of the skull). Two Teflon coated stainless steel wires (0.005-inch bare, AM Systems) from the electrode interface board of the flexDrive were connected to the screws for grounding. The flexDrive was secured onto the skull using light curing dental adhesive (OptiBond FL, Kerr) and cement (Tetric EvoFlow, Ivoclar Vivadent). The animals were injected with analgesic $(0.1 \mathrm{mg} / \mathrm{kg}$ buprenorphine, s.c. $)$ at the beginning of surgery and carprofen $(5 \mathrm{mg} / \mathrm{kg}$; Norocarp, Norbrook Laboratories) was intraperitoneally injected at the end. The animal was monitored until completely recovered from the anesthesia and thereafter single-housed. An additional dose of carprofen was administered $24 \mathrm{~h}$ after surgery.

Electrophysiological recordings during the resident-intruder procedure The resident intruder procedure was performed as described above with the exception that the resident was connected to the Neuralynx Cheetah 64 system (Digital Lynx 4SX, Neuralynx) and allowed to explore the cage freely for $9 \mathrm{~min}$ before the intruder was introduced (baseline). The intruder was introduced [social interaction (SI)] and removed after $4 \mathrm{~min}$. The recordings continued for $10 \mathrm{~min}$ after the intruder had been removed (post-SI). For synchronization of recorded neural data, a custom-made Python 3.7 (Python Software Foundation) script was used to control the CCD camera connected to a USB-controlled Arduino microprocessor (Arduino Uno Rev3) that outputs a train of voltage pulses (TTL trigger directly to the neural acquisition system) during electrophysiology recording.

\section{Electrolytic lesions}

Electrolytic lesions were performed after the chronic recordings for mapping of the tetrode recording sites. The mouse was anesthetized with isoflurane (3\%), a crocodile clamp was fixed on one of the ears of the animal. A 30-mA current was applied between the crocodile clamp and the target electrode channel on the tetrodes for $30 \mathrm{~s}$ per channel to produce an electrolytic lesion. The mouse was thereafter perfused, and the brain recovered for post hoc detection of the location of the tetrodes using SHARP-Track (Shamash et al., 2018) and SBA Composer (Bakker et al., 2015).

\section{Data analysis}

Data analysis was conducted using custom software written in MATLAB (The MathWorks).

\section{Unit sorting and classification}

Single-units were manually sorted and identified by various spike waveform features (energy, peak, area, valley, peakValleyRatio, spike width, peakIndex, peak6to11, and principal components) using the offline spike-sorting toolbox MClust version 4.4 (A.D. Redish; available at http://redishlab.neuroscience.umn.edu/MClust/MClust.html). Only well-isolated units (Schmitzer-Torbert et al., 2005) with isolation distance $>15$, L-ratio $<0.2$, and the spikes $<0.01 \%$ at interspike interval (ISI) $<2 \mathrm{~ms}$ were included in the data analysis.

The unit classification was performed according to Ardid et al. (2015). First, we verified whether the peak-to-trough duration of all units followed a bimodal distribution through a Calibrated Hartigans' dip test analysis. Second, two Gaussian mixture models (GMMs) were fit to the peak-to-trough duration for objective classification in wide-spiking (WS) putative PNs or narrow-spiking (NS) putative interneurons, resulting in a WS-likelihood and an NS-likelihood for all units. For WS classification, only units with a WS-likelihood 10 times larger than the NSlikelihood were selected. In a similar manner, for NS classification only units with a NS-likelihood 10 times larger than the WS-likelihood were selected. Units that did not meet this criterion were not included in analysis (and categorized as unclassified). The classification was performed with an Open Source toolbox available at https://bitbucket.org/sardid/ waveformanalysis.

\section{Analysis of mPFC activities during SI}

Recordings of LFP and single-unit activity were done during three separated periods: after a baseline period of $9 \mathrm{~min}$, an intruder mouse was placed into the home cage and the SI lasted a total of $4 \mathrm{~min}$. After the 
removal of the intruder, the recording continued for an extra period of $10 \mathrm{~min}$.

For the analysis of the mPFC oscillations, we first calculated the LFP's root mean squared (RMS) envelope and established a manual threshold for movement detection to avoid movement-induced noises. Then, the LFP signal was segmented into $10 \mathrm{~s}$ epochs, and epochs presenting values above the manual threshold were removed. We next calculated the mean normalized LFP power for different frequencies $(\theta: 6-$ $10 \mathrm{~Hz}, \beta: 12-24 \mathrm{~Hz}$, and $\gamma$ range: $30-95 \mathrm{~Hz}$ ) using a bootstrap method. For each iteration, random epochs were included [epochs: $n=41$ for baseline, $n=21$ for SI, $n=51$ for post-SI per animal group (eYFP and trkB.DN mice)]. The same epoch could be randomly included in several iterations. After the bootstrap resampling, the normalized power was calculated. For this, the power spectral density (PSD) was estimated for the bootstrapped epochs using Welch's method (MATLAB algorithm), with $1 \mathrm{~s}$. Hamming window, with $50 \%$ overlap and a 5120 fast-Fourier transform (FFT) points. Normalized power was obtained by dividing the PSD estimation by the integrated power over all frequencies $(0-200 \mathrm{~Hz})$. The PSDs for bootstrapped epochs were then averaged for the eYFP and trkB.DN mice, respectively, and the confidence interval of the PSD distribution of each frequency band was calculated. The bootstrapped PSDs were compared between the two animal groups, and intragroup, and the confidence interval of group differences was calculated. PSDs differences with confidence intervals that contained the value "zero" were not considered significant.

For single-unit activity analysis, firing rate histograms were calculated for each individual WS neuron during SI, with a 4-s bin size and convoluted with a Gaussian Kernel of $12 \mathrm{~s}$. The firing rate was z-scored against the baseline period. Only WS neurons with a firing rate of at least 0.1 spike/s during baseline were selected to further analysis. The mean $\mathrm{z}$ scored firing rate was compared between groups, as well as the mean firing rate (in spike/s) and the coefficient of variation (CV; defined as the SD divided by the mean of ISI) of baseline activity.

Neurons were classified as negatively modulated, not modulated, and positively modulated by comparing all bins during the baseline with the first 2 min of SI with a Wilcoxon rank-sum test. Neurons that presented a different distribution during SI in comparison with the baseline period $(p<0.05)$ and a higher mean firing rate were considered positively modulated neurons, while neurons that presented a different distribution and a lower average firing rate were considered as negatively modulated. Neurons that did not present different distributions before and during SI were considered as not modulated. Proportion and mean zscored firing rate of negatively modulated, not modulated, and positively modulated were compared between groups.

Mice movements during SI were analyzed using Deeplab Cut (Mathis et al., 2018). Four points over the mouse head (including the flexDrive) were used for position tracking. Training was performed with 20 frames of each recording using a ResNet 152 pretrained network, performing 900,000 iterations. After tracking analysis, the average velocity (in pixel/s) during baseline, SI, and post-SI, was inferred from the average position between all markers.

\section{Analysis of mPFC activities during tail pinch}

The mean firing rate was calculated for each individual WS neuron at three different intervals: $-2 \mathrm{~min}$ to tail pinch (baseline; $-2-0 \mathrm{~min}$ ), from tail pinch to $2 \mathrm{~min}$ after start of tail pinch ( $0-2 \mathrm{~min})$, and from $2 \mathrm{~min}$ to $4 \mathrm{~min}$ after tail pinch (2-4 min). Spike trains were discretized in 1-s bins, convolved with a Gaussian Kernel of $4 \mathrm{~s}$. Firing rate was thereafter $\mathrm{z}$ scored in relation to the baseline. Only neurons with mean firing rate higher than 0.1 spike/s during baseline were included in further analysis. All further analysis was conducted as for MPFC activities during SI.

\section{Neural trajectory analysis}

For analysis of neuronal population trajectories, the first two principal components of the z-scored firing rates of WS neurons were calculated with a PCA for each temporal bin (SI: bins of $4 \mathrm{~s}$; tail pinch: bins of $1 \mathrm{~s}$; Hamm et al., 2017; Levy et al., 2019; Pessoa, 2019). Projecting the first and second principal components in a $2 \mathrm{D}$ space, we compared the variability of resulting clusters representing task intervals (SI: baseline, SI and post-SI; tail pinch: $-2-0,0-2$, and 2-4 min after tail pinch) in terms of Euclidean Distance. The Euclidean distance was calculated as the pairwise distance values of PC1 and PC2 for each cluster centroid. Using a cumulative distribution function, we compared the probability distribution of Euclidean Distance for each cluster between trkB.DN and eYFP mice with a Kolmogorov-Smirnov test for continuous distributions. To analyze the trajectories of specifically the negatively, positively and not modulated neurons for each task interval, a bootstrapped version of the trajectory analysis was performed with 10,000 iterations. At each iteration, a random sample of neurons (with reposition, i.e., the same neuron could be randomly included in several iterations) were selected, and the resulting first and second PCs of $\mathrm{z}$-scored firing rate were calculated. Different from the analysis performed with all WS neurons, here, the average Euclidean distance was calculated for each iteration, resulting in a distribution of average Euclidean distances for the negatively, positively, and not modulated WS neurons.

\section{Experimental design and statistical analysis}

Masking was used during AAV injections (independent experimenter aliquoted the viruses), data collection (in vivo electrophysiological recordings and behavior) and data analysis for behavior. For ex vivo electrophysiology and immunohistochemistry experiments, masking was not used during data collection, but used in data analysis. A D'Agostino and Pearson test or Kolmogorov-Smirnov was used to assess the homogeneity of variance. If the data fulfilled the criteria for normal distribution, we analyzed the data using parametric statistics. If the data did not pass the normality test, we used the Wilcoxon rank-sum test or the Wilcoxon matched-pairs signed-rank test. For cumulative probability distribution, the Kolmogorov-Smirnov test was used. Only values of $p<0.05$ were considered statistically significant. For bootstrapped analysis, only confidence intervals not containing zero were considered statistically significant. Statistical analyses were performed using GraphPad Prism version 8.00 (GraphPad Software) or MATLAB. More information on experimental design and statistical analysis for each specific figure is reported in Extended Data Fig. 1-1, including sample size, variable description, and statistics reports.

\section{Results}

\section{Targeting of trkB.DN to mPFC PV interneurons in adult mice}

We used snRNA-seq (Märtin et al., 2019) to investigate the expression of Ntrk2 (gene encoding for all trkB isoforms) and $B d n f$ in Pvalb-expressing interneurons in the adult mPFC. Vgatexpressing neurons in the $\mathrm{MPFC}$ of adult mice were isolated using FACS, and the molecular profile was analyzed ( $n=648$ nuclei; Fig. 1A; Materials and Methods). Clustering of gene expression data revealed 255 nuclei expressing Pvalb, with 253 nuclei co-expressing Ntrk2. Two clusters (14 and 15) expressed high levels of Pvalb (Fig. 1B), with 98.9\% (173/175) and 100\% $(12 / 12)$ of the nuclei, respectively, also expressing Ntrk2 (Fig. $1 C)$. Bdnf was expressed in $2.9 \%(5 / 175)$ and $0 \%(0 / 12)$ of the nuclei in the two clusters (Fig. 1D). The data confirms the expression of Ntrk2, and the absence of autocrine mechanisms of BDNF (Cellerino et al., 1996; Gorba and Wahle, 1999) in prefrontal PV interneurons. The data further supports the notion that PNs modulate GABAergic inhibitory activity of presynaptic interneurons through paracrine actions of BDNF (Jiao et al., 2011).

To allow for spatially restricted and cell-type-specific inhibition of BDNF/trkB signaling in the adult brain, we generated an AAV vector with Cre-dependent expression of trkB.DN (AAVDIO-trkB.DN-mCherry). Our trkB.DN construct holds intact extracellular and transmembrane domains, but the intracellular domain has been replaced by the monomeric red fluorescent protein mCherry (Fig. 1E; Materials and Methods). Thus, trkB. 
A
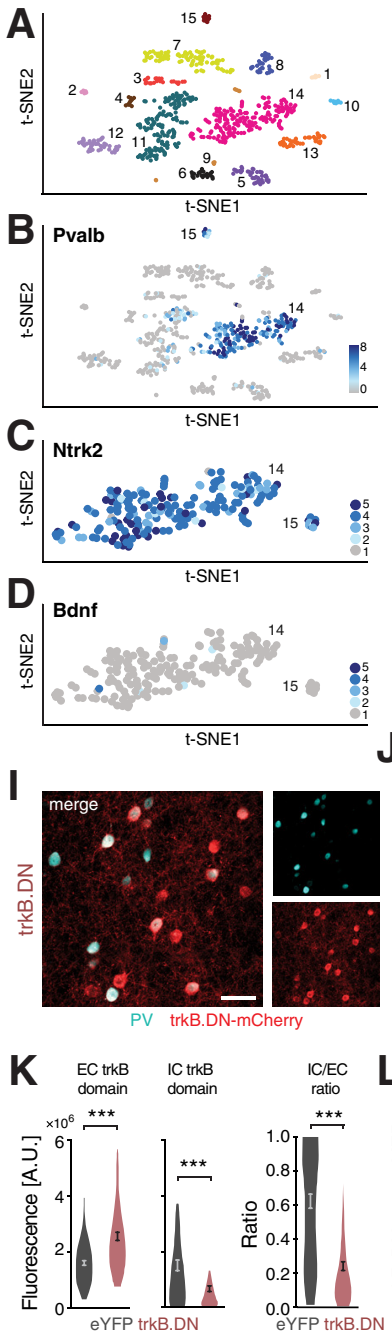

E

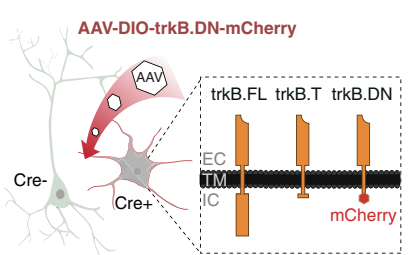

$\mathbf{F}$

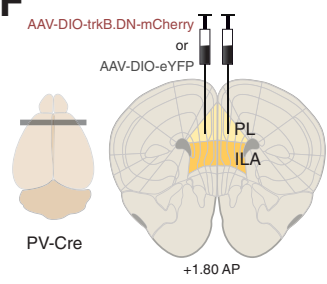

G eYFP: PV-Cre mice injected trkB.DN: PV-Cre mice injected with $\mathbf{H}$

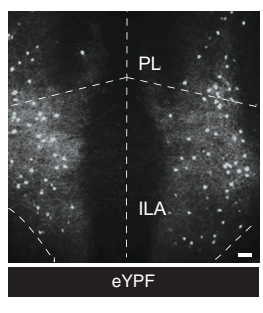
AAV-DIO-trkB.DN-mCherry
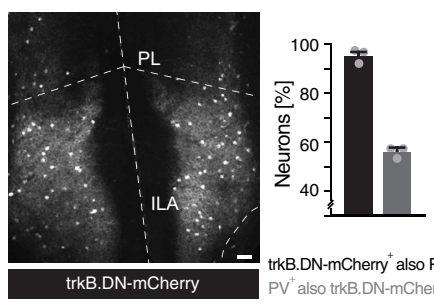

J
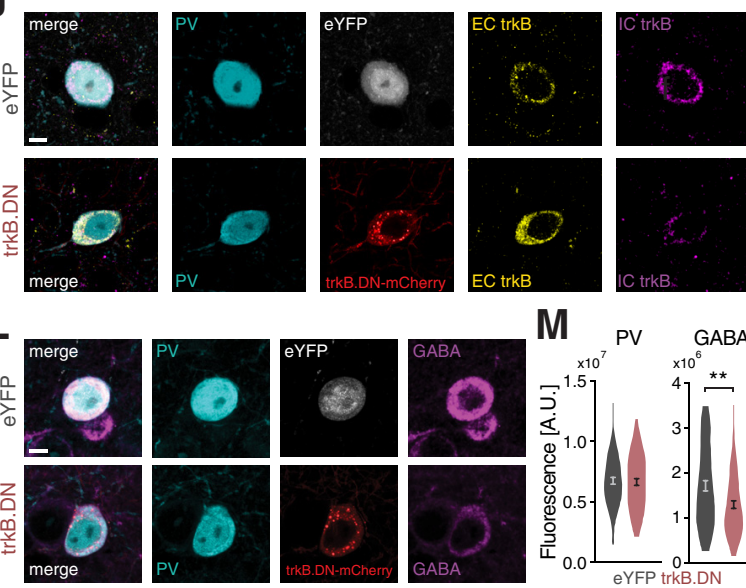

M

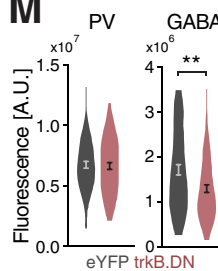

Figure 1. Targeting of trkB.DN to mPFC PV interneurons in adult mice. $A$, Visualization of the molecular diversity of $m P F C$ GABAergic (Vgat-expressing) neurons in a t-SNE plot. Each dot represents an individual GABAergic neuron nucleus $(n=648$ nuclei). $\boldsymbol{B}$, Mapping of the distribution of the expression of Pvalb identified two clusters, cluster 14 ( $n=175$ nuclei) and 15 ( $n=12$ nuclei), expressing high levels of Pvalb. C, Distribution of the expression of Ntrk2 in the Pvalb-expressing clusters 14 and 15; $98.9 \%(173 / 175)$ of the nuclei in clusters 14 , and $100 \%(12 / 12)$ of the nuclei in cluster 15, express Ntrk2. D, Distribution of the expression Bdnf in the Pvalb-expressing clusters 14 and 15;2.9\% (5/175) of the nuclei in cluster 14, and $0 \%(0 / 12)$ of the nuclei in cluster 15 , express Bdnf. $\boldsymbol{E}$, Cre-expressing neurons transduced by AAV-DI0-trkB.DN-mCherry express the virally expressed trkB.DN-mCherry, and the endogenous full-length (trkB.FL) and truncated (trkB.T) trkB receptors. F, AAV-DI0-trkB.DN-mCherry or AAV-DI0-eYFP was injected into the mPFC in adult (8- to 12-week-old) PV-Cre mice, with the injection center targeted to the prelimbic area (PL). G, Bilateral eYFP (left), and trkB.DN-mCherry (right), expression in mPFC neurons in PV-Cre mice four weeks after viral injections. $\boldsymbol{H}$, Specificity and efficiency of targeting of trkB.DN to mPFC PV interneurons at the injection site ( $n=3$ trkB.DN mice). Black bar: percent trkB.DN-mCherry expressing neurons also expressing PV (95.7 $\pm 3.1 \%, 1849 / 1940$ neurons); gray bar: percent mPFC PV expressing neurons also expressing trkB.DNmCherry (56.8 $\pm 4.1 \%$, 1849/3246 neurons). I, Representative immunohistochemical detection of co-expression of PV (green) and trkB.DN-mCherry (red) in the mPFC of a PV-Cre mouse injected with AAV-DI0-trkB.DN-mCherry. J, Representative immunohistochemical detection of PV (cyan), extracellular (EC) trkB domain (yellow), intracellular (IC) trkB domain (pink), and eYFP (white), or trkB.DN-mCherry (red) in a mPFC PV interneuron in an eYFP mouse (top row) or a trkB.DN mouse (bottom row). $\boldsymbol{K}$, Levels of the EC trkB domain (left), the IC domain (middle), and the IC/EC ratio in mPFC PV interneurons expressing trkB.DN-mCherry (red; $n=99$ neurons in 5 trkB.DN mice) or eYFP (gray; $n=74$ neurons in 4 eYFP mice), respectively. EC domain: eYFP: 1,613,709 \pm 85,857 a.u.; trkB.DN-mCherry: 2,571,727 $\pm 145,054$ a.u.; $U=1971, p<0.0001$. IC domain: eYFP: 1,522,892 \pm 191,982 a.u.; trkB.DN-mcherry 675,638 $\pm 103,170$ a.u.; $U=2268, p<0.0001$. IC/EC ratio: eYFP: $0.62 \pm 0.04$; trkB.DN-mcherry $0.25 \pm 0.03 ; U=1455, p<0.0001$. $L$, Representative immunohistochemical detection of PV (cyan), GABA (pink), and eYFP (white) or trkB.DN-mCherry (red), in a mPFC PV interneuron in an eYFP mouse (top row), and a trkB.DN mouse (bottom row). $M$, left, The levels of PV protein in the cell body of mPFC PV interneurons expressing eYFP (gray; 6,757,864 $\pm 276,743$ a.u.; $n=62$ neurons from 3 eYFP mice) or trkB.DN-mCherry (red; $6,641,059 \pm 284,145$ a.u.; $n=68$ neurons from 3 trkB.DN mice), $t=0.2935, p=0.7696$. Right, The levels of GABA in $\mathrm{mPFC}$ PV interneurons expressing eYFP (gray; 1,722,901 $\pm 116,046$ a.u.) and trkB.DN-mCherry (red; 1,298,824 \pm 85,478 a.u.), $\boldsymbol{U}=1539, p=0.0077$. For $\boldsymbol{A}-\boldsymbol{D}$, normalized $\log 2$-expression. For $\boldsymbol{H}, \boldsymbol{K}, \boldsymbol{M}$, data shown as mean $\pm \mathrm{SEM}$. Unpaired test, Twotailed was used to assess significance if data passed the D'Agostino and Pearson normality test, if not, the Wilcoxon rank-
DN can compete for BDNF, but binding of BDNF to trkB.DN does not activate intracellular pathways. Further, as trkB.DN lacks any intracellular trkB domain, its expression does not induce BDNF independent functions conveyed by intracellular domains of truncated trkB isoforms (Fenner, 2012). For adult expression of trkB.DN in mPFC PV interneurons, the AAV was injected bilaterally into the mPFC of 8- to 12-week-old PV-Cre mice (Fig. $1 F$ ). Control mice were generated by injection of an AAV with Cre-dependent expression of eYFP into PV-Cre mice (Fig. $1 F$ ). Throughout the current study, mice injected with AAV-DIO-trkB.DNmCherry are referred to as trkB.DN mice, and mice injected with AAV-DIO-eYFP referred to as eYFP mice. Four weeks later, robust fluorescence was detected in prefrontal neurons in both groups of mice (Fig. 1G). Immunohistochemistry verified specific targeting of trkB.DN to mPFC PV interneurons (95.7 $\pm 3.1 \%$; 1849/1940 trkB.DN-mCherry-expressing neurons also expressing PV; $n=3$ trkB. DN mice; Fig. $1 H, I)$. PV is a calciumbinding protein, with activity regulated expression, and the non-complete coexpression of PV and trkB.DN-mCherry is plausibly explained by PV expression below the detection threshold in a small subset of the PV interneurons (Sohal et al., 2009). The injected volume transduced $56.8 \pm 4.1 \%(1849 / 3246)$ of the $\mathrm{PV}$ interneurons at the injection site (Fig. 1H,I).

To confirm the dominant-negative action of trkB.DN, we used immunohistochemistry to detect the extracellular domain of trkB in the cell bodies of virustransduced PV interneurons in trkB.DN $(n=5)$ and eYFP $(n=4)$ mice (Fig. $1 J, K)$. PV interneurons expressing trkB.DN expressed significantly higher levels of the extracellular trkB domain than PV interneurons expressing eYFP (Fig. $1 K$ ), indicating an overall higher level of trkB in mPFC PV interneurons transduced by AAV-DIO-trkB.DN-mCherry. As mentioned, trkB.DN lacks the intracellular trkB domain, and we therefore next investigated the levels of the intracellular domain and found that PV interneurons expressing trkB.DN expressed significantly

$\leftarrow$

sum test was used. Statistics and source data can be found in Extended Data Figures 1-1, 1-2, respectively; ${ }^{* *} p<0.01$, ${ }^{* * *} p<0.001$. Scale bars: $75 \mu \mathrm{m}(G), 100 \mu \mathrm{m}(\boldsymbol{I}), 10 \mu \mathrm{m}$ (J), $10 \mu \mathrm{m}(L)$. 
A
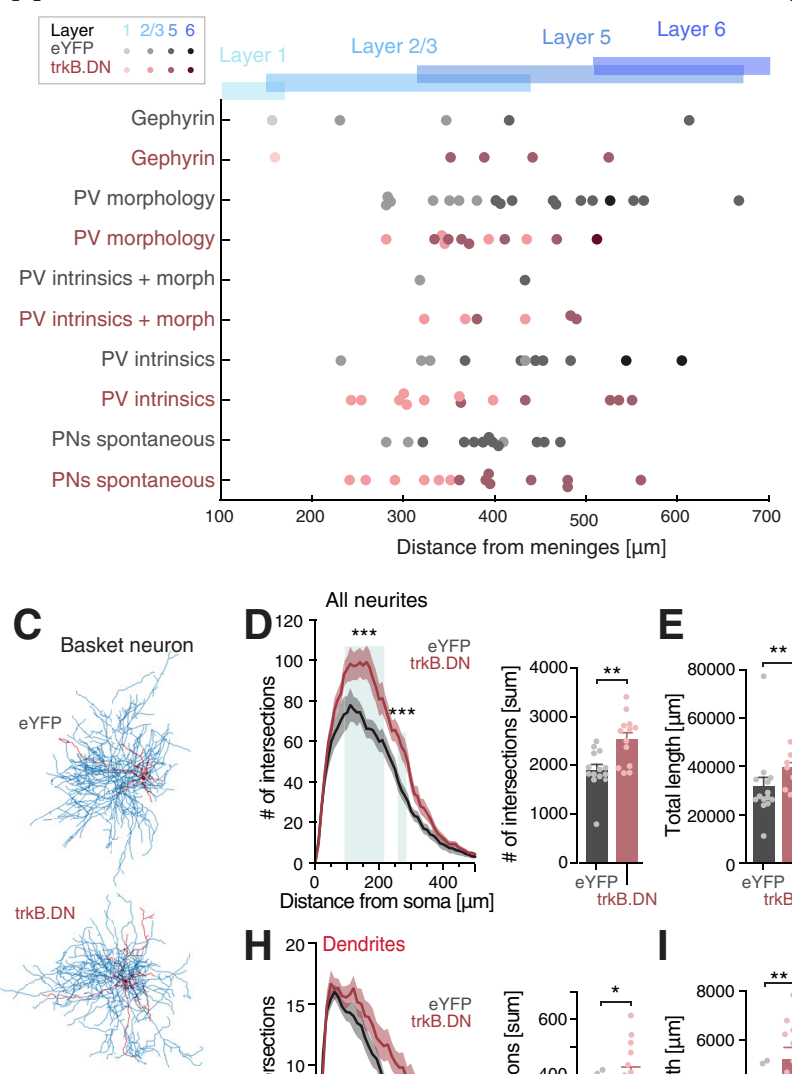

axons
dendrites

Chandelier neuron
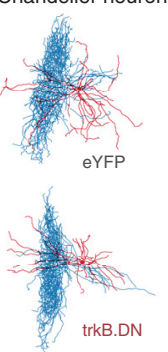
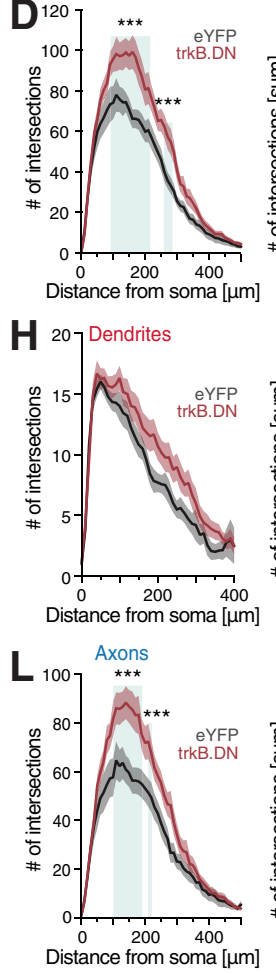

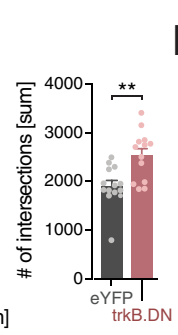

E

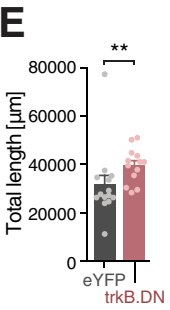

I
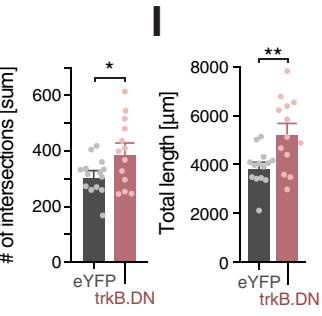

J

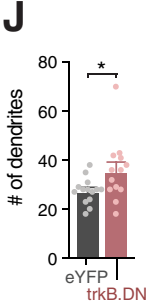

M

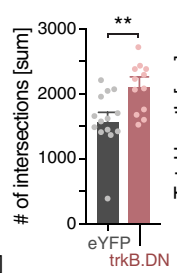

$\mathbf{F}$

N

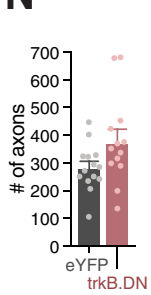

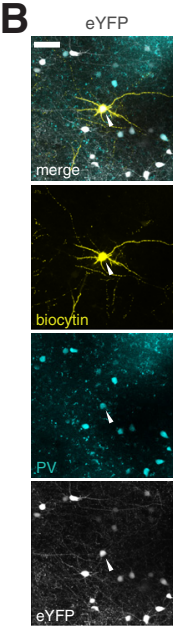

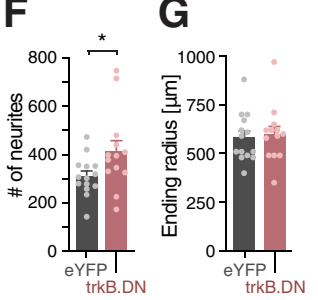

$\mathrm{K}$

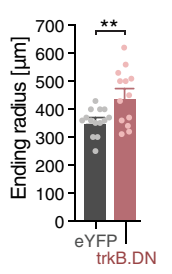

trkB.DN

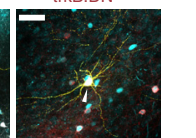

te

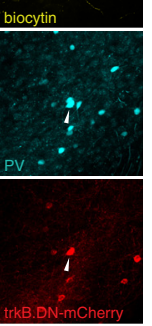

G
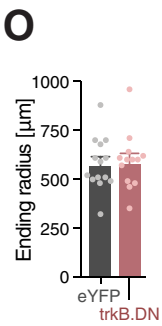

Figure 2. Morphologic alterations in $\mathrm{mPFC}$ PV interneurons expressing trkB.DN. A, Anatomical location of the investigated mPFC neurons. Neurons (dots; eYFP mice: gray shades; trkB.DN mice: red shades) were sampled in different layers across the $\mathrm{mPFC}(+2.2-0.8 \mathrm{~mm}$ AP relative to bregma). The layers vary in thickness across the AP extent, and the horizontal bars in blue shades outline the layers' ML extent. Gephyrin: PV interneurons: $n=4$, in 2 eYFP mice; $n=5$, in 3 trkB.DN mice. PV morphology: biocytin-filled PV basket neurons used for Sholl analysis: $n=14$, in 8 eYFP mice; $n=13$, in 6 trkB.DN mice. PV intrinsics + morph: biocytin-filled PV basket neurons subjected to Sholl analysis and electrophysiology: $n=2$, in 2 eYFP mice; $n=6$, in 3 trkB.DN mice. PV intrinsics: PV interneurons subjected to electrophysiology: $n=38$, in 17 eYFP mice; $n=36$, in 14 trkB.DN mice. PNs spontaneous: PNs subjected to electrophysiology: $n=15$, in 3 eYFP mice; $n=14$, in 3 trkB. DN mice. $\boldsymbol{B}$, Representative biocytin-filled mPFC PV basket neurons stained for biocytin (yellow), PV (cyan), and eYFP (white) or mCherry (red) in an eYFP mouse (left column) and a trkB.DN mouse (right column). C, Representative reconstructions of biocytin-filled mPFC PV basket neurons (top) and chandelier neurons (bottom), with color-coding of the traced dendrites (red) and axons (blue), in an eYFP mouse and a trkB.DN mouse. $\mathbf{D}-\mathbf{0}$, Data based on Sholl analysis of the biocytin-filled mPFC PV basket neurons (eYFP mice: $n=14$; trkB.DN mice: $n=13$ ). $\boldsymbol{D}$, The neurites (dendrites + axons) of PV basket neurons expressing trkB.DN (red) display increased complexity compared with PV basket neurons expressing eYFP (gray), with a significantly increased number of intersections, particularly $100-200 \mu \mathrm{m}$ from the soma, and a significantly increased total number of intersections. eYFP: $1908 \pm 109.9$; trkB.DN: $2536 \pm 140.8 ; U=26, p=0.001$. Blue shading: significant difference between trkB.DN and eYFP mice. $\boldsymbol{E}$, PV basket neurons expressing trkB.DN have significantly increased total neurite length compared with PV basket neurons expressing eYFP. eYFP: 31,554 $\pm 3922 \mu \mathrm{m}$; trkB.DN: $39,614 \pm 2002 \mu \mathrm{m} ; U=27$, $p=0.0013$. $F$, PV basket neurons expressing trkB.DN have significantly increased number of neurites compared with PV basket neurons expressing eYFP. eYFP mice: $310.9 \pm 21.58$ paths; trkB.DN mice: $413.5 \pm 45.49$ paths; $t=2.085, p=0.0474$. G, The ending radius of the neurites does not differ between PV basket neurons expressing trkB.DN and PV basket neurons

lower levels of the intracellular trkB domain than PV interneurons expressing eYFP, reflecting a lower level of trkB.FL in the presence of virally expressed trkB.DN (Fig. $1 K$ ). Concordantly, the intracellular/ extracellular ratio was found to be significantly lower in mPFC PV interneurons expressing trkB.DN than in their eYFP expressing counterparts (Fig. $1 K$ ), and together these experiments demonstrate that trkB.DN acts as a dominant-negative receptor. Immunohistochemistry revealed that adult expression of trkB.DN did not affect the levels of PV, but significantly reduced the levels of GABA in the cell bodies of mPFC PV interneurons (Fig. $1 L, M$ ). This contrasts with findings of reduced PV levels in models of postnatal deletion of trkB, which affect the maturation of the PV neurons (Zheng et al., 2011).

\section{Morphologic alterations of mPFC PV interneurons}

For a detailed study of cell morphologies and physiology, acute brain slices were

expressing eYFP. eYFP mice: $582.1 \pm 33.53 \mu \mathrm{m}$; trkB.DN mice: $598.5 \pm 40.19 \mu \mathrm{m} ; U=83.5, p=0.7281$ for all neurites combined. $\boldsymbol{H}$, The dendrites of PV basket neurons expressing trkB.DN display increased complexity compared with PV basket neurons expressing eYFP, as observed by an increased number of intersections at most distances from the soma and a significantly increased total number of intersections. eYFP: $312.1 \pm 17.15 ; \quad$ trkB.DN: $395.8 \pm 33.4$; $t=2.278, p=0.0316$. $I-K$, Comparison of the total dendritic length ( $\boldsymbol{I}$; eYFP mice: $3896 \pm 199.8 \mu \mathrm{m}$; trkB.DN mice: $5290 \pm 405 \mu \mathrm{m} ; t=3.156, p=0.0041$ ), number of dendrites ( $\boldsymbol{J}$; eYFP mice: $27.43 \pm 1.41$ dendrites; trkB.DN mice: $35.69 \pm 3.54$ dendrites; $U=45 p=0.0236)$, and ending radius of the dendrites ( $\boldsymbol{K}$; eYFP mice: $357.1 \pm 12.69 \mu \mathrm{m}$; trkB.DN mice: $446.2 \pm 27.65 \mu \mathrm{m} ; t=2.998, p=0.0061) . L$, The axons of PV basket neurons expressing trkB.DN display increased complexity compared with PV basket neurons expressing eYFP, with a significantly increased number of intersections, particularly $100-200 \mu \mathrm{m}$ from the soma, and a significantly increased total number of intersections. eYFP: $1601 \pm 118.8$; trkB.DN: $2153 \pm$ $114.2 ; U=27, p=0.0013$. Blue shading: significant difference between trkB.DN and eYFP mice. $\boldsymbol{M}-\mathbf{0}$, Comparison of the total axonal length $(\boldsymbol{M}$; eYFP mice: $24,863 \pm 1932 \mu \mathrm{m}$; trkB.DN mice: $34,337 \pm 1705 \mu \mathrm{m}$; $U=20, p=0.0002)$, number of axons ( $\boldsymbol{N}$, eYFP mice: $283.5 \pm 22.2$ axons; trkB.DN mice: $377.8 \pm 43.95$ axons; $t=1.958, p=0.0614)$, and ending radius of the axons (0; eYFP mice: $578.6 \pm 36.02 \mu \mathrm{m}$; trkB.DN mice: $591.5 \pm 40.22 \mu \mathrm{m} ; U=89, p=0.9334)$. Data shown as mean \pm SEM. Unpaired $t$ test, Two-tailed was used to assess significance if data passed the D'Agostino and Pearson normality test, if not, the Wilcoxon rank-sum test was used. For Sholl analysis plot $(\boldsymbol{D}, \boldsymbol{H}, \boldsymbol{L})$, significance was tested with Multiple $t$ tests with the HolmSidak method. Source data can be found in Extended Data Figure 2-1. Scale bar: $50 \mu \mathrm{m}(\boldsymbol{B}) ;{ }^{*} p<0.05$, ${ }^{* *} p<0.01,{ }^{* * *} p<0.001$. 
A

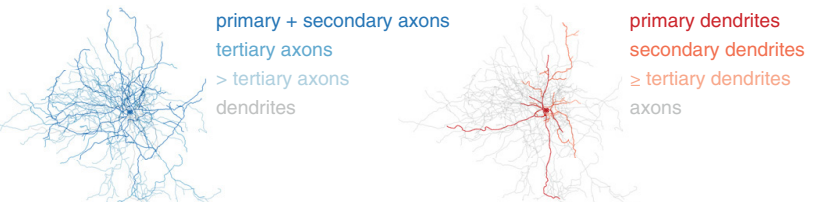

B
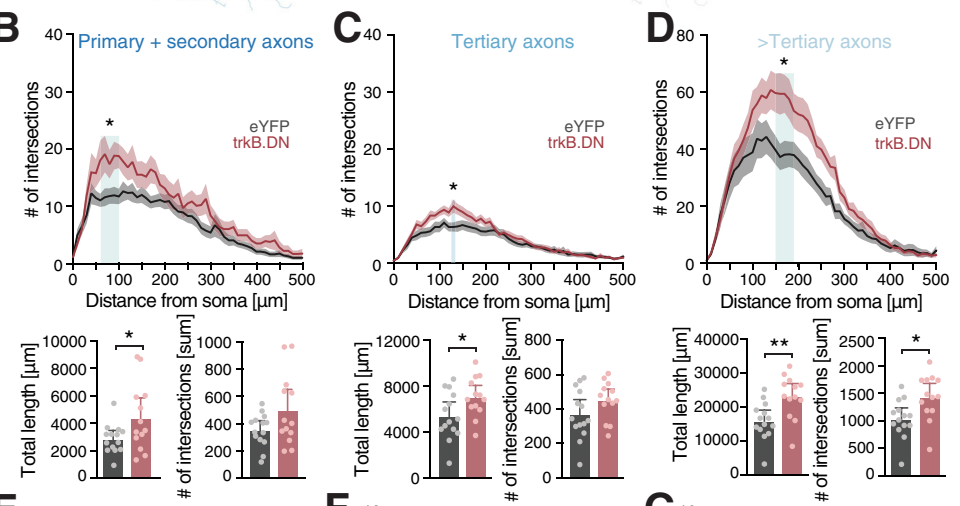

E
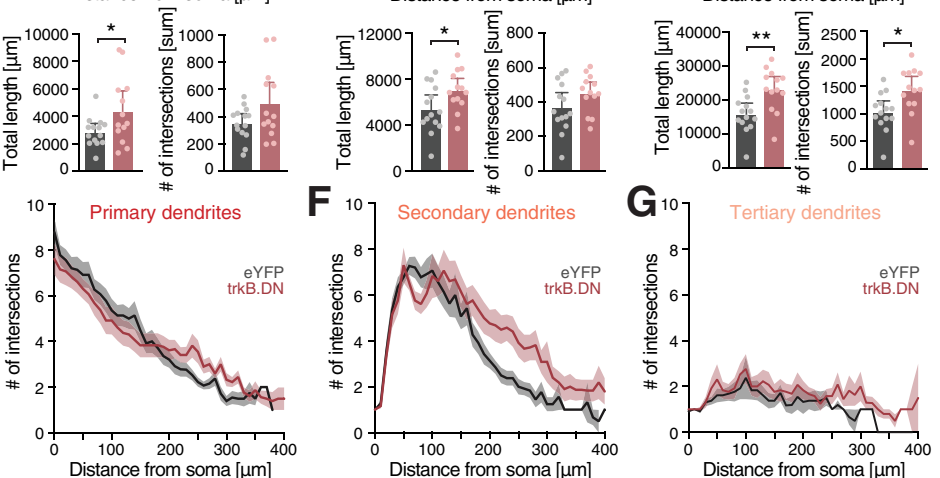

\section{.}
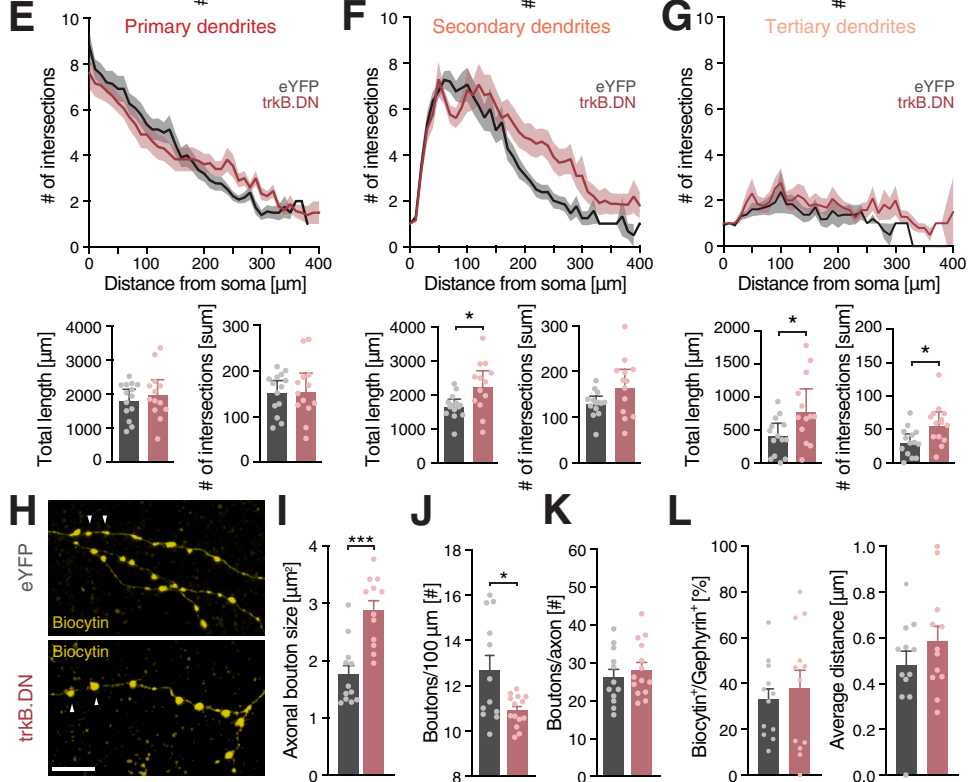

J

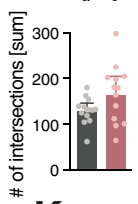

K

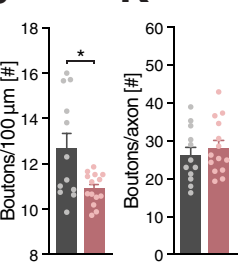

N trkB.DN
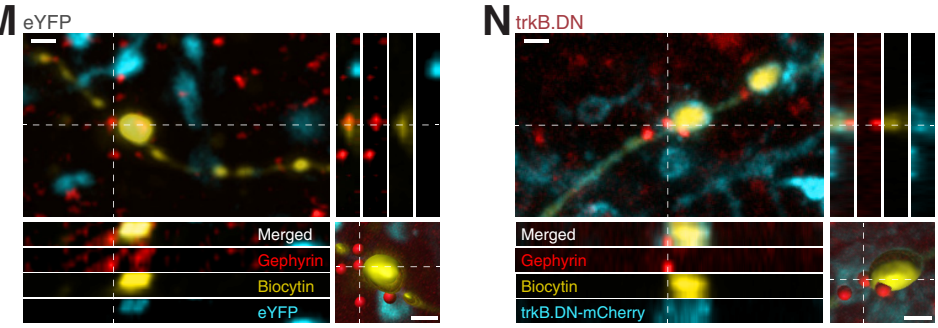

Figure 3. Detailed morphologic analysis of $\mathrm{MPFC} P V$ interneurons expressing trkB.DN. $\boldsymbol{A}$, Reconstructed biocytin-filled mPFC PV basket neuron with color coding of the axonal (left) and dendritic (right) hierarchical branching. Primary neurites (axons and dendrites): extending from the cell body; secondary neurites: extending from primary neurites; tertiary neurites: extending from secondary neurites, and so on. $\boldsymbol{B}-\boldsymbol{G}$, Data based on Sholl analysis of biocytin-filled mPFC PV basket neurons. eYFP: $n=14$ neurons in 8 mice; trkB.DN: $n=13$ neurons in 6 mice. $\boldsymbol{B}-\boldsymbol{D}$, Sholl profile, the total length, and total number of intersections for the axons. Blue shading: significant difference between trkB.DN and eYFP mice. $\boldsymbol{B}$, Primary and secondary axons: total length (eYFP: $2878 \pm 284.7 \mu \mathrm{m}$; trkB.DN: $4377 \pm 675.6 \mu \mathrm{m} ; t=2.099, p=0.0461)$ and total number of intersections (eYFP: $354 \pm 32.17$; trkB.DN: $494.8 \pm 72.16 ; t=1.828, p=0.0795$ ). $C$, Tertiary axons: total length (eYFP: $5421 \pm 554.3 \mu \mathrm{m}$; trkB.DN: $7042 \pm 479.3 \mu \mathrm{m} ; t=2.197, p=0.0375$ ) and total number of intersections (eYFP: $371.0 \pm 38.7$; trkB.DN: $450.4 \pm 30.6 ; t=1.591, p=0.1241$ ). $\boldsymbol{D}$, $>$ Tertiary axons: total length (eYFP: $16,030 \pm 1450 \mu \mathrm{m}$; trkB.DN: $23,184 \pm 1745 \mu \mathrm{m} ; t=3.171$, $p=0.0040$ ) and total number of intersections (eYFP: $1041 \pm 91.4$; trkB.DN: $1436 \pm 114.2 ; t=2.715$, $p=0.0118)$. $\boldsymbol{E}-\boldsymbol{G}$, Sholl profile, the total length, and total number of intersections for the dendrites. $\boldsymbol{E}$, Primary dendrites: total length (left; eYFP: $1851 \pm 139.6 \mu \mathrm{m}$; trkB.DN: $2003 \pm 200.5 \mu \mathrm{m} ; t=0.6321$, prepared from cohorts of trkB.DN $(n=14)$, and eYFP $(n=18)$ mice. Detected fluorescent PV neurons were filled with biocytin and subjected to slice electrophysiology and/or detailed morphologic analyses (Fig. 2A). The PV identity was confirmed in all biocytin-filled neurons, and the reconstructions identified PV basket neurons and a minor population of chandelier neurons with a strikingly differential morphology (Fig. 2B,C). Because of the low sampling of chandelier neurons (trkB.DN mice: $n=4$; eYFP mice: $n=7$ ) only PV basket neurons (trkB.DN mice: $n=13$; eYFP mice: $n=14$ ) were included in our further morphologic investigations. 3D morphometric analysis (Sholl analysis) of the biocytin-labeled neurons revealed a significantly increased neurite (axons + dendrites) arborization in mPFC PV basket neurons expressing trkB.DN, particularly $100-200 \mu \mathrm{m}$ from the soma (Fig. 2D). In addition, the total length and the number of neurites were significantly increased (Fig. $2 E, F$ ). However, the ending radius of the neurites was unchanged, indicating that the overall size of the PV interneurons expressing trkB.DN was unaltered (Fig. 2G). Both dendrites and axons displayed increased arborization and total length

$p=0.5331$ ) and total number of intersections (right; eYFP: $153.9 \pm 11.8$; trkB.DN: $157.6 \pm 17.5 ; t=0.1768, p=0.8611) . F$, Secondary dendrites: total length (eYFP: $1663 \pm 95.9 \mu \mathrm{m}$; trkB.DN: $2252 \pm 206.5 \mu \mathrm{m} ; t=2.648$, $p=0.0138$ ) and total number of intersections (eYFP: $130.1 \pm 7.5$; trkB.DN: $166.7 \pm 17.5 ; t=1.972, p=0.0598)$. $\mathbf{G}$, Tertiary dendrites: total length (eYFP: $407.6 \pm 75.0 \mu \mathrm{m}$; trkB.DN: $811.2 \pm 141.8 \mu \mathrm{m} ; t=2.569, p=0.0166$ ) and total number of intersections (eYFP: $31.6 \pm 5.6$; trkB.DN: $58.1 \pm 8.6$; $t=2.620, p=0.0147$ ). $\boldsymbol{H}$, Representative images of axonal boutons (arrowheads) in biocytin-filled mPFC PV basket neurons expressing eYFP (top) or trkB.DN (bottom). $I$, Expression of trkB.DN results in significantly increased mean bouton size. eYFP: $1.76 \pm 0.16 \mu \mathrm{m}^{2}, n=12$ axonal paths from 4 mice; trkB.DN: $2.88 \pm 0.17 \mu \mathrm{m}^{2}, n=12$ axonal paths from 4 mice; $t=4.806$, $p<0.0001$. $\boldsymbol{J}, \boldsymbol{K}$, Quantification of axonal boutons in biocytin-filled mPFC PV basket neurons expressing trkB.DN ( $n=14$ neurons in 6 trkB.DN mice) or eYFP ( $n=12$ neurons in 8 eYFP mice). $J$, Expression of trkB.DN results in significantly decreased number of boutons $/ 100 \mu \mathrm{m}$. eYFP: $12.69 \pm 0.66$ boutons $/ 100 \mu \mathrm{m}$; trkB.DN: $10.92 \pm 0.18$ boutons $/ 100 \mu \mathrm{m} ; t=2.782, p=0.0104$. $\boldsymbol{K}$, The total number of boutons along the entire axonal path does not differ between biocytin-filled mPFC PV basket neurons expressing trkB.DN or eYFP. eYFP: $26.19 \pm 2.1$ boutons/axon, trkB.DN: $28.26 \pm 1.93$ boutons/axon; $t=0.7255, p=0.4752$. L, Quantification of gephyrin labeled postsynaptic structures in the immediate vicinity of biocytin labeled boutons in biocytinfilled mPFC PV basket neurons expressing trkB.DN ( $n=12$ axonal paths, in 3 trkB.DN mice) or eYFP ( $n=12$ axonal paths, in 2 eYFP mice). Left, Percentage of biocytin labeled boutons with gephyrin spots in the immediate vicinity. eYFP mice: $32.9 \pm 4.8 \%$; trkB.DN mice: $38.0 \pm 7.9 \% ; t=0.5456, p=0.5908$. Right, Mean center-to-center distances between neighboring gephyrin spots and biocytin labeled boutons. eYFP: $0.48 \pm 0.06 \mu \mathrm{m}$; trkB.DN: $0.58 \pm 0.06 \mu \mathrm{m} ; t=1.140, p=0.2667$. $\boldsymbol{M}, \mathbf{N}$, Representative immunohistochemical detection of gephyrin (red), biocytin (yellow) in MPFC PV interneurons expressing eYFP ( $\boldsymbol{M}$; cyan) or trkB.DN ( $\boldsymbol{N}$; cyan), with orthogonal views in the $z$-plane. Crosshairs highlight examples of gephyrin spots in the vicinity of a biocytin-filled axonal bouton. 3D examples of gephyrin labeling detected as spots near reconstructed biocytin-filled axonal for both $\mathrm{MPFC} P \mathrm{PV}$ interneurons expressing eYFP ( $\boldsymbol{M}$; bottom-right) or trkB.DN ( $\boldsymbol{N}$; bottom-right). Data shown as mean \pm SEM. For Sholl analysis plot $(\boldsymbol{B}-\boldsymbol{G})$, significance was tested with multiple $t$ tests with the Holm-Sidak method. For bar plots, unpaired $t$ test, two-tailed was used to assess significance if data passed the D'Agostino and Pearson normality test, if not, Wilcoxon rank-sum test was used. Source data can be found in Extended Data Figure 3-1. Scale bars: $10 \mu \mathrm{m}(\boldsymbol{H})$ and $1 \mu \mathrm{m}$ $(M, N) ;{ }^{*} p<0.05,{ }^{* *} p<0.01,{ }^{* * *} p<0.001$. 
A

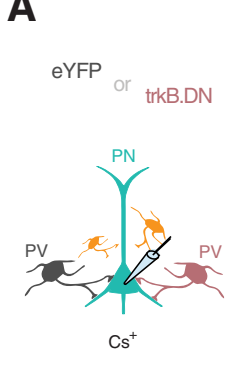

B

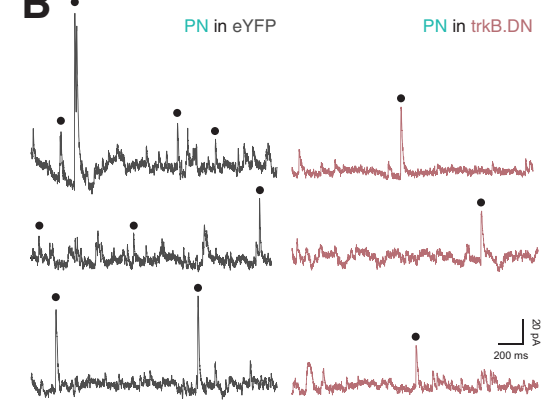

\section{C}

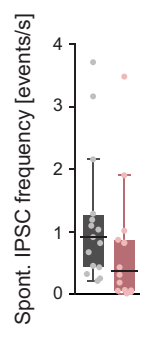

D

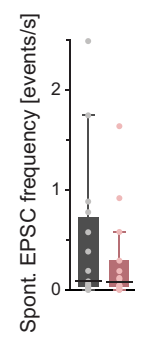

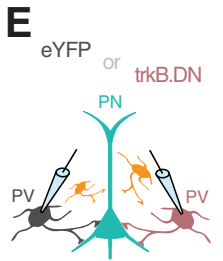

low $\left[\mathrm{Cl}^{-}\right]$

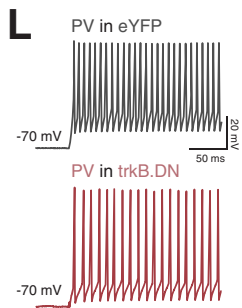

$\mathbf{Q}$

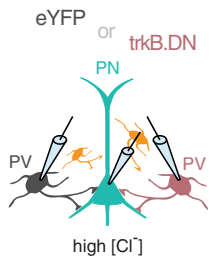

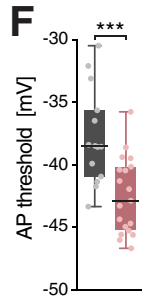
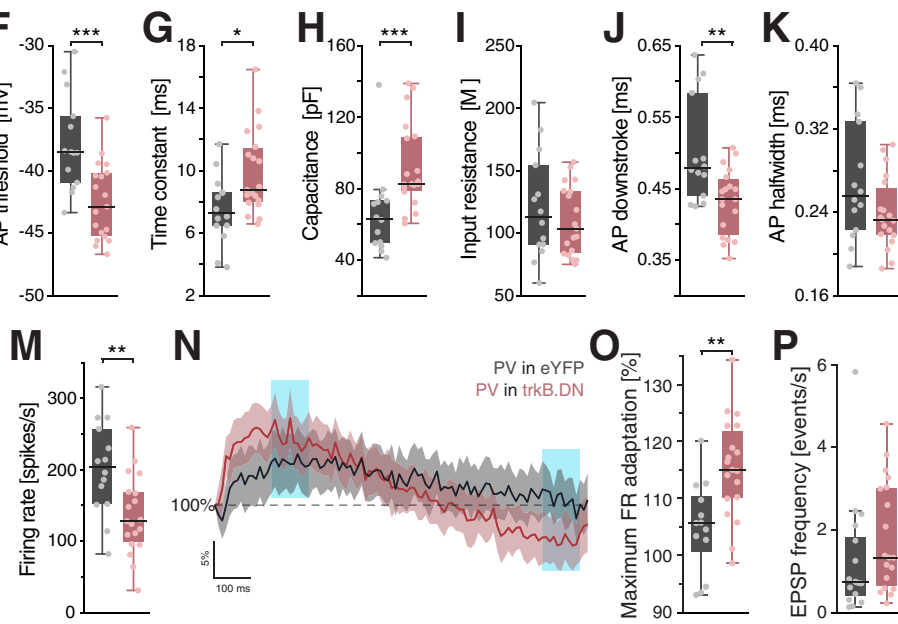

N
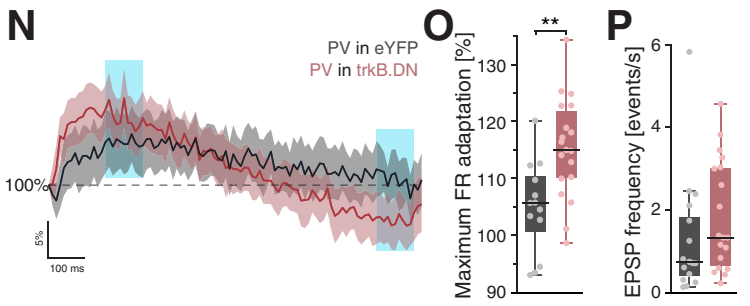
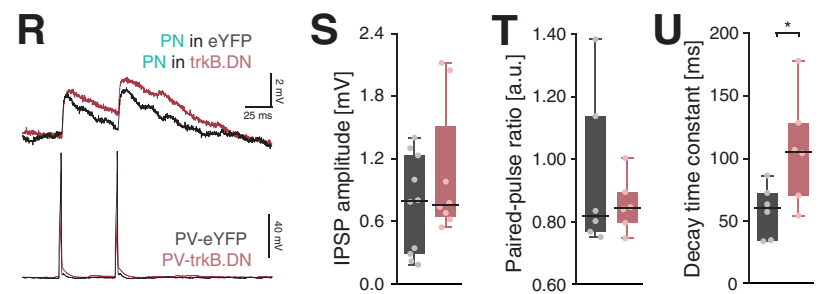

Figure 4. Alterations in intrinsic properties and synaptic connectivity of mPFC PV interneurons expressing trkB.DN. $\boldsymbol{A}-\boldsymbol{E}$, Voltage clamp recordings of spontaneous events in mPFC PNs in eYFP (gray) and trkB.DN mice (red). $\boldsymbol{A}$, Schematic of PN recordings. $\mathrm{CS}^{+}$: cesium. $\boldsymbol{B}$, Representative traces of IPSCS (black dots) in PNs recorded at $0 \mathrm{mV}$ holding potential. $\mathbf{C}, \boldsymbol{D}$, Synaptic events in PNs in eYFP (gray; $n=15$ PNs, in 3 mice) and trkB.DN mice (red; $n=14$ PNs, in 3 mice), respectively. $C$, The frequency of IPSCs was considerably reduced in PNs in trkB.DN mice compared with in eYFP mice. eYFP: 0.91 events/s; trkB.DN: 0.37 events $/ s ; U=157.5, p=0.0521$. D, The frequency of EPSCs (recorded at $-70 \mathrm{mV}$ ) in PNs was very low and did not differ between eYFP and trkB.DN mice. eYFP: 0.08 events/s; trkB.DN: 0.08 events $/ s ; U=124.5, p=0.6153$. $\boldsymbol{E}-\boldsymbol{P}$, Current clamp recordings of mPFC PV interneurons expressing eYFP (eYFP mice; gray) or trkB.DN (trkB.DN mice; red). $E$, Schematic of PV recordings. $\mathrm{Cl}^{-}$: chloride. $\boldsymbol{F}-\boldsymbol{K}$, Intrinsic properties of mPFC PV interneurons expressing eYFP $(n=14$, in 10 mice) or trkB.DN ( $n=19$, in 6 mice). $\boldsymbol{F}$, The AP threshold was significantly reduced in PV interneurons in trkB.DN mice compared with in eYPF mice. eYFP: $-38.53 \mathrm{mV}$; trkB.DN: $-42.88 \mathrm{mV} ; t=3.9113, p=0.0005$. G, The membrane time constant was significantly increased in PV interneurons in trkB.DN mice compared with in eYPF mice. eYFP: $7.30 \mathrm{~ms}$; trkB.DN: $8.76 \mathrm{~ms}$; $t=-2.6968, p=0.01$. $\boldsymbol{H}$, The membrane capacitance was significantly increased in PV interneurons in trkB.DN mice compared with in eYPF mice. eYFP: 82.6 pF; trkB.DN: $63.1 \mathrm{pF} ; U=41, p=0.0009$. $I$, The input resistance in PV interneurons did not differ between eYFP and trkB.DN mice. eYFP: $112.81 \mathrm{M} \Omega$; trkB.DN: $103.68 \mathrm{M} \Omega ; t=0.8877, p=0.4$. J, The AP downstroke duration was significantly reduced in PV interneurons in trkB.DN mice compared with in eYPF mice. eYFP: $0.4785 \mathrm{~ms}$; trkB.DN: $0.4350 \mathrm{~ms} ; t=3.3914, p=0.0019$. $\boldsymbol{K}$, The AP halfwidth (width at half maximum) in PV interneurons did not differ between eYFP and trkB.DN mice. eYFP: $0.2560 \mathrm{~ms}$; trkB.DN: $0.2330 \mathrm{~ms} ; t=1.9006, p=0.07$. L, Representative AP traces at twice the rheobase current. $\boldsymbol{M}$, The firing rate during $1 \mathrm{~s}$ at twice the rheobase current injection was significantly reduced in PV interneurons in trkB.DN mice compared with in eYPF mice. eYFP: 204 spikes/s $(n=14$, in 10 mice); trkB.DN: 128 spikes/s ( $n=19$, in 6 mice); $t=3.1393, p=0.004$. $N$, Firing rate adaptation over $1 \mathrm{~s}$ current pulse at twice the rheobase current (eYFP: $n=13 \mathrm{PV}$, in 10 mice; trkB.DN: $n=19 \mathrm{PV}$, in 6 mice). Blue shading: the maximal and minimal immediate firing rate $+/-55 \mathrm{~ms}$ (for details, see Materials and Methods). $\mathbf{0}$, The maximum firing rate adaptation (the ratio between the maximal and minimal immediate firing rate; blue shading in $\boldsymbol{N}$ ), was significantly increased in PV interneurons in trkB.DN mice compared with in eYPF mice. eYFP: 105.7\%; trkB.DN: 115.0\%; $t=-3.3788, p=0.002$. $\boldsymbol{P}$, The frequency of EPSPs (recorded at $-70 \mathrm{mV}$ ) was slightly increased in PV interneurons in trkB.DN mice compared with in eYPF mice. eYFP: 0.74 events/s ( $n=17$, in 11 mice); trkB.DN: 1.33 events/s $(n=19$, in 6 mice); $U=91, p=0.0931$. $\mathbf{Q}-\boldsymbol{U}$, Paired recordings of mPFC presynaptic PV interneurons and postsynaptic PNs (PV-PN pairs) in eYFP (gray) and trkB.DN mice (red). $\mathbf{Q}$, Schematic of the paired in $\mathrm{mPFC}$ PV interneurons expressing trkB.DN (Fig. 2H-O). However, while the number and the ending radius of the dendrites were significantly increased (Fig. 2J, $K$ ), the number of axons was not changed, nor the axonal ending radius (Fig. 2N,O). Instead, the axonal arborization was significantly increased $100-200 \mu \mathrm{m}$ from the soma (Fig. $2 L$ ), affecting primary, secondary, tertiary, and more distal axon branches (Fig. 3A-D). The dendritic changes in trkB.DN-expressing PV neurons affected secondary and tertiary dendrites, distal to the peak of the axonal changes (Fig. $3 E-G$ ). Adult reduction of $\mathrm{BDNF} / \mathrm{trkB}$ signaling, thus, altered both the presynaptic and postsynaptic compartments of prefrontal PV interneurons. To infer how the increased axonal complexity affected the synaptic connections of PV interneurons onto neurons in the local network, we next analyzed the inhibitory synapses of biocytin-filled PV interneurons. The boutons of trkB.DN-expressing $\mathrm{PV}$ interneurons were rounded and corpulent, with significantly increased size, compared with the boutons of eYFP expressing PV interneurons (Fig. 3H,I). Counting of axonal boutons revealed a significantly decreased density of boutons along the axons of trkB.DN-expressing PV interneurons (Fig. 3J). However, accounting for the increased axonal length revealed that the total number of boutons did not differ between trkB.DN and eYFP mice (Fig. $3 K)$. Quantification of the juxtaposition of biocytin-filled axonal boutons with gephyrin, a GABAergic postsynaptic scaffold protein, indicated that although being enlarged, the boutons on trkB.DNexpressing PV interneurons indeed made

$\leftarrow$

recordings. $\boldsymbol{R}$, Representative traces of evoked APs in PV interneurons (bottom) and the corresponding IPSPs in PNs (top). S, The amplitude of the first IPSP in the PNs did not differ between eYFP and trkB.DN mice. eYFP: $0.79 \mathrm{pA}$ (10 pairs, in 8 mice); trkB.DN: $0.76 \mathrm{pA}$, (8 pairs, in 7 mice); $t=$ $-1.1709, p=0.2588$. $T$, The paired-pulse ratio of the PV-PN pairs did not differ between eYFP and trkB.DN mice. eYFP: 0.78 ( $n=6$ pairs, in 5 mice); trkB.DN: 0.81 ( $n=6$ pairs, in 6 mice); $t=0.8238, p=0.4293$. $\boldsymbol{U}$, The decay time constant of the first IPSP in PNs was significantly increased in trkB.DN mice compared with in eYPF mice. eYFP: $60.36 \mathrm{~ms}(n=6$ pairs, in 5 mice); trkB.DN: $105.58 \mathrm{~ms}$ ( $n=6$ pairs, in 6 mice); $t=-2.4738, p=0.0329$. For boxplots $(\boldsymbol{C}, \boldsymbol{D}, \boldsymbol{F}-\boldsymbol{K}, \boldsymbol{M}, \mathbf{0}, \boldsymbol{P}$, $S-U)$, data shown as median, box (25th and 75th percentiles), and whiskers (data points that are not outliers). Reported values represent median. For $\boldsymbol{N}$, data shown as mean and confidence interval. Unpaired $t$ test, two-tailed was used to assess significance if data passed the D'Agostino and Pearson normality test, if not, the Wilcoxon rank-sum test was used. Source data can be found in Extended Data Figure $4-1 ;{ }^{*} p<0.05,{ }^{* *} p<0.01,{ }^{* * *} p<0.001$. 


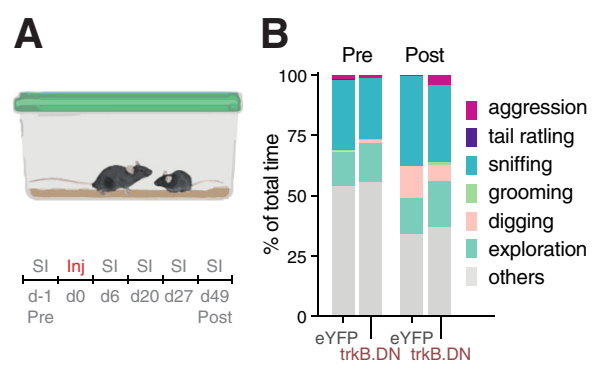

C Aggression

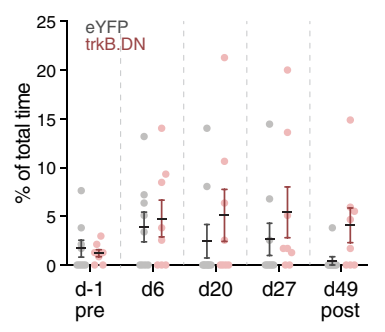

H Exploration

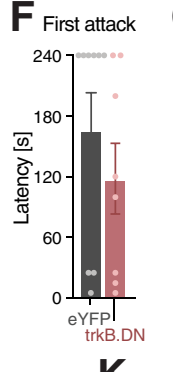

G Snifing

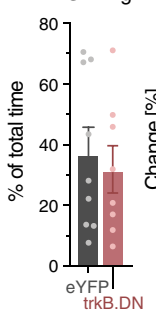

$\mathbf{K}$ Open field

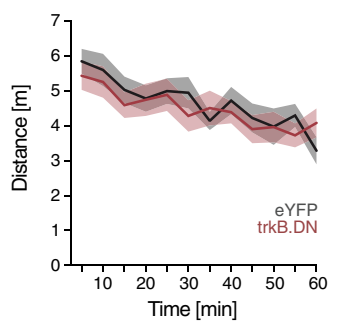

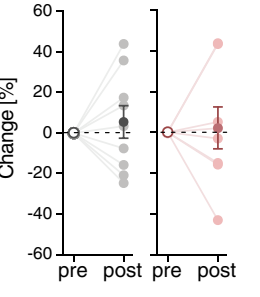

Total distance

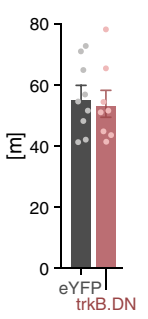

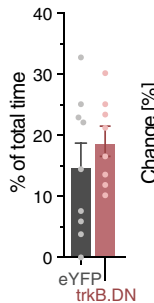

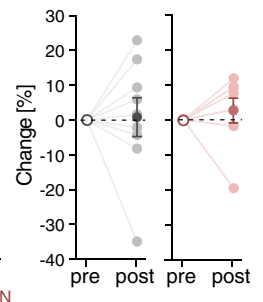

L Center visits

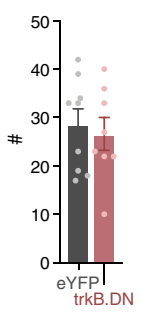

M center velocity

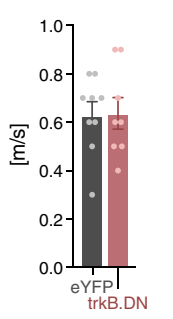

D Aggression

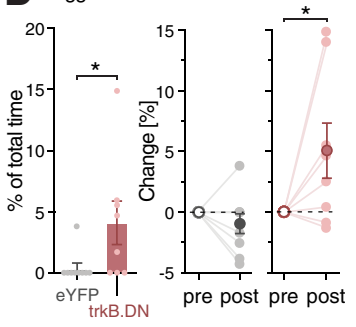

I Grooming

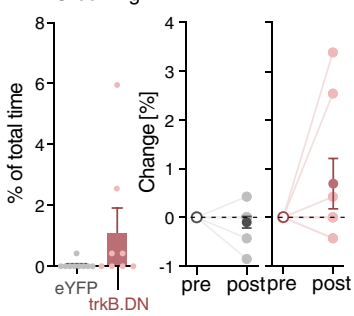

E Aggressive bouts

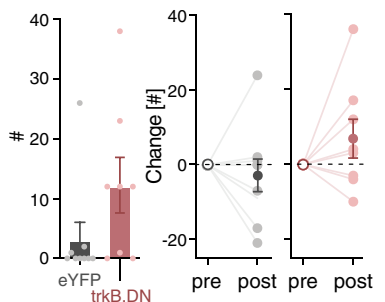

J Digging

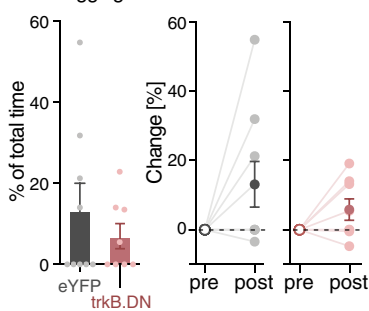

$\mathbf{N}_{\text {Time in centre }}$
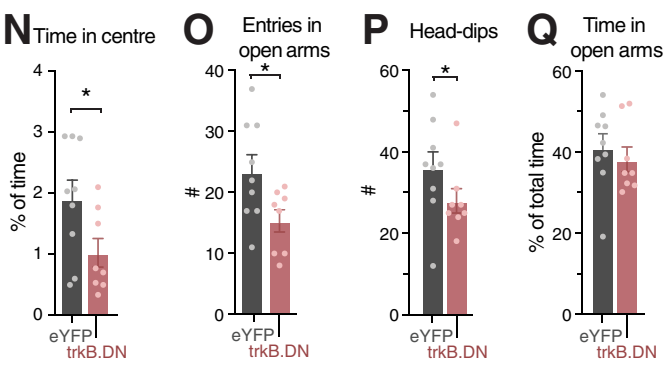

Figure 5. Behavioral alterations in trkB.DN mice. $\boldsymbol{A}-\boldsymbol{J}$, SI investigated by the resident-intruder procedure. trkB.DN mice ( $n=8$ males), eYFP mice ( $n=9$ males). $\boldsymbol{A}$, The resident-intruder procedure was conducted $1 \mathrm{~d}$ before viral injection and four times after injection. Pre $=$ before viral injection, post $=49 \mathrm{~d}$ after injection. On all occasions, the SI lasted 4 min. $\boldsymbol{B}$, Percentage of total time spent in non-social (grooming, digging, cage exploration) and social (aggression, tail rattling, sniffing) behaviors for trkB.DN and eYFP mice during SI, preinjection (d-1), and postinjection (d49). No behavioral differences were observed between trkB.DN and eYFP mice before the viral injections (d-1; pre): aggression (eYFP: $1.70 \pm 0.87 \%$; trkB.DN: $1.22 \pm 0.35 \%$; $t=0.4857$, $p=0.6342$ ), tail rattling (eYFP: $0.33 \pm 0.33 \%$; trkB.DN: $0.16 \pm 0.16 \% ; t=0.4477, p=0.6608$ ), sniffing (eYFP: $29.27 \pm 5.64 \%$; trkB.DN: $25.11 \pm 4.71 \% ; t=0.5583, p=0.5849$ ), grooming (eYFP: $0.14 \pm 0.10 \%$; trkB.DN: $0.48 \pm 0.32 \% ; t=1.068, p=0.3022$ ), digging (eYFP: $0.47 \pm 0.38 \%$; trkB.DN: $1.17 \pm 0.68 \% ; t=0.9228, p=0.3707$ ), exploration (eYFP: $14.09 \pm 4.74 \%$; trkB. DN: $16.17 \pm 2.99 \% ; t=0.3603, p=0.7236$ ), and others (eYFP: $53.99 \pm 6.91 \%$; trkB.DN: $55.69 \pm 6.06 \% ; t=0.1832, p=0.8578)$. $C-\boldsymbol{F}$, Expression of trkB.DN in $\mathrm{mPFC}$ PV interneurons in adult mice results in increased aggression. C, Percentage of time in aggression in the resident intruder procedure $1 \mathrm{~d}$ before viral injection (d-1; pre), and 6, 20, 27, and 49 (d49, post) days after injection for trkB.DN and eYFP mice. $\boldsymbol{D}$, left, Percent time in aggression d49 (post): eYFP: $0.4 \pm 0.4 \%$; trkB.DN: $4.1 \pm 1.7 \%$; $U=16, p=0.0226$. Right, Change (percent of time) in aggression (pre to post): eYFP: $-0.9 \pm 0.8 \% ; W=-8, p=0.1875 ;$ trkB.DN: $5 \pm 2.2 \% ; W=26, p=0.0391$. $\boldsymbol{E}$, left, Number of aggressive bouts d49 (post): eYFP: $3.2 \pm 2.9$ bouts; trkB.DN: $12.25 \pm 4.6$ bouts; $U=18.5, p=0.0771$. Right, Change (number) of aggressive bouts (pre to post): eYFP: $-3.0 \pm 4.3$ bouts; $W=-8, p=0.2891$; trkB.DN: $6.9 \pm 5.2$ bouts; $W=16$, $p=0.1523$. $\boldsymbol{F}$, Latency to first attack d49 (post): eYFP: $166 \pm 37$ s; trkB.DN: $118 \pm 35$; $t=0.9349, p=0.2594$. G-J, Non-aggressive behaviors scored in the resident intruder procedure. (No differences were found between trkB.DN and eYFP mice except for measures of aggression). Left, d49 (post). Right, Change between timepoints pre (d1) and post (d49). $\mathbf{G}$, left, Percent time in sniffing d49 (post): eYFP: $37.1 \pm 8.6 \%$; trkB.DN: $31.9 \pm 7.8 \% ; t=0.4471, p=0.6612$ ). Right, Change (percent of time) in sniffing (pre to post): eYFP: $5.0 \pm 8.2 \% ; W=7, p=0.3672 ;$ trkB. DN: $2.2 \pm 10.5 \% ; W=2, p=0.4727$. $\boldsymbol{H}$, left, Percent time in exploration d49 (post): eYFP: $15 \pm 3.8 \%$; trkB.DN: $19 \pm 2.5 \% ; t=0.8738, p=0.3960$. Right, Change (percent of time) in exploration (pre to post): eYFP: $0.9 \pm 5.6 \%, W=7, p=0.3672 ;$ trkB.DN: $2.9 \pm 3.6 \% ; W=18, p=0.1172$. I, left, Percent time in grooming d49 (post): eYFP: $0.05 \pm 0.05 \%$; trkB.DN: $1.17 \pm 0.749 \% ; U=21, p=0.0905$ ). Right, Change (percent of time) in grooming (pre to post): eYFP: $-0.09 \pm 0.12 \% ; W=-3, p=0.375$ ), trkB.DN: $0.69 \pm 0.51 ; W=6, p=0.2500$. $J$, Left, Percent time in digging d49 (post): eYFP: $13.6 \pm 6.5 \%$; trkB.DN: $7 \pm 3.1 \% ; t=0.8734, p=0.3962$. Right, Change (percent of time) in digging (pre to post): eYFP: $13.10 \pm 6.59 \%$, $W=13, p=0.0625$; trkB.DN: $5.851 \pm 3.038 \% ; W=15, p=0.0781$. $(\boldsymbol{K}-\boldsymbol{N})$ Open field test. Same mice as in $\boldsymbol{A}-\boldsymbol{J}$, performed d68. $\boldsymbol{K}$, No difference was found in locomotion between trkB.DN mice and eYFP mice. Locomotion during $60 \mathrm{~min}$ : eYFP: $55.94 \pm 3.87$; trkB.DN: $53.83 \pm 4.39$ meters; $t=0.3613, p=0.7229$. Bin size: 5 min. $L$, No difference was found in the number of center visits the first $3 \mathrm{~min}$ in the open field test between trkB.DN and eYFP mice: eYFP: $28.7 \pm 3.2$; trkB.DN: $26.6 \pm 3.4$ center visits; $t=0.4403, p=0.666$. $M$, No difference was found in the velocity in the center of the open field box for the first $3 \mathrm{~min}$ of the test: eYFP: $0.63 \pm 0.05$; trkB.DN: $0.64 \pm 0.07 \mathrm{~m} / \mathrm{s} ; t=0.0501, p=0.9607$. N, TrkB.DN mice spent significantly less time in the center of the open field box during the first 3 min than eYFP mice: eYFP: $1.90 \pm 0.31 \%$; trkB.DN: $1.03 \pm 0.23 \%$ of time; $t=2.177, p=0.0458$. 0 - $\mathbf{Q}$, Elevated plus-maze. Same mice as in $\boldsymbol{A}-$ $\boldsymbol{J}$, performed d71. O, TrkB.DN mice entered the open arms significantly fewer times than eYFP mice: eYFP: $23.44 \pm 2.77$ entries; trkB.DN: $15.38 \pm 1.83$ entries; $t=2.364, p=0.0320$. $P$, TrkB. DN mice made significantly fewer head-dips toward the open arms than eYFP mice: eYFP: $36 \pm 4$ head-dips; trkB.DN: $28 \pm 3$ head-dips; $U=15.5, p=0.0490$. $\boldsymbol{Q}$, No difference was found in the time spent in the open arms between trkB.DN and eYFP mice: eYFP: $41.10 \pm 3.37 \%$; trkB.DN: $38.19 \pm 3.06 \%$ time spent in the open arms; $t=0.6334, p=0.5360$. Data shown as mean \pm SEM. For bar plots, unpaired $t$ test, two-tailed was used to assess significance if data passed the D'Agostino and Pearson normality test, if not, Wilcoxon rank-sum test was used. For $\boldsymbol{K}$, two-way ANOVA, Sidak's multiple comparisons test was used. For paired data, a Wilcoxon matched pairs test was used. Source data can be found in Extended Data Figure 5-1; ${ }^{*} p<0.05$.

synaptic contacts (Fig. $3 L, M$ ). In summary, while the postnatal deletion of trkB in PV neurons results in reduced neurite numbers (Tan et al., 2018), we show here that adult reduction of $\mathrm{BDNF} / \mathrm{trkB}$ signaling results in increased complexity of both axons and dendrites, demonstrating that this signaling pathway is not only necessary for developmental maturation but also maintenance of PV neuron integrity in the adult.

\section{Alterations in intrinsic properties and synaptic connectivity of mPFC PV interneurons}

To probe the effect of trkB.DN expression on the single neuron and local circuit level, we conducted whole-cell patch-clamp recordings of $\mathrm{mPFC} \mathrm{PV}$ interneurons and PNs. To investigate the integrity of the excitatory/inhibitory $(\mathrm{E} / \mathrm{I})$ balance in the local circuit, we used voltage-clamp experiments to record 

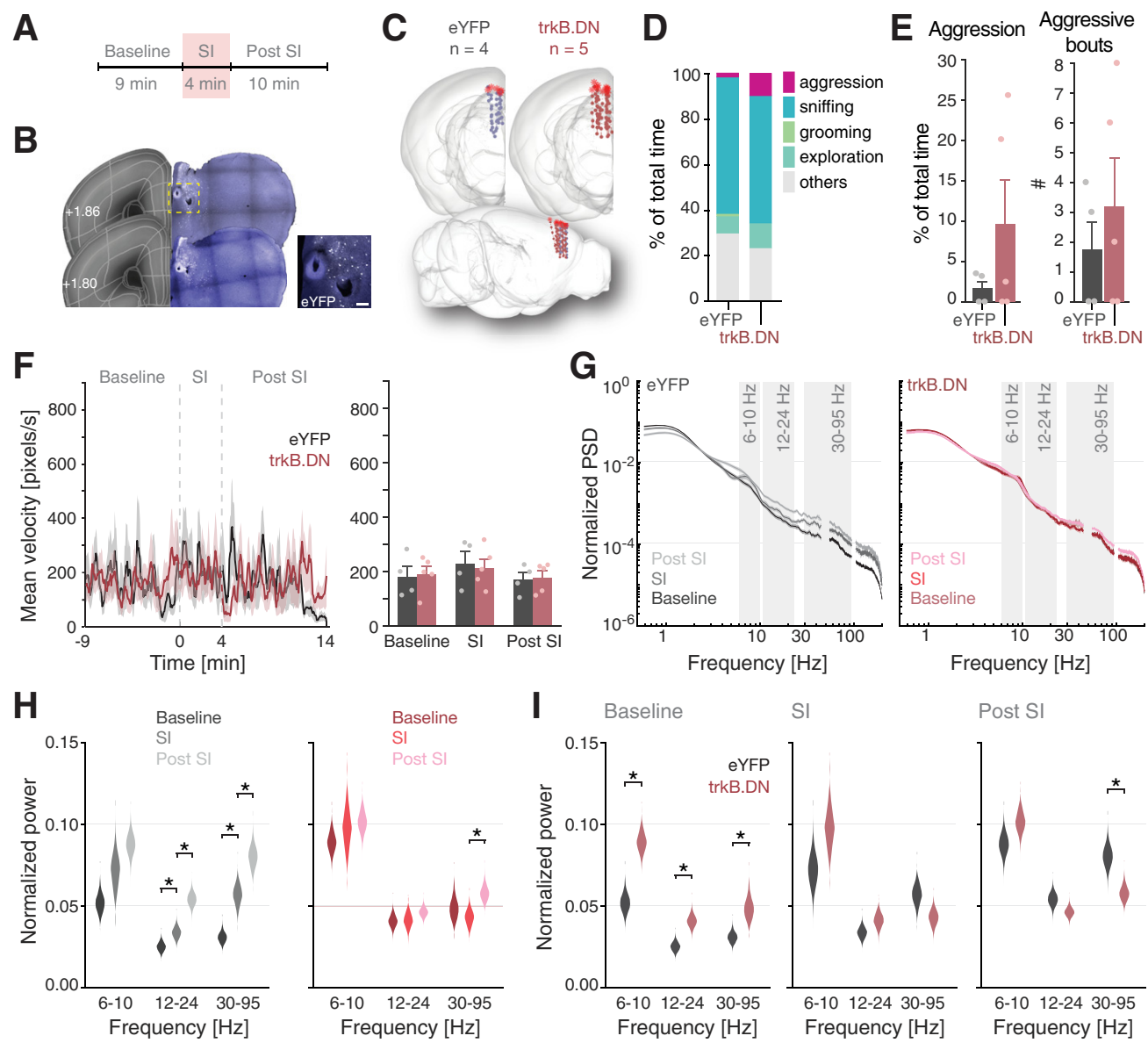

Figure 6. Alterations in LFP activities in trkB.DN mice during SI. $A-I$, Electrophysiological tetrode recording during the resident-intruder procedure. The experiment was performed once per animal, 50-60 d after viral injection. eYFP mice (gray): $n=4$ males, trkB.DN mice (red): $n=5$ males. $A$, Experimental timeline. Baseline: $-9-0$ min before introduction of the intruder; SI $0-$ $4 \mathrm{~min}$; post-SI 4-14 min after removal of the intruder. $\boldsymbol{B}$, Example brain sections from an eYFP mouse with electrolytic lesions used to reconstruct the tetrodes positions. $C$, 3D illustrations of the reconstructed tetrodes positions. Top, View from the front. Bottom, View from the right side. $\boldsymbol{D}$, Percentage of total time spent in non-social (grooming, digging, cage exploration) and social (aggression, tail rattling, sniffing) behaviors for trkB.DN and eYFP mice during SI ( $4 \mathrm{~min})$. Sniffing: eYFP: $60.3 \pm 6.4 \%$; trkB.DN: $56.1 \pm 8.2 \%$; $t=0.38, p=0.7125 ;$ grooming: eYFP: $0.8 \pm 0.5 \%$; trkB.DN: $0.0 \% ; t=1.73, p=0.1279$; exploration: eYFP: $7.6 \pm 2.1 \%$; trkB.DN: $11.0 \pm 5.6 \% ; t=-0.51, p=0.6242$; others: eYFP: $29.6 \pm 4.7 \%$; trkB.DN: $23.1 \pm 5.1 \% ; t=0.91$, $p=0.3907$. No digging or tail rattling was observed. $\boldsymbol{E}$, left, Percentage time in aggression: eYFP: $1.7 \pm 1.0 \%$; trkB.DN: $9.8 \pm 5.5 \%$ of time; $t=-1.27, p=0.2452$. Right, Number of aggressive bouts: eYFP: $1.8 \pm 0.5$; trkB.DN: $3.2 \pm 0.7$ bouts; $t=0.71, p=0.503$. $\boldsymbol{F}$, There was no difference in the mean velocity of locomotion between implanted eYFP mice and trkB.DN mice during baseline, SI, or post-SI: baseline: eYFP: $179.8 \pm 38.5$; trkB.DN: $189.8 \pm 29.1$ pixels/s; $t=0.21, p=0.839$, SI: eYFP: $229.3 \pm 42.5 ;$ trkB.DN: $212.8 \pm 32.9$ pixels/s; $t=-0.31, p=0.764$, post-SI: eYFP: $169.5 \pm 26.5$; trkB.DN: $177.3 \pm 24.1$ pixels/s; $t=0.22, p=0.834$. G-I, LFP activities during SI. G, Bootstrapped normalized PSD of the LFP for baseline, SI, and post-SI for eYFP (left) and trkB.DN mice (right). Gray shading: the $\theta(6-10 \mathrm{~Hz}), \beta(12-24 \mathrm{~Hz})$, and $\gamma(30-95 \mathrm{~Hz})$ band, respectively. Left, SI increases the power across many frequencies in eYFP mice, with further increases post-SI. Right, SI induces limited power changes in trkB.DN mice. $\boldsymbol{H}, \boldsymbol{I}$, The distribution of the PSD in specific frequency bands $(\theta, \beta$, and $\gamma$, respectively) during baseline, SI, and post-SI for eYFP (grays) and trkB.DN (reds) mice. $\boldsymbol{H}$, Comparison of the PSDs in the three frequency bands during baseline, SI, and post-SI for eYFP mice (left) and trkB.DN mice (right), respectively. SI significantly increased the power in the $\beta$ and $\gamma$ bands in the eYFP mice. The only significant change in the trkB.DN mice was an increase in $\gamma$ power after SI. $I$, Direct comparison of the PSDs in the three frequency bands between eYFP mice (gray) and trkB.DN mice (red) during baseline, SI, and post-SI. trkB.DN mice have significantly increased baseline activity in all three frequency bands compared with eYFP mice. SI increases the power in the eYFP mice, but not in trkB.DN mice, diminishing the power difference between the two groups of mice. The power increases further in eYFP mice after SI, with eYFP mice displaying significantly higher $\gamma$ power than trkB.DN mice post-SI (despite the baseline $\gamma$ power being significantly higher in trkB.DN mice than in eYFP mice). Individual data point and confidence interval values for $\theta, \beta$, and $\gamma$ range power intragroup ( $\boldsymbol{H})$ and intergroup $(\boldsymbol{I})$ comparisons can be found in Extended Data Figures 1-1, 6-1. Data shown as mean \pm SEM for $\boldsymbol{E}-\mathbf{G}$. Data shown as mean \pm SD for $\boldsymbol{H}, \boldsymbol{I}$. For bar plots, Unpaired $t$ test, two-tailed was used to assess significance if data passed the Kolmogorov-Smirnov normality test, if not, the Wilcoxon test was used. For $\boldsymbol{H}, \boldsymbol{I}$, confidence intervals of bootstrapped data were used to assess significance. Source data can be found in Extended Data Figure 6-1. Scale bar: $100 \mu \mathrm{m}(\boldsymbol{B}) ;{ }^{*}$ confidence interval not including 0.

spontaneous EPSCs and IPSCs in PNs in trkB.DN $(n=14$ in 3 mice) and eYFP ( $n=15$ in 3 mice) mice (Fig. $4 A$ ). Neurons were recorded in layer 2/3-6, with a dominance in layer 5 (Fig. 2A). We found the frequency of IPSCs to be considerably (albeit nonsignificant; $p=0.0521$ ) reduced in PNs in trkB.DN mice compared with in eYFP mice (Fig. $4 B, C$ ) while the frequency of EPSCs in PNs was similar in the two groups of mice (Fig. 4D). However, the EPSC frequencies were very low, precluding proper evaluation of any differences in spontaneous excitatory events between the two groups of mice. We next investigated the intrinsic properties of mPFC PV interneurons $(n=19$ in 6 trkB.
DN mice, $n=14$ in 10 eYFP mice; Fig. $4 E$ ). Current-clamp recordings revealed significantly reduced action potential (AP) threshold in PV interneurons in trkB.DN mice compared with in eYFP mice (Fig. 4F). Further, the PV interneurons in trkB.DN mice displayed significantly increased membrane time constant (Fig. 4G). We found the increased membrane time constant in trkB.DN mice to be a result of a significantly increased membrane capacitance rather than a change in the input resistance (Fig. 4H,I). Investigation of the properties of the rheobasic AP waveform revealed a significantly faster AP downstroke, resulting in a narrower AP half-width, in trkB. 
A

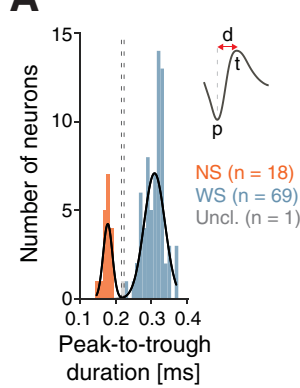

E Baseline
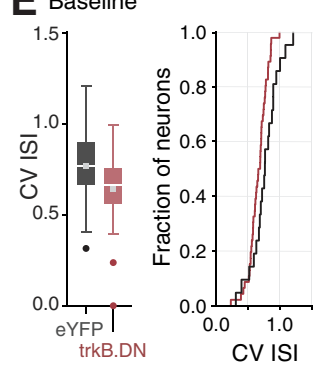

I Positively modulated

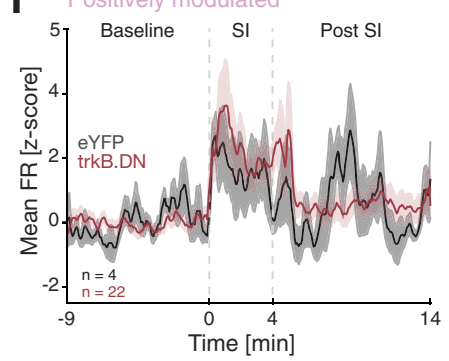

B

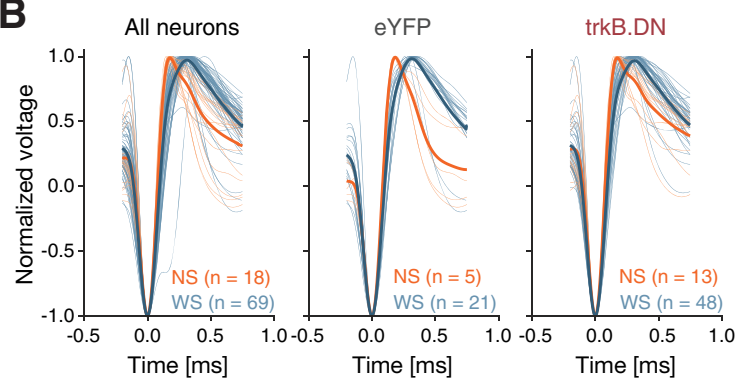

C

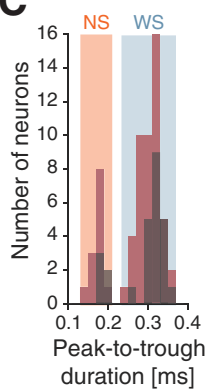

D Baseline

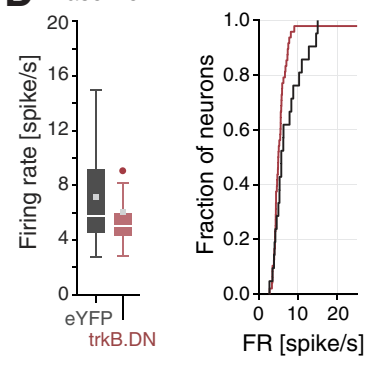

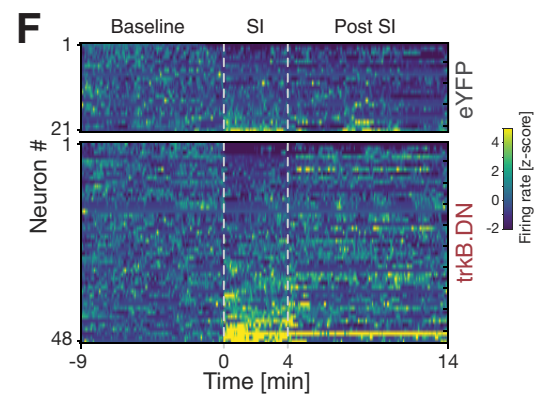

J Negatively modulated
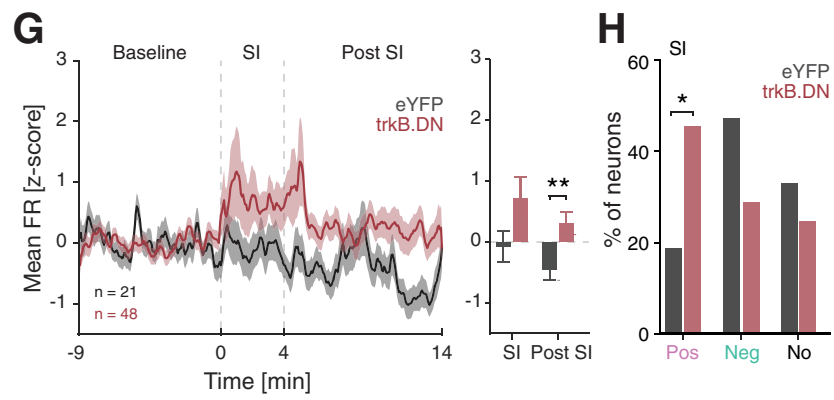

K Not modulated
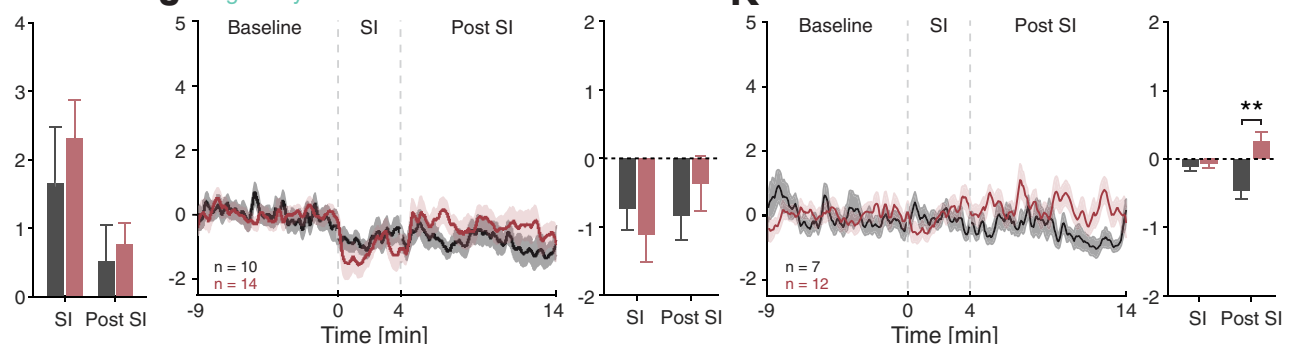

Figure 7. Alterations in single-unit and population activities in trkB.DN mice during SI. $\boldsymbol{A}-\boldsymbol{K}$, Same recordings and mice as in Figure 6. Single unit and population activities during SI. eYFP mice: gray, trkB.DN mice: red. A, Classification of recorded single-units into NS putative inhibitory interneurons and WS putative excitatory PNs based on spike waveform features [the peak to trough duration (d)]. NS (orange): $n=18$, mean $\mathrm{d}=0.177 \pm 0.012 \mathrm{~ms}$; WS (blue): $n=69$, mean $\mathrm{d}=0.309 \pm 0.028 \mathrm{~ms}$; unclassified units (gray): $n=1$. Inset, The spike waveform features used to characterize APs: peak (p), trough ( $t$ ), and peak to trough duration (d). $\boldsymbol{B}$, Normalized average spike waveforms of the classified NS (orange) and WS (blue) neurons. Left, All neurons (eYFP + trkB.DN mice), Middle, Neurons in eYFP mice. Right, Neurons in trkB.DN mice. C, The spike waveforms (peak to trough duration) of NS (orange shading) and WS (blue shading) neurons did not differ between trkB.DN (red) and eYFP (gray) mice: NS: eYFP: $0.183 \pm 0.009 \mathrm{~ms}$; trkB.DN: $0.175 \pm 0.013 \mathrm{~ms} ; t=-1.93, p=0.22 ;$ WS: eYFP: $0.319 \pm 0.025 \mathrm{~ms}$; trkB.DN: $0.305 \pm 0.029 \mathrm{~ms} ; t=-1.27 p=0.06$. $\boldsymbol{D}-\boldsymbol{K}$, Analysis of the WS neurons. $\boldsymbol{D}, \boldsymbol{E}$, There is no difference in the baseline $(-9-0 \mathrm{~min})$ firing of WS neurons between trkB.DN and eYFP mice. $\boldsymbol{D}$, left, Average firing rate of WS neurons during baseline: eYFP: $7.2 \pm 0.17$; trkB.DN: $6.08 \pm 0.12$ spike/s. Right, The cumulative distribution of the firing rate of WS neurons during baseline; KSstat $=0.32, p=0.08$. $E$, left, CV ISI distribution during baseline: eYFP: $0.76 \pm 0.01$; trkB.DN: $0.637 \pm 0.004$. Right, The cumulative distribution of the CV ISI during baseline, KS-stat $=0.31$, $p=0.1$. $A F$, The firing rate (z-scored) of individual WS neurons in eYFP mice (top: $n=21$ neurons) and trkB.DN mice (bottom: $n=48$ neurons) during baseline, SI, and post-SI. Dashed lines outline the SI period. G, Mean firing rate (z-scored) of the WS population in the eYFP and trkB.DN mice during baseline, SI, and post-SI. Left, SI significantly increased the firing rate of the WS population in trkB.DN mice but significantly decreased the firing rate of the WS population in eYFP mice. Baseline $(-9-0$ min) versus $0-14$ min $(S I+$ post-SI): eYFP: $-0.34 \pm 0.87$; $W=190, p=0.0096 ;$ trkB.DN: $0.42 \pm 0.21 ; W=368, p=0.0240$. Right, Change in mean firing rate from baseline. SI increased the WS firing in trkB.DN mice but not in eYFP mice. SI: eYFP: $-0.07 \pm 0.25$; trkB.DN: $0.71 \pm 0.35 ; U=647, p=0.06$. After SI the WS firing was significantly higher in trkB.DN mice than in eYFP mice. Post-SI: eYFP: $-0.45 \pm 0.17$; trkB.DN: $0.31 \pm 0.18 ; U=763, p=0.001$. $\boldsymbol{H}$, Based on their individual firing rate during SI (0-4 min), a significantly higher proportion of the WS neurons was positively modulated by SI in trkB.DN mice than in eYFP mice: eYFP: $19 \%$ (4 neurons); trkB.DN: $46 \%$, (22 neurons); $p=0.03, \chi^{2}=4.46$. A lower proportion of the WS neurons was negatively modulated by SI in trkB.DN mice than in eYFP mice: eYFP: $48 \%$ (10 neurons); trkB.DN: $29 \%$ (14 neurons); $p=0.14, \chi^{2}=2.19$. Not modulated neurons: eYFP: $33 \%$ (7 neurons); trkB.DN: $25 \%$ (12 neurons); $p=0.48, \chi^{2}=$ 0.51 . $\boldsymbol{I}-\boldsymbol{K}$, The firing rate of the positively, negatively, and not modulated WS subpopulations did not differ between eYFP and trkB.DN mice during SI ( $0-4 \mathrm{~min}) . \boldsymbol{I}, \mathrm{Mean}$ firing rate ( $(\mathrm{z}-\mathrm{scored})$ of positively modulated WS neuron: SI: eYFP: $1.65 \pm 0.83$; trkB.DN: $2.31 \pm 0.56$; $U=47, p=0.75$, post-SI: eYFP: $0.52 \pm 0.52$; trkB.DN: $0.76 \pm 0.32 ; U=49, p=0.86$. J, Mean firing rate (zscored) of negatively modulated WS neuron: SI: eYFP: $-0.73 \pm 0.16$; trkB.DN: $-1.12 \pm 0.32 ; U=62, p=0.66$ ), post-SI: eYFP: $-0.84 \pm 0.20 ;$ trkB.DN: $-0.37 \pm 0.30 ; U=89, p=0.28 . \boldsymbol{K}$, Mean firing rate (z-scored) of not modulated WS neuron: SI: eYFP: $-0.11 \pm 0.07$; trkB.DN: $-0.08 \pm 0.06 ; U=44, p=0.90$ ); post-SI: eYFP: $-0.46 \pm 0.12 ;$ trkB.DN: $0.26 \pm 0.14 ; U=78$, $p=0.001$. Data shown as mean \pm SEM for $(\boldsymbol{G}, \boldsymbol{I}-\boldsymbol{K}$ ). For boxplots in $\boldsymbol{D}, \boldsymbol{E}$, data shown as median (white line), mean (squared dot), box (25th and 75th percentiles), whiskers (data points that are not outliers), and dots (outliers). For cumulative distribution function in $\boldsymbol{D}, \boldsymbol{E}$, the Kolmogorov-Smirnov test was used. For bar plots in $\mathbf{G}$, Unpaired $t$ test, two-tailed was used to assess significance if data passed the Kolmogorov-Smirnov normality test, if not, the Wilcoxon test was used. For paired data in $\boldsymbol{G}$, the Wilcoxon signed-rank test was used. For $\boldsymbol{H}$, the $\chi^{2}$ proportion test was used. Source data can be found in Extended Data Figure 7-1; ${ }^{*} p<0.05,{ }^{* *} p<0.01$.

DN-expressing PV interneurons than in PV interneurons expressing eYFP (Fig. 4J,K). Surprisingly, probing of excitability on higher gain levels (by injection of larger depolarizing currents) revealed that PV interneurons expressing trkB. DN fired at significantly lower frequencies (by $\sim 80 \mathrm{~Hz}$ ) than PV interneurons expressing eYFP (Fig. $4 L, M$ ), an unexpected finding in light of the narrower AP waveform of trkB.DN- expressing PV interneurons. Furthermore, we also observed a significantly increased firing rate adaptation in the trkB.DNexpressing PV interneurons compared to eYFP expressing PV interneurons (Fig. 4N,O). Of note, one of the key characteristics of cortical PV interneurons is their fast firing rate with minor or no adaptation (Kawaguchi and Kubota, 1997). Analysis of the EPSP frequency in the PV interneurons revealed a minor 
increase in the trkB.DN mice compared with the eYFP mice (Fig. $4 P$ ).

We next used paired whole-cell recordings to investigate the effects of reduced $\mathrm{BDNF} / \mathrm{trkB}$ signaling on the synaptic connectivity between PV interneurons and target PNs $(n=10 \mathrm{PV}-\mathrm{PN}$ pairs in 8 eYFP mice, $n=8 \mathrm{PV}$-PN pairs in 7 trkB.DN mice; Fig. 4Q). To probe the functional kinetics of PV-PN synapses, APs were evoked in the PV interneurons, and IPSPs were monitored in postsynaptic PNs (Fig. 4R). No significant difference was found in the amplitude of the first elicited IPSP between trkB.DN and eYFP mice (Fig. $4 S$ ), nor in the pairedpulse ratio (Fig. 4T). This indicates that reduced $\mathrm{BDNF} / \mathrm{trkB}$ signaling in adult mPFC PV interneurons did not affect short-term synaptic plasticity of PV-PN connections. However, we detected a significantly longer IPSP decay time constant in the PNs in trkB.DN mice compared with in eYFP mice (Fig. $4 U$ ). We hypothesize that the increased IPSP decay time constant reflects adaptations in the PV-PN synapse compensating for the lower firing frequencies of $\mathrm{mPFC} P \mathrm{PV}$ interneurons expressing trkB.DN. Thus, altered PV inhibition was not readily evident at the PV-PN synapse, despite the somatic GABA levels being reduced (Fig. $1 J)$. Of relevance, GABA is synthesized (by the enzymes GAD65 and GAD67, respectively) within different (sub)cellular compartments and with different functional purposes (Lee et al., 2019), and the GABA levels in the cell body provide limited insight into GABA transmission at the synapse. Overall, the long-term effects of reduced BDNF/trkB signaling were most clearly revealed in the changed intrinsic biophysical properties of PV interneurons, changes rendering the PV interneurons less responsive to incoming activity. Several of the results indicate deficient inhibitory activity in the local network, including the trend toward a reduction in IPSC frequency in PNs, as well as the significantly reduced firing output and increased firing adaptation of the $\mathrm{PV}$ interneurons in response to increased current injection.

\section{Alterations in SI}

The results of our morphologic and ex vivo studies imply that $\mathrm{BDNF} / \mathrm{trkB}$ signaling is necessary for proper PV integrity and function in adult cortical networks, and we questioned how the observed alterations would manifest at the behavioral level. We observed that trkB.DN mice displayed increased aggression in social contexts in the homecage. Deficits in SIs and increased aggression have been linked to reduced levels of BDNF and trkB in the mPFC (Mikics et al., 2018), and to directly investigate a possible link between altered BDNF/trkB signaling in mPFC PV interneurons and changed social cognition, trkB.DN and eYFP mice were subjected to a well-validated test of SI, the resident-intruder procedure (Fig. 5A; Materials and Methods; Winslow, 2003). As a first step, the baseline aggression was scored in a iring rate

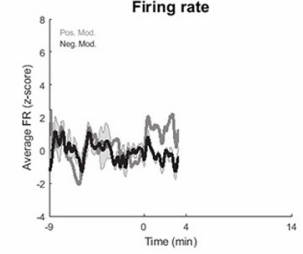

uronal Trajectory

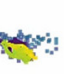

,
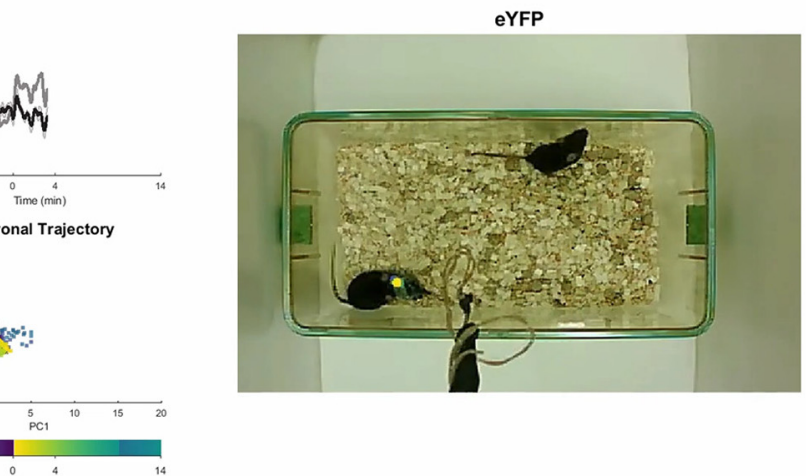

4

Firing rate

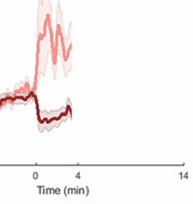

euronal Trajectory
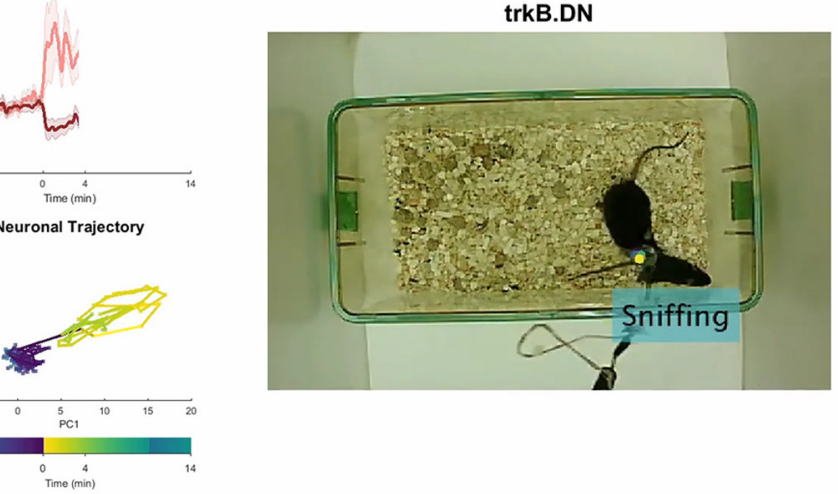

trkB.DN

Movie 1. Evolvement of firing and population dynamics trajectories of mPFC WS populations together with behavior during the resident-intruder procedure. The evolution over time of the firing (mean firing rate; z-scored) of positively and negatively modulated WS neurons, respectively, and of the population dynamics trajectories of the whole WS population, during The locomotion of the resident mouse (recorded eYFP or trkB.DN mouse; yellow tracking dot) and its interactions with the juvenile intruder were scored offline. Video is accelerated 10 times. [View online]

cohort of PV-Cre mice $(n=19)$. For this, the mice were individually exposed for $4 \mathrm{~min}$ to an unfamiliar juvenile intruder mouse (approximately four weeks old), a social stimulus that normally does not elicit aggressive responses (Winslow, 2003). Mice showing high levels of aggression were excluded $(n=2)$. The remaining mice were separated into two groups and injected with AAVDIO-eYFP ( $n=9$ mice) or AAV-DIO-trkB.DN-mCherry $(n=8$ mice) into the mPFC. All mice were thereafter subjected to the resident-intruder procedure on four additional occasions over the next $49 \mathrm{~d}$, using different juvenile intruders at every occasion (Fig. $5 A$ ). Social behaviors (sniffing, tail rattling, and aggression) and non-social behaviors (cage exploration, digging, and grooming) were scored for all five test occasions, with a focus on the longest time point after viral injections [day 49 (d49); post; Fig. 5A,B]. No significant behavioral differences were detected between trkB.DN and eYFP mice, except for measures of aggression (Fig. 5B-J). TrkB.DN mice spent more time engaged in aggressive behavior compared with eYFP mice at all tested time points (Fig. $5 C$ ), with a significant increase at $\mathrm{d} 49$ (Fig. 5D). TrkB.DN mice also conducted a higher number of aggressive bouts (Fig. $5 E$ ), with a shorter latency to the first attack, compared with eYFP mice (Fig. $5 F)$. TrkB signaling mediates effects of stress, notably in the mPFC (Barfield and Gourley, 2018). The same mice were therefore subjected to the open field test and tested in the elevated plus-maze (Fig. $5 K-Q$ ). No differences in locomotion were observed between trkB.DN and eYFP mice (Fig. $5 K$ ), and trkB.DN mice visited the 

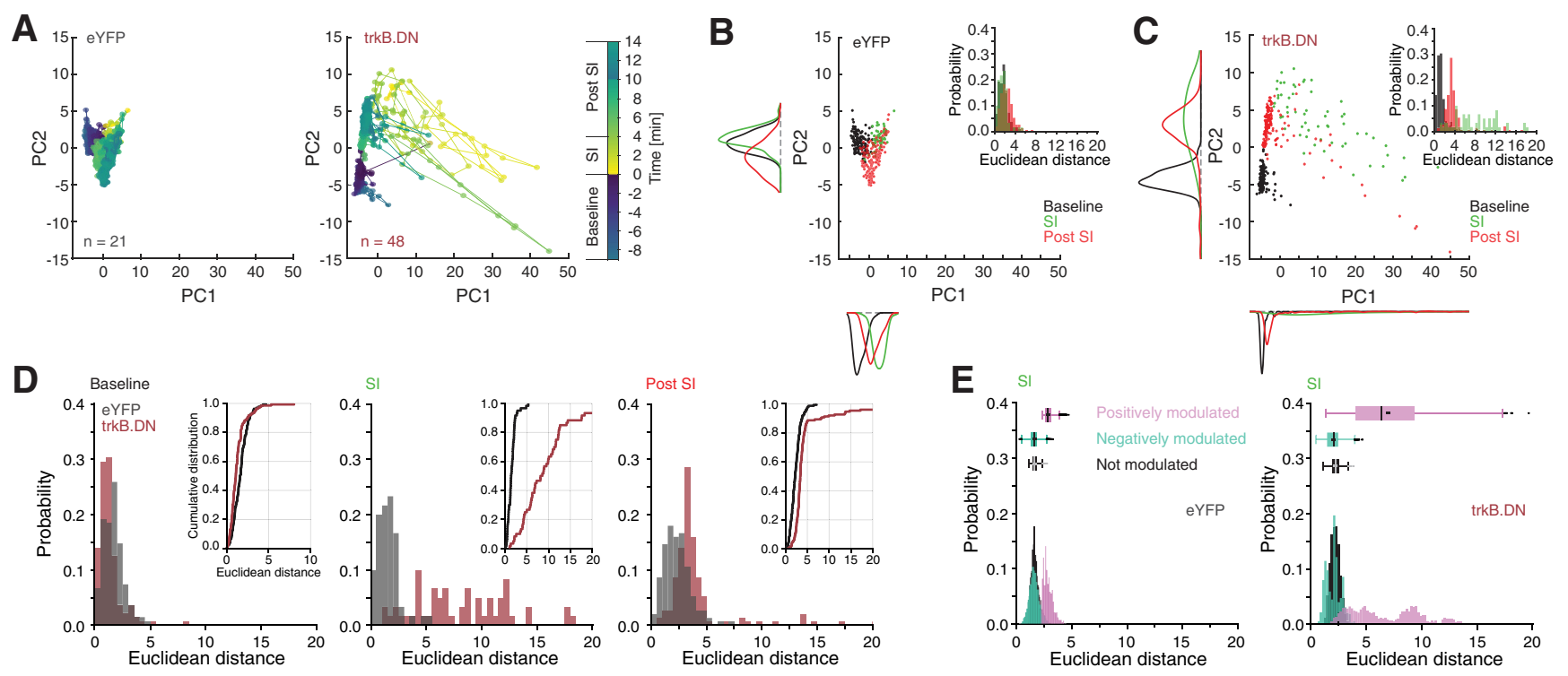

Figure 8. Alterations in WS population dynamics in trkB.DN mice during SI. A-E, Analysis of the population dynamics of the WS neurons during SI, same neurons as in Figure 7. eYFP mice: gray, trkB.DN mice: red. $A, 2 D$ projection of the neuronal population trajectories of the response patterns of WS neurons in eYFP mice (left) and trkB.DN mice (right) during the resident-intruder procedure, with color coding of the timeline (bin size: 4 s). PC1 and PC2 explained, respectively, $23.4 \%$ and $14.0 \%$ of variance seen in the eYFP mice, while PC1 and PC2 explained, respectively, $42.8 \%$ and $11.3 \%$ of variance in the trkB.DN mice. $B, C$, Scatter plot and kernel density estimation of the 2 D projection of the population dynamics (bin size: 4 s). Each data point corresponds to the first and second PC, color coded according to the behavioral epoch (baseline, black; SI, green; and post-SI, red). $\boldsymbol{B}$, In eYFP mice, the population activity patterns of the three behavioral epochs separated into defined but overlapping clusters. Inset, The probability distribution of the Euclidean distance differed between the behavioral epochs (one-way ANOVA, $F_{\text {cluster }(2,342)}=$ $19.1, p<0.001)$, with a significant difference between $S I$ and post-SI $(p<0.001)$, and baseline and post-SI $(p<0.001)$. However, the probability distribution of the Euclidean distance of the baseline and SI did not differ $(p=1)$. C, In trkB.DN mice, the population activities during baseline clustered together, with minimal overlap with the activity patterns during SI and post-SI. The population activities during SI and post-SI were considerably more variable but did yet separate. Inlet, The probability distribution of the Euclidean distance significantly differed between the three behavioral epoch (one-way ANOVA, $F_{\text {cluster(2,342) }}=52.7, p<0.001$ ), with significant differences between baseline and $\mathrm{SI}(p<0.001)$, SI and post-SI $(p<0.001)$, and baseline and post-SI $(p<0.001)$. D, Comparison of the probability distribution of the Euclidean distance for each behavioral epoch [baseline (left), SI (middle), and post-SI (right)] revealed significant differences between eYFP and trkB.DN mice, particularly during SI [inset, cumulative distribution function (CDF) comparison: $\mathrm{KS}$-stat $=0.85, p=3.15 \times 10^{-20}$ ]. Baseline (inset, $\mathrm{CDF}$ comparison: KSstat $=0.31, p=2.7 \times 10^{-6}$ ); post-SI (inset, CDF comparison: KS-stat $=0.47, p=1.8 \times 10^{-15}$ ). $\boldsymbol{E}$, To separate the population dynamics of the positively, negatively, and not modulated WS subpopulations during $\mathrm{SI}$, a bootstrapped version of the trajectory analysis was performed. This revealed high variability in the Euclidean distance of specifically the positively modulated WS neurons in the trkB.DN mice (right), variability not present in the eYFP mice (left). For $\boldsymbol{B}, \boldsymbol{C}$, one-way ANOVA was used along with a Bonferroni post hoc test. For CDF plots (D), the Kolmogorov-Smirnov test was used. Source data can be found in Extended Data Figure 8-1.

center of the open field in the same manner as the eYFP mice within the first $3 \mathrm{~min}$ of the test (Fig. $5 L, M$ ). However, trkB.DN mice spent significantly less time in the center (Fig. $5 N$ ), possibly indicating changes in anxiety. In support of increased anxiety, trkB.DN mice visited the open arms in the elevated plus-maze significantly fewer times than eYFP mice, and also performed fewer head-dips over the edges of the open areas, a sign of increased anxiety (Rodgers and Dalvi, 1997; Fig. 5O,P). However, the total time spent in the open arms was not significantly changed (Fig. $5 Q$ ). In summary, our findings directly link BDNF/trkB signaling in $\mathrm{mPFC} \mathrm{PV}$ interneurons to aggression and also point to a possible role in anxiety-related behavior.

\section{Alterations in prefrontal network responses during SI}

To directly study mPFC responses and network dynamics during SI, we recorded LFP and single-unit activity before ( $9 \mathrm{~min}$; baseline), during ( $4 \mathrm{~min}$; SI), and after SI (10 min; post-SI; Fig. $6 \mathrm{~A}-\mathrm{C}$ ) using the resident-intruder procedure, in new cohorts of mice (trkB.DN; $n=5$, eYFP; $n=4$ mice). As in the earlier experiments (Fig. 5), the mice exhibited social and non-social behaviors, confirming that the tetrode implant did not overly affect the behavior (Fig. 6D). Further, akin to our earlier results (Fig. 5C-F), trkB.DN mice exhibited increased aggression with a higher number of aggressive bouts compared with eYFP mice (Fig. 6E). No differences in locomotion between trkB.DN and eYFP mice were detected (Fig. 6F). Analysis of the LFP oscillatory patterns revealed that SI evoked increased power over a broad frequency spectrum in eYFP mice, with a significant increase specifically in the $\beta(12-24 \mathrm{~Hz})$ and $\gamma(30-95 \mathrm{~Hz})$ bands, an increase that became even more prominent after the intruder was removed (post-SI; Fig. 6G,H). In contrast, SI did, at large, not change the LFP power in trkB.DN mice. The only significant power change detected was an increase in $\gamma$ activity after SI (post-SI; Fig. $6 H$ ). Importantly, a direct comparison of LFP oscillations between trkB.DN and eYFP mice revealed significantly increased baseline power over a broad frequency spectrum in trkB.DN mice (Fig. $6 I$ ). Compared with the eYFP mice, the trkB.DN mice, thus, presented enhanced baseline activity and deficient induction of synchronous activities over a broad frequency band in response to social stimuli (Fig. 6I).

We next analyzed single-unit activities during SI. The recorded $\mathrm{mPFC}$ units were pooled and classified into NS putative inhibitory interneurons $(n=18)$ and WS putative PNs $(n=69)$ based on spike waveform features (Ardid et al., 2015; Materials and Methods) for both trkB.DN ( $n=48 \mathrm{WS}, 13 \mathrm{NS}$ ) and eYFP mice ( $n=21$ WS, 5 NS; Fig. 7A-C). Because of their low number, the NS units were excluded from further analyses. No significant differences were detected in the baseline activity (mean firing rate, and the CV of the ISI) of the mPFC WS neurons between trkB.DN and eYFP mice (Fig. $7 D, E$ ). However, SI increased the mean firing rate of the WS population in the trkB.DN mice, but not in the eYFP mice (Fig. $7 F, G$ ). Investigation of the individual WS neurons revealed that a significantly higher proportion of the WS neurons were positively modulated by SI in the trkB.DN 

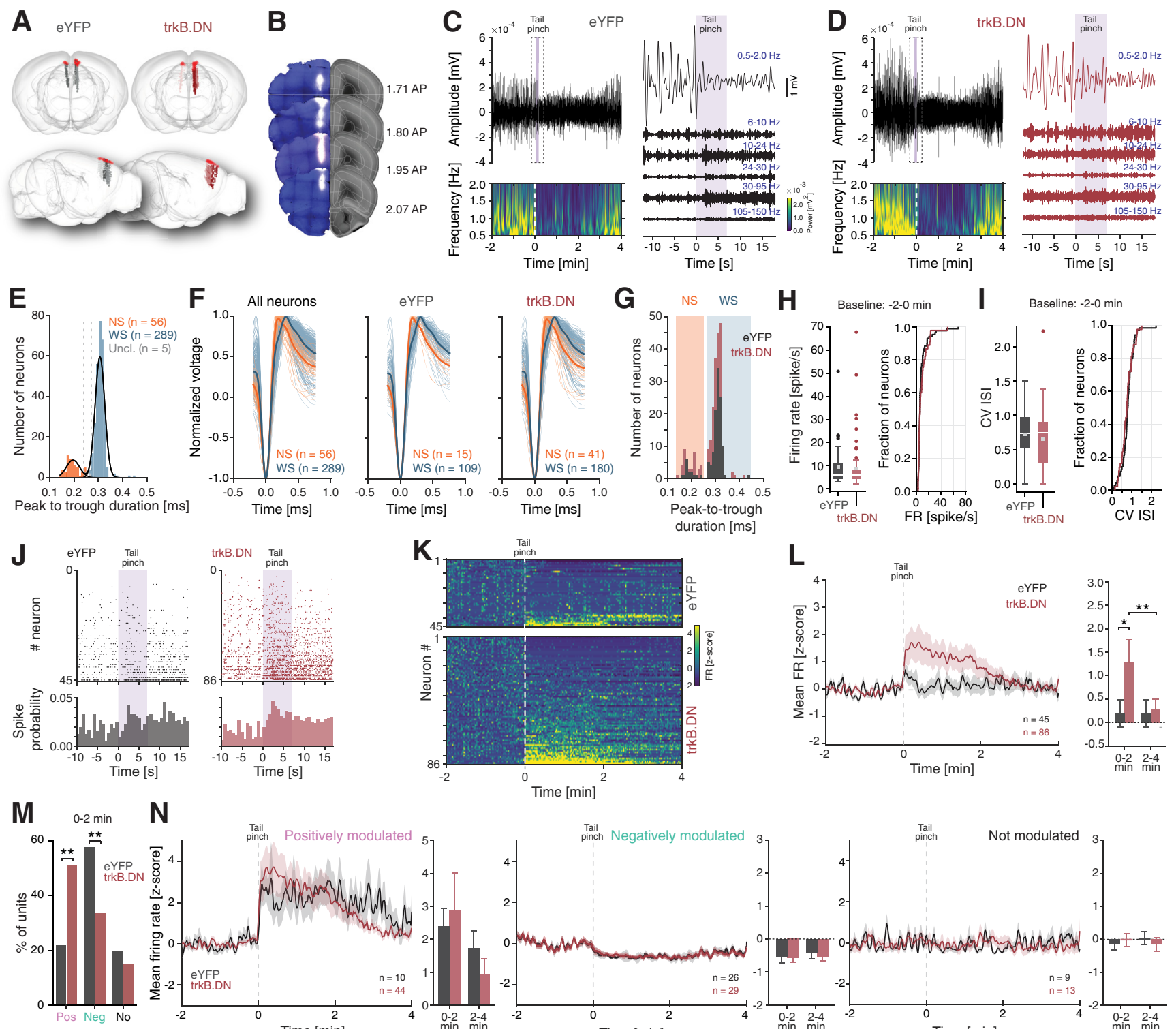

$\mathbf{N}$
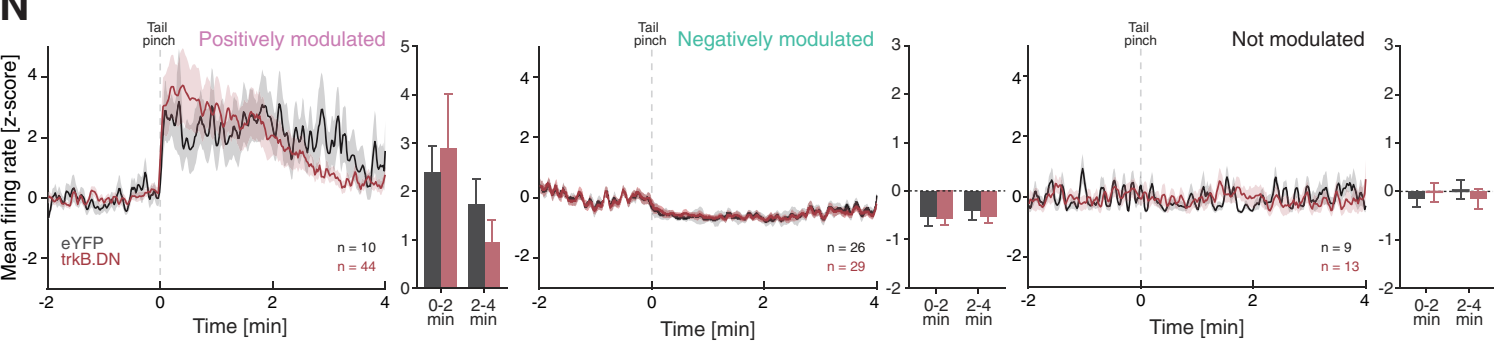

Figure 9. Alterations in single-unit activities in trkB.DN mice in response to tail pinch. $\boldsymbol{A}-\boldsymbol{N}$, Extracellular single-unit and LFP recordings under urethane anesthesia. eYFP mice (gray): $n=4$ males, trkB.DN mice (red): $n=5$ males. $A$, 3D illustrations of the reconstructed probe positions. Top, View from the front. Bottom, View from the right side. $\boldsymbol{B}$, Example brain sections from an eYFP mouse with Dil labeling (white) of the tracts from the four-shank silicon probe. $\boldsymbol{C}, \boldsymbol{D}$, Representative LFP recording (6 min shown) from an eYFP (C) and a trkB.DN (D) mouse before, during, and after tail pinch. Violet vertical bar, tail pinch (7 s). Left, top, Filtered (0-200 Hz) LFP. Dashed box, Shown in closeup on the right. Bottom, Wavelet spectrogram of LFP showing the decrease in power in slow frequencies $(0.5-2.0 \mathrm{~Hz})$ in response to tail pinch. Dashed line: start of tail pinch. Right, Close up of the dashed box in top left. Filtering of the LFP into different frequency bands. $E$, Classification of recorded single-units into NS, putative inhibitory interneurons and WS putative excitatory PNs based on spike waveform features [the peak to trough duration (d)]. NS (orange): $n=56$, mean $\mathrm{d}=0.194 \pm 0.025 \mathrm{~ms}$; WS (blue): $n=289$, mean $\mathrm{d}=0.307 \pm 0.019 \mathrm{~ms}$; gray: unclassified units $(n=5)$. $\boldsymbol{F}$, Normalized average spike waveforms of the classified NS (orange) and WS (blue) neurons. Left, All neurons (eYFP + trkB.DN mice). Middle, Neurons in eYFP mice. Right, Neurons in trkB.DN mice. G, The spike waveforms (peak to trough duration) of NS (orange shading) and WS (blue shading) neurons do not differ between trkB.DN (red) and eYFP mice (gray): NS: eYFP: $0.191 \pm 0.021 \mathrm{~ms} ;$ trkB.DN: $0.195 \pm 0.027 \mathrm{~ms}$; $U=8983$, $p=0.23$, WS: eYFP: $0.309 \pm 0.019 \mathrm{~ms}$; trkB.DN: $0.306 \pm 0.019 \mathrm{~ms} ; \boldsymbol{U}=1063, p=0.80$. $\boldsymbol{H}-\boldsymbol{N}$, Activity of WS neurons. $\boldsymbol{H}, \boldsymbol{I}$, There is no difference in the baseline ( $-2-0$ min) firing of WS neurons between trkB.DN (red) and eYFP (gray) mice. $\boldsymbol{H}$, left, Average firing rate of WS neurons during baseline: eYFP: $9.28 \pm 0.19$; trkB.DN: $8.67 \pm 0.11$ spike/s. Right, The cumulative distribution of the firing rate of WS neurons during baseline; KS-stat $=0.16, p=0.43$. I, left, CV ISI distribution during baseline: eYFP: $0.72 \pm 0.01$; trkB.DN: $0.65 \pm 0.01$. Right, The cumulative distribution of the CV ISI during baseline, KS-stat $=0.15, p=0.58$. J, Top, Raster plot of the spiking of the WS neurons in eYFP mice (gray) and trkB.DN mice (red) before, during (violet vertical bar, $7 \mathrm{~s}$ ), and after tail pinch. Bottom, The spike probability. $\boldsymbol{K}$, The firing rate (z-scored) of individual WS neurons in eYFP mice (top: $n=45$ neurons) and trkB.DN mice (bottom: $n=86$ neurons) before, during, and after tail pinch. Dashed line: start of tail pinch. $L$, Mean firing rate (z-scored) of the WS population in eYFP and trkB.DN mice. Left, Tail pinch increased the firing rate of the WS population to a higher degree in trkB.DN mice than in eYFP mice. Dashed line: start of tail pinch. Right, The WS population in trkB.DN mice had a significantly higher firing rate than the WS population in eYFP mice $0-2$ min after a tail pinch (eYFP: $0.19 \pm 0.23$; trkB.DN: $1.28 \pm 0.46 ; U=2434, p=0.01$ ), but not $2-4$ min after the tail pinch (eYFP: $0.16 \pm 0.20$; trkB.DN: $0.28 \pm 0.15 ; U=2072, p=0.51)$. The firing rate of the WS neurons in the trkB.DN mice decreased significantly after 2 min $\left(W=2567, p=6 \times 10^{-5}\right)$ but not in the eYFP mice $(W=470$, $p=0.5918) . M$, Based on the firing rate of the individual WS neurons after tail pinch $(0-2 \mathrm{~min})$, a significantly higher proportion of the WS neurons was positively modulated by SI in trkB.DN mice than in eYFP mice. eYFP: 22\% (10 neurons); trkB.DN: $51 \%$ (44 neurons), $p=0.001, \chi^{2}=10.21$. A significantly lower proportion of the WS neurons was negatively modulated by SI in trkB.DN mice than in eYFP mice. eYFP: $58 \%$, (26 neurons); trkB.DN: $34 \%$ (29 neurons), $p=0.008, \chi^{2}=7.01$. Not modulated neurons: eYFP: $20 \%$ ( 9 neurons); trkB.DN: $15 \%$ (13 neurons), $p=0.48, \chi^{2}=0.50$. $\boldsymbol{N}$, The mean firing rate (z-scored) of the positively (left), negatively (middle), and not modulated (right), WS subpopulations. No differences in the mean firing rate of the WS populations were found for any of the three subpopulations between trkB.DN and eYFP mice. Positively modulated: $0-2$ min: eYFP: $2.38 \pm 0.38 ;$ trkB.DN: $2.89 \pm 1.02 ; U=179, p=0.37$; and $2-4$ min: eYFP: $1.72 \pm 0.36$; trkB.DN: $0.95 \pm 0.30 ; U=164, p=0.22$. Negatively modulated: $0-2$ min: eYFP: $-0.13 \pm 0.10$; trkB.DN: $-0.58 \pm 0.05 ; U=292, p=0.15 ;$ and $2-4$ min: 

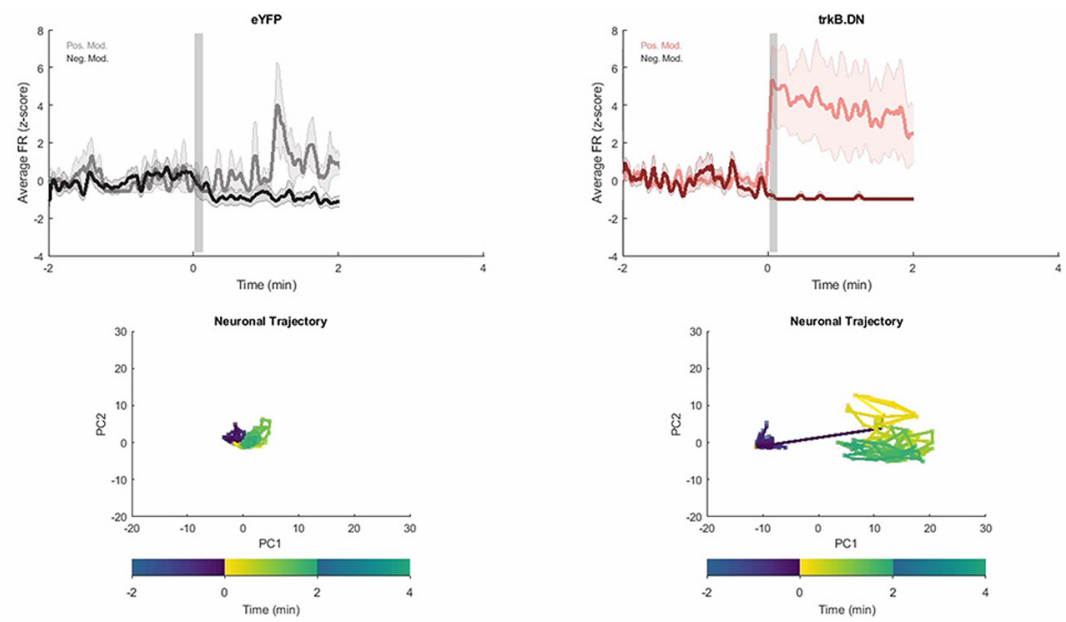

Movie 2. Evolvement of firing and population dynamics trajectories of mPFC WS populations during tail pinch. The evolution over time of the firing (mean firing rate; $z$-scored) of positively and negatively modulated WS neurons, respectively, and the population dynamics trajectories of the whole WS population, during tail pinch under urethane anesthesia (Figs. 9, 10). A representative eYFP (left), and trkB.DN mouse (right), respectively, is shown. Gray bar: tail pinch (7 s). Video is accelerated 10 times. [View online]

mice compared with in the eYFP mice (Fig. $7 H$ ). In addition, a lower proportion of WS neurons were negatively modulated by SI in the trkB.DN mice than in the eYFP mice (Fig. $7 H$ ). Importantly, the mean firing rates of the respective WS subpopulations (positively, negatively, and not modulated, respectively) did not differ between trkB.DN and eYFP mice (Fig. $7 \mathrm{H}-\mathrm{K}$; Movie 1). It is thus evident that the higher mean firing of the WS population in trkB.DN mice compared with in the eYFP mice (Fig. $7 F, G$ ) was not a result of an increased number of spikes per neuron but rather a result of a larger population of WS neurons being recruited (positively modulated) in trkB.DN mice than in eYFP mice during SI.

To further investigate how SI is represented in the MPFC WS population and to reveal potentially altered population-level dynamics in trkB.DN mice, we characterized the activity dynamics of simultaneously recorded WS mPFC neurons using neural trajectory analysis (Hamm et al., 2017; Levy et al., 2019; Pessoa, 2019). After projecting the firing rate of the WS population along time (4-s bins) onto the first two principal components (Fig. $8 A$ ), we compared the intra-cluster variability of the three clusters representing the behavioral epochs (baseline, SI, and postSI), by calculation of the distribution of the Euclidean distance from the cluster centroid (Fig. 8B,C; Materials and Methods). The variability in the distribution of the activity patterns of the WS population was significantly higher in trkB.DN mice than in eYFP mice in all three behavioral epochs, with a remarkably pronounced difference in the WS activities during SI (Fig. 8C, $D$; Movie 1). To ensure that the observed difference in the distribution of the Euclidean distance between trkB.DN and eYFP

$\leftarrow$

eYFP: $-0.40 \pm 0.14 ;$ trkB.DN: $-0.53 \pm 0.06 ; U=264, p=0.06$. Not modulated: $0-2$ min: eYFP: $-0.15 \pm 0.02$; trkB.DN: $-0.02 \pm 0.03 ; \quad U=85, p=0.08$; and $2-4 \mathrm{~min}$ : eYFP: $0.03 \pm 0.10$; trkB.DN: $-0.15 \pm 0.10 ; U=54, p=0.79$. Dashed line: start of tail pinch. Data shown as mean \pm SEM. For bar plots, the Wilcoxon rank-sum test was used to assess significance since data did not pass the Kolmogorov-Smirnov test for normality. For boxplots $(\boldsymbol{H}$, I), data shown as median (white line), mean (squared dot), box (25th and 75th percentiles), whiskers (data points that are not outliers), and dots (outliers). For paired data in ( $\boldsymbol{L}$ ), the Wilcoxon signed-rank test was used. Source data can be found in Extended Data Figure 9-1; ${ }^{*} p<0.05,{ }^{* *} p<0.01$. mice was not a mere result of the differences in the number of positively modulated WS neurons, we performed a bootstrapped version of the PCA (see Materials and Methods) to separate the dynamics of the negatively, positively and not modulated WS subpopulations during SI. This revealed that the largest dynamics divergence between the trkB. $\mathrm{DN}$ and eYFP mice was found in the positively modulated WS subpopulation, which displayed marked variability in the average Euclidean distance in trkB. $\mathrm{DN}$ mice but not in the eYFP mice (Fig. $8 E)$. Thus, neither the difference in the number of positively modulated WS neurons between trkB.DN and eYFP mice, nor the differences in the spike rate of individual neurons, were major contributors to the divergent dynamics of the WS population between trkB.DN and eYFP mice during SI (Fig. $8 A-D$ ). Instead, the altered dynamics of the WS population in trkB.DN mice were driven by increased variability in the distribution of the activity patterns specifically in the positively modulated WS subpopulation (Fig. 8E).

\section{Generalized alterations in prefrontal network responses}

We questioned whether the altered population-level dynamics observed in trkB.DN mice during SI were generalized and would also manifest during other functional states engaging separate mPFC circuitry. Tail pinch is known to induce a state of arousal, characterized by desynchronized LFP activity, reduced power of slow oscillations $(0.5-2.0 \mathrm{~Hz})$, and increased single-unit activity in the mPFC (Mantz et al., 1988; Massi et al., 2012). We recorded mPFC single-unit activity and LFP oscillations during a single tail pinch (7 s) in urethane anesthetized mice (eYFP: $n=3$; trkB. DN: $n=5$; Fig. $9 A, B)$. As expected, tail pinch reduced the power of low-frequency oscillations $(0.5-2.0 \mathrm{~Hz})$, and we also observed an increase in higher frequencies in both trkB.DN and eYFP mice (Fig. 9C,D). As before, the recorded single-units were classified into NS or WS neurons (Fig. 9E-G), and the activity of units with $>0.1$ spikes/s during baseline $(-2-0 \mathrm{~min}$ before tail pinch) was analyzed further (eYFP: $n=45 \mathrm{WS}$; trkB.DN: $n=86 \mathrm{WS}$ ), again excluding analysis of the NS, putative PV, units because of their low numbers. No significant differences were detected in the baseline activity (mean firing rate and the CV ISI) of the mPFC WS neurons between trkB.DN and eYFP mice (Fig. 9H,I). The tail pinch evoked increased firing, particularly in the trkB. DN mice (Fig. 9J-L; Movie 2), resulting in a significantly increased mean firing rate of the WS population in trkB.DN mice during the first 2-min posttail pinch compared with baseline. Paralleling the results derived by analysis of mPFC dynamics during SI, a significantly higher proportion of the WS neurons were positively modulated by tail pinch in the trkB.DN mice than in the eYFP mice (Fig. 9M). Further, a significantly lower proportion of the WS neurons in trkB.DN mice was negatively modulated by the tail pinch. Again, as seen during SI, the mean firing rate of the subpopulations of WS neurons (positively, negatively, and not modulated, respectively) did not differ between the two animal groups (Fig. 9N). We next performed trajectory analysis of the WS population activity, as before (Fig. 
A
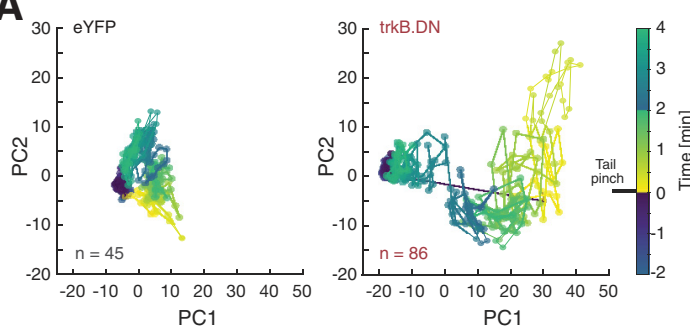

B

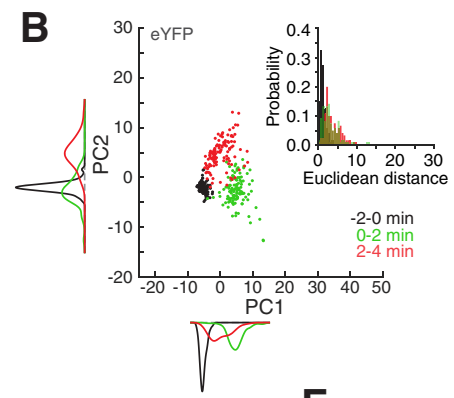

E

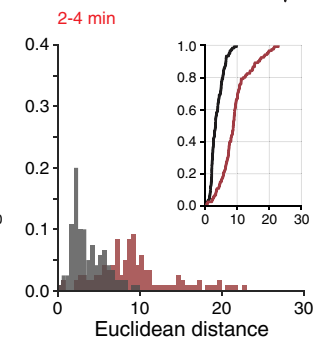

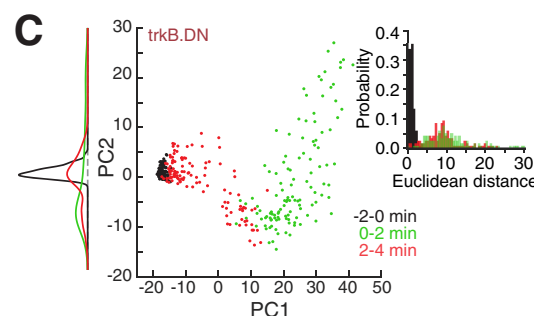

PC1
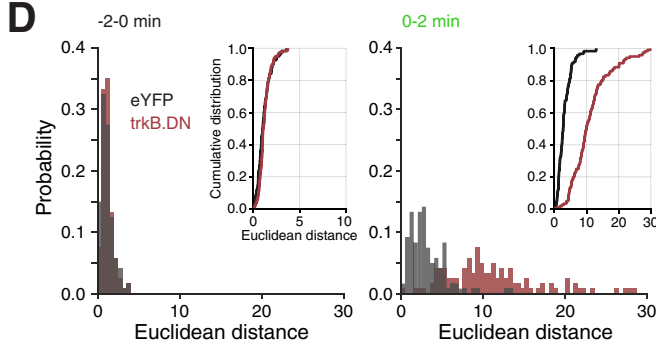

Figure 10. Alterations in WS population dynamics in trkB.DN mice in response to tail pinch. $A-E$, Analysis of the population dynamics of the WS neurons in response to tail pinch, same neurons as in Figure 9. eYFP mice: gray, trkB.DN mice: red. $A, 2 D$ projection of the neuronal population trajectories of the response patterns of WS neurons in eYFP mice (left) and trkB.DN mice (right) during the tail pinch experiment, with color coding of the timeline (bin size: 1 s). PC1 and PC2 explained, respectively, 25.2\% and 19.2\% of variance in the eYFP mice, while PC1 and PC2 explained, respectively, $68.4 \%$ and $8.6 \%$ of variance in the trkB.DN mice. $B, C$, Scatter plot and kernel density estimation of the $2 D$ projection of WS population dynamics. Each data point corresponds to the first and second $\mathrm{PC}$, color coded according to the temporal intervals ( $-2-0 \mathrm{~min}$, black; $0-2 \mathrm{~min}$, green; and 2-4 min, red). $\boldsymbol{B}$, In eYFP mice, the population activity patterns of the three temporal intervals separated into defined clusters with little variability or overlap. Inset, The probability distribution of the Euclidean distance differed between the temporal intervals (one-way ANOVA, $F_{\text {cluster( } 2,357)}=69.4, p<0.001$ ), with significant differences between $-2-0$ and $0-2 \min (p<0.001)$, between $-2-0$ and $2-4$ min $(p<0.001$ ), and between $0-2$ and $2-4 \mathrm{~min}(p=0.04)$. C, In trkB.DN mice, the population activities during baseline clustered together, with minimal overlap with the activity patterns during SI and post-SI. The population activities during $\mathrm{SI}$ and post-SI were considerably more variable but did yet separate. Inset, The probability distribution of Euclidean distance significantly differed between the temporal intervals (one-way ANOVA, $F_{\text {cluster( } 2,357)}=167.8, p<0.001$ ), with significant differences between $-2-0$ and $0-2 \min (p<0.001)$, between $0-2$ and $2-4$ min $(p=0.004)$, and between $-2-0$ and $2-4 \mathrm{~min}(p<0.001)$. $\boldsymbol{D}$, Comparison of the probability distribution of the Euclidean distance for each temporal interval $[-2-0 \mathrm{~min}$ (left), $0-2$ min (middle), and 2-4 min (right)] revealed significant differences between eYFP and trkB mice $0-2$ min after tail pinch [inset, cumulative distribution function (CDF) comparison: KS-stat $=0.73, p=1.97 \times 10^{-19}$ ] and $2-4$ min after tail pinch (inset, CDF comparison: KS-stat $=0.67, p=2.13 \times 10^{-24}$ ). $-2-0$ min (inset, $(D F$ comparison: KS-stat $=0.16, p=0.089$ ). $\boldsymbol{E}$, To separate the population dynamics of the positively, negatively, and not modulated WS subpopulations during $0-2 \mathrm{~min}$ after tail pinch, a bootstrapped version of the trajectory analysis was performed. This revealed high variability in the Euclidean distance of specifically the positively modulated WS neurons in the trkB.DN mice (right), variability not present in the eYFP mice (left). For $\boldsymbol{B}$, $\boldsymbol{C}$, one-way ANOVA was used along with a Bonferroni post hoc test. For the CDF plots (D), Kolmogorov-Smirnov test was used to assess significance. Source data can be found in Extended Data Figure 10-1.

$8 A-D$ ). The firing rate of the WS population was projected along time (1-s bins) onto the first two principal components (Fig. $10 A$ ), and the intracluster variability of the clusters representing three temporal intervals [ $-2-0 \mathrm{~min}$ (baseline), $0-2 \mathrm{~min}$, and 2$4 \mathrm{~min}$; start of tail pinch $=0 \mathrm{~min}]$ was compared (Fig. 10B,C). Our analyses revealed abnormalities in the population-level dynamics of the mPFC WS neurons in trkB.DN mice similar to was what seen during SI, with the tail pinch (0-2 and 2-4 min) significantly increasing the variability in the distribution of the activity patterns in trkB.DN mice compared with in eYFP mice (Fig. 10D; Movie 2). The bootstrapped version of the PCA revealed, again, that the largest divergence between the trkB.DN and eYFP mice was found in the dynamics of the positively modulated WS neurons, which displayed dynamics and variability in the distribution of the average Euclidean distance in trkB. DN mice that were not seen in the eYFP mice (Fig. 10E).

\section{Discussion}

The present study provides evidence for an essential role of $\mathrm{BDNF} /$ trkB signaling in the maintenance and function of prefrontal PV interneurons in the adult brain. The functional alterations of $\mathrm{PV}$ interneurons in response to reduced $\mathrm{BDNF} / \mathrm{trkB}$ signaling were particularly manifested under conditions with increased drive of neuronal activity, in our study exemplified by SI or tail pinch. Interestingly, the functional alterations resulted in deviations in the network dynamics that appeared to manifest across sensory stimuli and brain states. Our experimental approach excludes network alterations induced by changed differentiation or maturation of PV interneurons, or altered developmental processes depending on proper PV maturation, such as the postnatal critical period. Moreover, the approach avoids brain-wide manipulations of BDNF/trkB signaling, allowing pin-pointing of the origin of any alterations specifically to mPFC PV interneurons.

\section{BDNF/trkB signaling and mPFC network activities}

SI significantly increased the mean firing rate of the WS population in trkB.DN mice, but not in eYFP mice, and the WS firing remained elevated for minutes. Importantly, this difference in the firing activity was a result of a significantly larger proportion of WS neurons being positively modulated by SI in trkB.DN mice than in eYFP mice. Further, neural trajectory analysis revealed divergent population dynamics between trkB.DN and eYFP mice. The trajectories during and after SI separated from the baseline trajectories in trkB.DN mice, but not in eYFP mice. There was, thus, higher variability in the WS population response to SI in trkB.DN mice than in eYFP mice, and this variability was specifically found in the positively modulated WS subpopulation in trkB.DN mice. Notably, very similar alterations in WS activity and dynamics in trkB.DN mice were seen in response to a tail pinch under anesthesia. While our experiments cannot reveal whether individual neurons display similar responses to SI and tail pinch (as they were performed in different animal cohorts), the results indicate generalized alternations 
in the $\mathrm{MPFC}$ WS responses in disparate stimulus-driven brain states (social stimuli vs pure sensory stimuli, tail pinch), suggesting a general deficit in externally driven information processing (Hamm et al., 2017). Of note, all electrophysiological recordings and offline spike sorting were performed blindly with regard to the animal identity (trkB.DN vs eYFP), and disclosure of the identities revealed that a higher number of neurons were consistently recorded in the trkB.DN mice than in the eYFP mice, possibly because of the increased activity evoked in trkB.DN mice allowing more neurons to be active enough to be recorded.

Analysis of multineuronal activity patterns across time has revealed increased variability in stimulus responses in the visual cortex in mouse models of schizophrenia displaying altered inhibitory activity (Hamm et al., 2017). Interestingly, population states evoked by visual stimuli were unreliably activated in chronic mouse models of schizophrenia, but not in acute models, indicating that chronic models entail long-lasting structural changes in cortical networks that give rise to lasting generalized disorganization of functional local neuronal ensembles (Hamm et al., 2017). The structural alterations we observe after longterm (several weeks) reduction of $\mathrm{BDNF} / \mathrm{trkB}$ signaling in $\mathrm{PV}$ interneurons support this notion. The role of PV interneurons in the regulation of cortical neuronal ensembles has been directly investigated. First, optogenetic suppression of PV inhibition in the visual cortex during visual stimulation, but not during baseline, increased neuronal activity (Agetsuma et al., 2018). Similarly, we found no difference in the WS firing rate during baseline, while both SI and tail pinch significantly increased the firing rate in trkB.DN but not eYFP mice. Further, in Agetsuma and colleagues, PV suppression diminished the differential spatiotemporal population activity patterns typically seen in response to different visual stimuli, i.e., the response reliability decreased, which reduced the functional repertoire of ensembles (Agetsuma et al., 2018). Reduced stimuli differentiation has also been demonstrated in the mPFC in a mouse model of social dysfunction associated with autism (Cntnap2 knock-out; Levy et al., 2019). As in our study, the activity dynamics of simultaneously recorded $\mathrm{mPFC}$ neurons were characterized using neural trajectory analysis. Social and non-social stimuli (represented by olfactory cues) were found to evoke clearly divergent population responses in wild-type mice, a separation persisting seconds after stimulus offset. However, this category separation was decreased in the autism model, with $\mathrm{MPFC}$ neurons showing reduced differentiation between social and non-social stimuli (Levy et al., 2019). While we specifically assessed response variability, and not stimuli or category separation, the findings in the studies converge on disrupted population dynamics and disorganization of local population activity during stimuli processing. In both our study and in the study by Agetsuma et al. (2018), the altered dynamics were directly linked to changes in PV interneurons. Of interest, Agetsuma et al. (2018) suggest that as the mechanisms underlying the alterations rely on anatomic network structure, the network aberrations are independent of the cognitive or behavioral state, and should present both in the awake state and during anesthesia. In support of this, the activity deviations in trkB.DN mice manifested in both awake and anesthetized animals.

Analysis of the LFP oscillatory patterns, which provides a proxy for the cortical activity levels and overall synchrony, revealed further alterations. trkB.DN mice presented enhanced baseline LFP activity, and deficient induction of synchronous activity in response to social stimuli, affecting a (very) broad frequency band, including the $\gamma$ band. This deviates from what is observed in mice with global ablation of trkB in PV neurons during adolescence, which leads to reduced spontaneous LFP oscillations in frequencies $>10 \mathrm{~Hz}$ in combination with reduced sensory-evoked $\gamma$ activity in cortex (Xenos et al., 2018). The broadband alterations (increased baseline and decreased modulation) in the trkB.DN mice replicate findings in numerous studies of PV interneuron dysfunction (del Pino et al., 2013; Cho et al., 2015; Sohal and Rubenstein, 2019; Guyon et al., 2021) and decreased excitability of PV and/or impaired spike timing could be common denominators, as suggested by modeling (Bartos et al., 2007; Carlén et al., 2012; Jadi et al., 2016; and see further below).

\section{$\mathrm{BDNF} /$ trkB signaling and prefrontal $\mathrm{PV}$ interneurons in social behavior}

The trkB.DN mice responded with increased aggression toward a juvenile intruder, and our PV-specific genetic manipulation adds to a growing body of work coupling prefrontal BDNF/trkB signaling to social cognition and aggression (Ilchibaeva et al., 2018; Mikics et al., 2018). Mice with postnatal knock-out of trkB in a majority of corticolimbic GABAergic interneurons display disrupted formation of social hierarchies, with elevated social dominance and aggression in male mice (Tan et al., 2018). Importantly, in vitro experiments demonstrated increased spontaneous excitatory synaptic transmission in the mPFC, and optogenetic silencing of the PNs within the prelimbic cortex rescued the behavioral phenotype, coupling deficits in social behavior to trkB signaling and decreased cortical inhibition (Tan et al., 2018). Innovative optogenetic experiments in behaving mice have causally linked enhanced MPFC firing with impaired information transmission and social function (Yizhar et al., 2011). Of relevance, the deficits in social behavior could be ameliorated by optogenetic activation of mPFC PV interneurons. Our study provides insight into how $\mathrm{mPFC} \mathrm{PV}$ interneurons regulate excitatory $\mathrm{mPFC}$ activities during SI, and how the population dynamics underlying SI rely on the integrity of this neuronal subpopulation. It would be highly relevant to in future studies extend the investigation to other cognitive behaviors with relevance to the proposed link between altered prefrontal BDNF/ trkB signaling, PV inhibition, and etiology/symptomatology in psychiatric disorders (Hashimoto et al., 2005; Galloway et al., 2008; Andero et al., 2014; Notaras and van den Buuse, 2020).

\section{BDNF/trkB signaling in adult mPFC PV interneurons}

Our experiments show that reduced $\mathrm{BDNF} / \mathrm{trkB}$ signaling changes the intrinsic properties of mature PV interneurons. Notably, several measurements indicated decreased responsiveness of PV interneurons expressing trkB.DN, including a significantly increased membrane time constant. Further, under increased drive, PV interneurons expressing trkB.DN displayed a significantly increased firing rate adaptation, i.e., could not maintain the firing rate. The deviant firing rate adaptation suggests that impairments in inhibition could manifest rapidly at the network level, as observed in our in vivo experiment. The increased membrane time constant and the increased firing rate adaptation could reduce the sensitivity of PV interneurons to fast frequency inputs and lead to imprecise spike timing of PV interneurons, and plausibly affect network oscillatory activities involving PV interneurons (Otte et al., 2010; Wang, 2010; Jadi et al., 2016). In a very recent study, a novel optically activatable $\operatorname{trkB}$ was used for local and fast induction of $\operatorname{trkB}$ signaling in PV interneurons of the adult visual cortex (Winkel et al., 2020); 30-60 min after short-term optical trkB activation, the excitability of PV interneurons was significantly decreased (through effects on 
Kv.3 channels). Thus, it appears that both increased and decreased trkB activity can affect the excitability of PV interneurons through multiple mechanisms at different time scales. Also, in models with postnatal genetic knock-out of trkB, the responsiveness of PV interneurons is decreased (Tan et al., 2018). Compensatory adaptations are likely to be employed in response to long-term manipulations. However, our reduction of $\mathrm{BDNF} / \mathrm{trkB}$ signaling in mature PV interneurons rendered distinct morphologic changes not seen in the postnatal knock-out models. Postnatal knock-out of trkB in PV neurons decreases the number of neurites and their length and arborization (Zheng et al., 2011; Tan et al., 2018; Xenos et al., 2018). We found increased neurite complexity of $\mathrm{PV}$ interneurons expressing trkB.DN, affecting both axons and dendrites. Specifically, all investigated dendritic measurements were significantly increased in PV interneurons expressing trkB.DN, including the number and total length of the dendrites, as well as their complexity and ending radius. The axons were found to display particularly increased arborization at distances from the soma where the complexity of PV interneurons overall is highest. This is also the location where PV basket neurons densely innervate local PNs (Tremblay et al., 2016). It is difficult to reconcile to which degree the modifications represent adaptations aimed to compensate for the decreased responsiveness in the PV interneurons and the increased excitatory activity in the local network versus being a direct result of the reduced BDNF/ trkB signaling. The viral construct employed in the current study can be used for further investigations of short-term and long-term alteration of BDNF/trkB signaling in cell-types and brain regions of interest and should aid clarification of cell-autonomous trkB actions and compensatory adaptations. Further elucidation of how BDNF through interaction with a complement of receptor isoforms in different cell-types and subcellular compartments can instruct changes in morphologies and intrinsic properties is essential to forward our understanding of how adult circuit functions are regulated by neurotrophic action.

\section{References}

Agetsuma M, Hamm JP, Tao K, Fujisawa S, Yuste R (2018) Parvalbuminpositive interneurons regulate neuronal ensembles in visual cortex. Cereb Cortex 28:1831-1845.

Andero R, Choi DC, Ressler KJ (2014) BDNF-TrkB receptor regulation of distributed adult neural plasticity, memory formation, and psychiatric disorders. Prog Mol Biol Transl Sci 122:169-192.

Ardid S, Vinck M, Kaping D, Marquez S, Everling S, Womelsdorf T (2015) Mapping of functionally characterized cell classes onto canonical circuit operations in primate prefrontal cortex. J Neurosci 35:2975-2991.

Bakker R, Tiesinga P, Kötter R (2015) The scalable brain atlas: instant web-based access to public brain atlases and related content. Neuroinformatics 13:353366.

Barfield ET, Gourley SL (2018) Prefrontal cortical trkB, glucocorticoids, and their interactions in stress and developmental contexts. Neurosci Biobehav Rev 95:535-558.

Bartos M, Vida I, Jonas P (2007) Synaptic mechanisms of synchronized gamma oscillations in inhibitory interneuron networks. Nat Rev Neurosci 8:45-56.

Berg S, Kutra D, Kroeger T, Straehle CN, Kausler BX, Haubold C, Schiegg M, Ales J, Beier T, Rudy M, Eren K, Cervantes JI, Xu B, Beuttenmueller F, Wolny A, Zhang C, Koethe U, Hamprecht FA, Kreshuk A (2019) ilastik: interactive machine learning for (bio)image analysis. Nat Methods 16:1226-1232.

Butler A, Hoffman P, Smibert P, Papalexi E, Satija R (2018) Integrating single-cell transcriptomic data across different conditions, technologies, and species. Nat Biotechnol 36:411-420.

Buzsáki G, Wang XJ (2012) Mechanisms of gamma oscillations. Annu Rev Neurosci 35:203-225.

Cardin JA, Carlén M, Meletis K, Knoblich U, Zhang F, Deisseroth K, Tsai LH, Moore CI (2009) Driving fast-spiking cells induces gamma rhythm and controls sensory responses. Nature 459:663-667.
Carlén M, Meletis K, Siegle JH, Cardin JA, Futai K, Vierling-Claassen D, Rühlmann C, Jones SR, Deisseroth K, Sheng M, Moore CI, Tsai LH (2012) A critical role for NMDA receptors in parvalbumin interneurons for gamma rhythm induction and behavior. Mol Psychiatry 17:537-548.

Cellerino A, Maffei L, Domenici L (1996) The distribution of brain-derived neurotrophic factor and its receptor trkB in parvalbumin-containing neurons of the rat visual cortex. Eur J Neurosci 8:1190-1197.

Chao M V (2003) Neurotrophins and their receptors: a convergence point for many signalling pathways. Nat Rev Neurosci 4:299-309.

Cho KKA, Hoch R, Lee AT, Patel T, Rubenstein JLR, Sohal VS (2015) Gamma rhythms link prefrontal interneuron dysfunction with cognitive inflexibility in Dlx5/6+/ - mice. Neuron 85:1332-1343.

del Pino I, García-Frigola C, Dehorter N, Brotons-Mas JR, Alvarez-Salvado E, Martínez de Lagrán M, Ciceri G, Gabaldón MV, Moratal D, Dierssen M, Canals S, Marín O, Rico B (2013) Erbb4 deletion from fast-spiking interneurons causes schizophrenia-like phenotypes. Neuron 79:11521168.

Eide FF, Vining ER, Eide BL, Zang K, Wang XY, Reichardt LF (1996) Naturally occurring truncated trkB receptors have dominant inhibitory effects on brain-derived neurotrophic factor signaling. J Neurosci 16:3123-3129.

Fenner BM (2012) Truncated TrkB: beyond a dominant negative receptor. Cytokine Growth Factor Rev 23:15-24.

Ferreira TA, Blackman AV, Oyrer J, Jayabal S, Chung AJ, Watt AJ, Sjöström PJ, Van Meyel DJ (2014) Neuronal morphometry directly from bitmap images. Nat Methods 11:982-984.

Galloway EM, Woo NH, Lu B (2008) Persistent neural activity in the prefrontal cortex: a mechanism by which BDNF regulates working memory? Prog Brain Res 169:251-266.

Gorba T, Wahle P (1999) Expression of TrkB and TrkC but not BDNF mRNA in neurochemically identified interneurons in rat visual cortex in vivo and in organotypic cultures. Eur J Neurosci 11:1179-1190.

Guyon N, Zacharias LR, Oliveira ED, Kim H, Leite P, Lopes-aguiar C, Carlén M (2021) Network asynchrony underlying increased broadband gamma power. J Neurosci. Advance online publication. Retrieved Feb 16, 2021. doi: 10.1523/JNEUROSCI.2250-20.2021.

Hamm JP, Peterka DS, Gogos JA, Yuste R (2017) Altered cortical ensembles in mouse models of schizophrenia. Neuron 94:153-167.e8.

Hashimoto T, Bergen SE, Nguyen QL, Xu B, Monteggia LM, Pierri JN, Sun Z, Sampson AR, Lewis DA (2005) Relationship of brain-derived neurotrophic factor and its receptor TrkB to altered inhibitory prefrontal circuitry in schizophrenia. J Neurosci 25:372-383.

Holtmaat A, Caroni P (2016) Functional and structural underpinnings of neuronal assembly formation in learning. Nat Neurosci 19:1553-1562.

Hu H, Gan J, Jonas P (2014) Fast-spiking, parvalbumin + GABAergic interneurons: from cellular design to microcircuit function. Science 345:1255263.

Huang ZJ, Kirkwood A, Pizzorusso T, Porciatti V, Morales B, Bear MF, Maffei L, Tonegawa S (1999) BDNF regulates the maturation of inhibition and the critical period of plasticity in mouse visual cortex. Cell 98:739-755.

Ilchibaeva TV, Tsybko AS, Kozhemyakina RV, Kondaurova EM, Popova NK, Naumenko VS (2018) Genetically defined fear-induced aggression: focus on BDNF and its receptors. Behav Brain Res 343:102-110.

Jadi MP, Behrens MM, Sejnowski TJ (2016) Abnormal gamma oscillations in $\mathrm{N}$-methyl-D-aspartate receptor hypofunction models of schizophrenia. Biol Psychiatry 79:716-726.

Jiao Y, Zhang Z, Zhang C, Wang X, Sakata K, Lu B, Sun Q-Q (2011) A key mechanism underlying sensory experience-dependent maturation of neocortical GABAergic circuits in vivo. Proc Natl Acad Sci USA 108:12131-12136.

Kawaguchi Y, Kubota Y (1997) GABAergic cell subtypes and their synaptic connections in rat frontal cortex. Cereb Cortex 7:476-486.

Kowiański P, Lietzau G, Czuba E, Waśkow M, Steliga A, Moryś J (2018) BDNF: a key factor with multipotent impact on brain signaling and synaptic plasticity. Cell Mol Neurobiol 38:579-593.

Lee SE, Lee Y, Lee GH (2019) The regulation of glutamic acid decarboxylases in GABA neurotransmission in the brain. Arch Pharm Res 42:10311039.

Levy DR, Tamir T, Kaufman M, Parabucki A, Weissbrod A, Schneidman E, Yizhar O (2019) Dynamics of social representation in the mouse prefrontal cortex. Nat Neurosci 22:2013-2022. 
Longair MH, Baker DA, Armstrong JD (2011) Simple neurite tracer: open source software for reconstruction, visualization and analysis of neuronal processes. Bioinformatics 27:2453-2454.

Mantz J, Milla C, Glowinski J, Thierry AM (1988) Differential effects of ascending neurons containing dopamine and noradrenaline in the control of spontaneous activity and of evoked responses in the rat prefrontal cortex. Neuroscience 27:517-526.

Märtin A, Calvigioni D, Tzortzi O, Fuzik J, Wärnberg E, Meletis K (2019) A spatiomolecular map of the striatum. Cell Rep 29:4320-4333.e5.

Massi L, Lagler M, Hartwich K, Borhegyi Z, Somogyi P, Klausberger T (2012) Temporal dynamics of parvalbumin-expressing axo-axonic and basket cells in the rat medial prefrontal cortex in vivo. J Neurosci 32:1649616502.

Mathis A, Mamidanna P, Cury KM, Abe T, Murthy VN, Mathis MW, Bethge M (2018) DeepLabCut: markerless pose estimation of user-defined body parts with deep learning. Nat Neurosci 21:1281-1289.

Mikics É, Guirado R, Umemori J, Tóth M, Biró L, Miskolczi C, Balázsfi D, Zelena D, Castrén E, Haller J, Karpova NN (2018) Social learning requires plasticity enhanced by fluoxetine through prefrontal Bdnf-TrkB signaling to limit aggression induced by post-weaning social isolation. Neuropsychopharmacology 43:235-245.

Notaras M, van den Buuse M (2020) Neurobiology of BDNF in fear memory, sensitivity to stress, and stress-related disorders. Mol Psychiatry 25:22512274.

Ohira K, Hayashi M (2009) A new aspect of the TrkB signaling pathway in neural plasticity. Curr Neuropharmacol 7:276-285.

O’Neill KM, Akum BF, Dhawan ST, Kwon M, Langhammer CG, Firestein BL (2015) Assessing effects on dendritic arborization using novel Sholl analyses. Front Cell Neurosci 9:285.

Öner M, Kocakoç ID (2017) JMASM 49: a compilation of some popular goodness of fit tests for normal distribution: their algorithms and MATLAB codes (MATLAB). J Mod App Stat Meth 16:547-575.

Otte S, Hasenstaub A, Callaway EM (2010) Cell type-specific control of neuronal responsiveness by gamma-band oscillatory inhibition. J Neurosci 30:2150-2159.

Park H, Poo MM (2013) Neurotrophin regulation of neural circuit development and function. Nat Rev Neurosci 14:7-23.

Pessoa L (2019) Neural dynamics of emotion and cognition: from trajectories to underlying neural geometry. Neural Netw 120:158-166.

Picelli S, Björklund ÅK, Faridani OR, Sagasser S, Winberg G, Sandberg R (2013) Smart-seq2 for sensitive full-length transcriptome profiling in single cells. Nat Methods 10:1096-1100.

Reh RK, Dias BG, Nelson CA, Kaufer D, Werker JF, Kolb B, Levine JD, Hensch TK (2020) Critical period regulation across multiple timescales. Proc Natl Acad Sci USA 117:23242-23251.

Rodgers RJ, Dalvi A (1997) Anxiety, defence and the elevated plus-maze. Neurosci Biobehav Rev 21:801-810.

Schindelin J, Arganda-Carreras I, Frise E, Kaynig V, Longair M, Pietzsch T, Preibisch S, Rueden C, Saalfeld S, Schmid B, Tinevez JY, White DJ, Hartenstein V, Eliceiri K, Tomancak P, Cardona A (2012) Fiji: an opensource platform for biological-image analysis. Nat Methods 9:676-682.

Schmitzer-Torbert N, Jackson J, Henze D, Harris K, Redish AD (2005) Quantitative measures of cluster quality for use in extracellular recordings. Neuroscience 131:1-11.
Shamash P, Carandini M, Harris K, Steinmetz N (2018) A tool for analyzing electrode tracks from slice histology. bioRxiv 447995.

Sohal VS, Rubenstein JLR (2019) Excitation-inhibition balance as a framework for investigating mechanisms in neuropsychiatric disorders. Mol Psychiatry 24:1248-1257.

Sohal VS, Zhang F, Yizhar O, Deisseroth K (2009) Parvalbumin neurons and gamma rhythms enhance cortical circuit performance. Nature 459:698702 .

Stoilov P, Castren E, Stamm S (2002) Analysis of the human TrkB gene genomic organization reveals novel TrkB isoforms, unusual gene length, and splicing mechanism. Biochem Biophys Res Commun 290:10541065.

Susaki EA, Tainaka K, Perrin D, Kishino F, Tawara T, Watanabe TM, Yokoyama C, Onoe H, Eguchi M, Yamaguchi S, Abe T, Kiyonari H, Shimizu Y, Miyawaki A, Yokota H, Ueda HR (2014) Whole-brain imaging with single-cell resolution using chemical cocktails and computational analysis. Cell 157:726-739.

Takesian AE, Hensch TK (2013) Balancing plasticity/stability across brain development. Prog Brain Res 207:3-34.

Tan S, Xiao Y, Yin HH, Chen AI, Wah T, Je HS, Soong TW, Je HS (2018) Postnatal TrkB ablation in corticolimbic interneurons induces social dominance in male mice. Proc Natl Acad Sci USA 115:E9909-E9915.

Tremblay R, Lee S, Rudy B (2016) GABAergic interneurons in the neocortex: from cellular properties to circuits. Neuron 91:260-292.

van Lunteren JA, Fuzik J (2020) Script for spike-frequency adaptation plot. Zenodo. Available at http://doi.org/10.5281/zenodo.4315154.

Voigts J, Siegle JH, Pritchett DL, Moore CI (2013) The flexDrive: an ultralight implant for optical control and highly parallel chronic recording of neuronal ensembles in freely moving mice. Front Syst Neurosci 7:8.

Wang XJ (2010) Neurophysiological and computational principles of cortical rhythms in cognition. Physiol Rev 90:1195-1268.

Winkel F, Voigt MB, Didio G, Matéo S, Jetsonen E, Pou ML, Steinzeig A, Ryazantseva M, Harkki J, Englund J, Khirug S, Rivera C, Palva S, Taira T, Lauri SE, Umemori J, Castrén E (2020) Optical TrkB activation in Parvalbumin interneurons regulates intrinsic states to orchestrate cortical plasticity. bioRxiv 2020.04.27.063503.

Winslow JT (2003) Mouse social recognition and preference. Curr Protoc Neurosci Chapter 8:Unit 8.16.

Xenos D, Kamceva M, Tomasi S, Cardin JA, Schwartz ML, Vaccarino FM (2018) Loss of TrkB signaling in parvalbumin-expressing basket cells results in network activity disruption and abnormal behavior. Cereb Cortex 28:3399-3413.

Yeung MSY, Zdunek S, Bergmann O, Bernard S, Salehpour M, Alkass K, Perl S, Tisdale J, Possnert G, Brundin L, Druid H, Frisén J (2014) Dynamics of oligodendrocyte generation and myelination in the human brain. Cell 159:766-774.

Yizhar O, Fenno LE, Prigge M, Schneider F, Davidson TJ, O'Shea DJ, Sohal VS, Goshen I, Finkelstein J, Paz JT, Stehfest K, Fudim R, Ramakrishnan C, Huguenard JR, Hegemann P, Deisseroth K (2011) Neocortical excitation/inhibition balance in information processing and social dysfunction. Nature 477:171-178.

Zheng K, An JJ, Yang F, Xu W, Xu ZQD, Wu J, Hökfelt TGM, Fisahn A, Xu $\mathrm{B}$, Lu B (2011) TrkB signaling in parvalbumin-positive interneurons is critical for gamma-band network synchronization in hippocampus. Proc Natl Acad Sci USA 108:17201-17206. 